\title{
Structure and function evolution of thiolate monolayers on gold
}

\author{
by
}

\section{Grant Alvin Edwards}

\author{
A dissertation submitted to the graduate faculty \\ in partial fulfillment of the requirements for the degree of \\ DOCTOR OF PHILOSOPHY
}

Major: Analytical Chemistry

(Chemical Instrumentation and Materials Chemistry)

Program of Study Committee:

Marc D. Porter, Major Professor

Robert S. Houk

L. Scott Chumbley

Patricia A. Thiel

Andrew C. Hillier

Iowa State University

Ames, Iowa

2006

Copyright (C) Grant Alvin Edwards, 2006. All rights reserved. 


\section{Graduate College \\ Iowa State University}

This is to certify that the doctoral dissertation of

Grant Alvin Edwards

has met the dissertation requirements of Iowa State University

Major Professor

For the Major Program 


\section{DEDICATION}

In loving memory, Grant A. Edwards dedicates this thesis in the memory of Ronald L. Edwards, taken from us on November 4, 2005. He will always be remembered as a loving father and a wonderful friend. 


\section{TABLE OF CONTENTS}

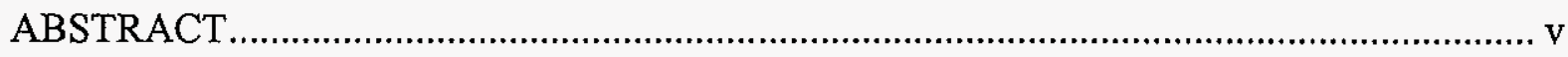

CHAPTER 1: GENERAL INTRODUCTION ..................................................................... 1

CHAPTER 2: A SUPRISING ODD-EVEN OSCILLATION IN THE

HETEROGENEOUS ELECTRON-TRANSFER RATE OF A

SOLUTION-BASED REDOX COUPLE AT ALKANETHIOLATE

MONOLAYERS ON GOLD

CHAPTER 3: MOLECULAR MODELING OF $n$-ALKANETHIOLATE

MONOLAYERS ON GOLD: ADLAYER ORIENTATION EFFECTS

CHAPTER 4: MASS TRANSPORT EFFECTS ON THE FORMATION OF

SELF-ASSEMBLED MONOLAYERS.

CHAPTER 5: HIGH RESOLUTION MAPPING OF COMPOSITIONAL DIFFERENCES AT ELECTRODE INTERFACES BY ELECTRIC FORCE MICROSCOPY

CHAPTER 6: SUMMARY AND PROSPECTUS 109

ACKNOWLEDGEMENTS 


\begin{abstract}
The use of $n$-alkanethiolate self-assembled monolayers on gold has blossomed in the past few years. These systems have functioned as models for common interfaces. Thiolate monolayers are ideal because they are easily modified before or after deposition. The works contained within this dissertation include interfacial characterization (infrared reflection absorption spectroscopy, ellipsometry, contact angle, scanning probe microscopy, and heterogeneous electron-transfer kinetics) and various modeling scenarios. The results of these characterizations present ground-breaking insights into the structure, function, and reproducible preparation of these monolayers. Surprisingly, three interfacial properties (electron-transfer, contact angle, and ellipsometry) were discovered to depend directly on the odd-even character of the monolayer components. Molecular modeling was utilized to investigate adlayer orientation, and suggests that these effects are adlayer structure specific. Finally, the electric force microscopy and theoretical modeling investigations of monolayer samples are presented, which show that the film dielectric constant, thickness, and dipole moment directly affect image contrast. In addition, the prospects for utilization of this emerging technique are outlined.
\end{abstract}




\section{CHAPTER 1: GENERAL INTRODUCTION}

\section{Organization and Overview}

Many technologically relevant processes occur at the interface between two materials, whether at a solid/liquid or biolipid interface. The combination of self-assembled monolayers (SAMs), scanning probe microscopy, interfacial electrochemistry, and surface sensitive spectroscopic methods has advanced the chemical understanding of these interfaces. The work presented in this dissertation is a culmination of projects with the express purpose of extending insights into the structure and resulting properties of interfaces modified by thin organic films.

The introduction section (Chapter 1) presents a brief overview of the structure of the model system, $n$-alkanethiolates on gold $\left(\mathrm{X}-\left(\mathrm{CH}_{2}\right)_{n} \mathrm{~S}-\mathrm{Au}\right)$, and operational principles of atomic force microscopy, null ellipsometry, wettability, electrochemical measurements of heterogeneous electron-transfer kinetics, and infrared reflection absorption spectroscopy.

Chapter 2 is the first original research chapter which presents the discovery of an odd-even chain length dependence of the heterogeneous electron-transfer kinetics for solution-based redox couples. This finding has led to Chapters 3 and 4, which include experimental investigations of adlayer properties. Chapter 3 is a molecular modeling investigation of the thiolate adlayer system as pertinent to wettability and thickness. This chapter also describes the first theoretical evidence for oscillations in wettability and thickness with alkyl chain length, as well as the experimental observation of oscillation in both properties. Chapter 4 details investigations into the controlled mass transport of thiol molecules to the gold surface. It discusses the importance of the deposition conditions on the reproducibility of the adlayer structure and resulting interfacial properties of the system. A theoretical model is constructed that relates substrate/vessel geometry, deposition solution 
concentration, and thiol mass transport to the surface. The model is then utilized as a platform to assess the adlayer structure dependence on deposition parameters. Chapter 5 completes the research portion of the dissertation with an investigation into the theoretical basis for the contrast mechanism in electric force microscopy (EFM) of monolayer samples, using a system of benzyl mercaptan derived-adlayers.

The dissertation is concluded (Chapter 6) with a brief summary of the research presented and a discussion of the possible future directions for this work.

\section{$\underline{n \text {-Alkanethiolates on Gold }}$}

Use of self-assembled $n$-alkanethiolate monolayers on gold $\left(\mathrm{X}-\left(\mathrm{CH}_{2}\right)_{n} \mathrm{~S}-\mathrm{Au}\right)$ is widespread. ${ }^{1-8}$ There are many excellent examples of fundamental and application-based areas related to electroanalytical and surface chemistry. ${ }^{6,9,10}$ For example, alkanethiolate monolayers are often employed to block electrochemical access of solution-based molecules to the metal substrate. ${ }^{11} \mathrm{~A}$ well-defined thickness between the substrate surface and redox couple is provided by SAMs with electroactive tail groups ${ }^{1-19}$ in which the alkyl chain positions the redox center a well defined distance from the electrode. This approach enables studies of the long-range heterogeneous electron transfer pathway. SAMs are also useful as a facile means to place other functional groups at the surface, an approach heavily exploited in manipulation of the selectivity of chemical sensors. ${ }^{9,20}$ As such, cyclodextrins, calixarenes, and other molecules with size and shape specificity can be tethered to the surface via gold-sulfur chemistry. ${ }^{21,22}$ Moreover, monolayers of alkanethiolates have been utilized in biomimetics, ${ }^{20}$ nanotechnology, ${ }^{8}$ molecular electronics, ${ }^{20}$ and in studies of interfacial phenomenon. ${ }^{8}$

Despite their widespread exploitation, the structure and assembly mechanism of thiolate monolayers on gold is widely debated..$^{20,23} \mathrm{~A}$ partial consensus has been reached regarding the structure achieved as the adlayer becomes saturated and approaches 
equilibrium. However, variations in surface pretreatment procedures and deposition conditions have led to conflicting views of the evolution in monolayer structure and resulting interfacial electrode properties. We attribute these complications directly to the assembly mechanism and intermolecular forces directing the finalized structure.

The assembly mechanism of alkanethiols onto gold from the liquid phase ${ }^{20,23-32}$ and the vapor phase ${ }^{33}$ has been investigated using scanning tunneling microscopy (STM) and other techniques. The adsorption process is characterized by four distinct stages. ${ }^{24}$ The initial stage can be described as a two-dimensional lattice-gas, in that molecules on the sparsely covered surface are highly mobile. Stage 2 begins with nucleation of molecular islands in which the alkyl chain lies parallel to the plane of the surface. In this stage, the surface coverage of the adsorbate is only a fraction of full coverage. In Stage 3, the molecular axis of the alkyl chain begins to reorient toward the surface normal. As the adlayer components begin to reorient, additional molecules can adsorb, filling in the areas vacated by the alkyl chains. In the final stage, an annealing process proceeds over a longer time scale. This stage is comprised of gauche to trans confirmation transitions of the alkyl chains and the filling adsorbate vacancies. The adsorption and assembly process is driven by interactions (e.g., van der Waals forces) between neighboring adsorbates. These lateral interactions orient the molecules more perpendicular to the surface, allowing the coverage and thickness to increase to a limiting value. The combination of head-group binding and intermolecular forces leads to an equilibrium structure characterized by high packing density (formation of a full monolayer), molecular ordering (predominately trans zigzag conformation of sequences of the alkane chains), and overall chemical and mechanical stability.

Although $n$-alkanethiolate monolayers are capable of forming dense adlayers, many subtle variables, especially during the early stages of formation, ${ }^{34,35}$ can result in the variation of the microscopic film structure. These differences can significantly alter the behavior of the adlayers, particularly those that have not approached the equilibrium structure. 
Many studies have investigated the characteristics of this system using techniques including: diffraction, ${ }^{36-44}$ ellipsometry, ${ }^{1,3,45-49}$ temperature programmed desorption, ${ }^{4,46,47}$ wettability, ${ }^{1,46-53}$ X-ray photoelectron spectroscopy, ${ }^{4,45-49,51,54}$ infrared reflection absorption spectroscopy, ${ }^{1,3,4,45,47,48,55,56}$ surface Raman scattering, ${ }^{57,58}$ scanning tunneling and atomic force microscopy, ${ }^{3,43,59-67}$ electron spectroscopies, ${ }^{4}$ and electron microscopy. ${ }^{42,45,46,48,68} \mathrm{~A}$ result of these characterizations is a broadly accepted structural description of these adlayers as they approach equilibrium.

For the adlayer structure on $\mathrm{Au}(111)$, the sulfur atom binds to the gold resulting in a gold thiolate, ${ }^{51,69,70}$ with a partial charge of -0.2 residing on the sulfur. ${ }^{71}$ The thiolates are presumably bound in the threefold hollow sites, ${ }^{72-74}$ although this has not been experimentally verified. The intermolecular forces and headgroup binding drive the adlayer to form a $(\sqrt{3} \times \sqrt{3}) \mathrm{R} 30^{\circ}$ overlayer structure, ${ }^{36-38,53,59,63,68,75}$ potentially with an extended superstructure. ${ }^{10}$ At equilibrium, the polymethylene chains extend from the surface in a nearly all-trans configuration with a tilt $(\alpha)$ of $\sim 30^{\circ}$ and twist $(\beta)$ of $\sim 50^{\circ}, 3,7,47,48,55,76$ The definition of $\alpha$ and $\beta$ are presented schematically in Figure 1, where $\alpha$ is the tilt of the C-C chain backbone from the surface normal, and $\beta$ is the twist of the $\mathrm{C}-\mathrm{C}$ chain with respect to a plane established by the chain axis and the surface normal. ${ }^{7}$

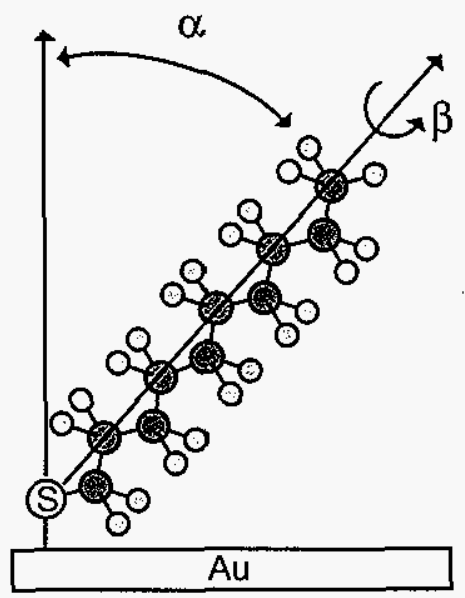

Figure 1. Schematic side view of an alkanethiolate $(n=9)$. The tilt $(\alpha)$ and twist $(\beta)$ angles are described in the text. 
Electrochemically, many of these adlayers are stable within an extended window of applied potential: -0.8 to $+0.8 \mathrm{~V}$ vs. $\mathrm{Ag} / \mathrm{AgCl},{ }^{75}$ although the identity of the tail group and the solution $\mathrm{pH}$ can alter these limits. Nevertheless, the positive limit is defined by oxidation of the adlayer, and electrochemical experiments have revealed that upon cathodic potential sweeps, a surface wave is observed that corresponds to the one-electron reduction of a complete monolayer of thiolates. ${ }^{77}$ The film packing density can be determined from the charge obtained upon reductive desorption. Moreover, reductive desorption has allowed insights into the adlayer formation mechanism. ${ }^{75}$

\section{Null Ellipsometry}

The use of light as a means to perform thickness measurements has been in force since before $1900{ }^{78}$ The technique has blossomed as a nondestructive tool for examination of thin films on a variety of substrates. As light is reflected by the sample, changes in the polarization state can be used to investigate some properties of the sample. Chapter 3 utilizes null ellipsometry to measure the thickness of alkanethiolate monolayers on gold, and therefore, this section is meant to convey a first principles understanding of the measurement.

The properties of the sample that are of particular interest are the complex refractive index $(N)$ and thickness $(d)$. The optical properties of any material are fully enveloped within $N$, which is a function of the optical constants, $n$ and $k$, as shown by Equation 1. Refractive

$$
N=n+i k
$$

processes are represented by $n$ (the refractive index) and absorption is described through $k$ (the extinction coefficient). Both the constituents of $N$ are wavelength and material dependent. In addition, the optical constants $n$ and $k$ are related by the Kramers-Kronig transformation. The complex refractive index of the material describes how light is affected by transmission and reflection. 


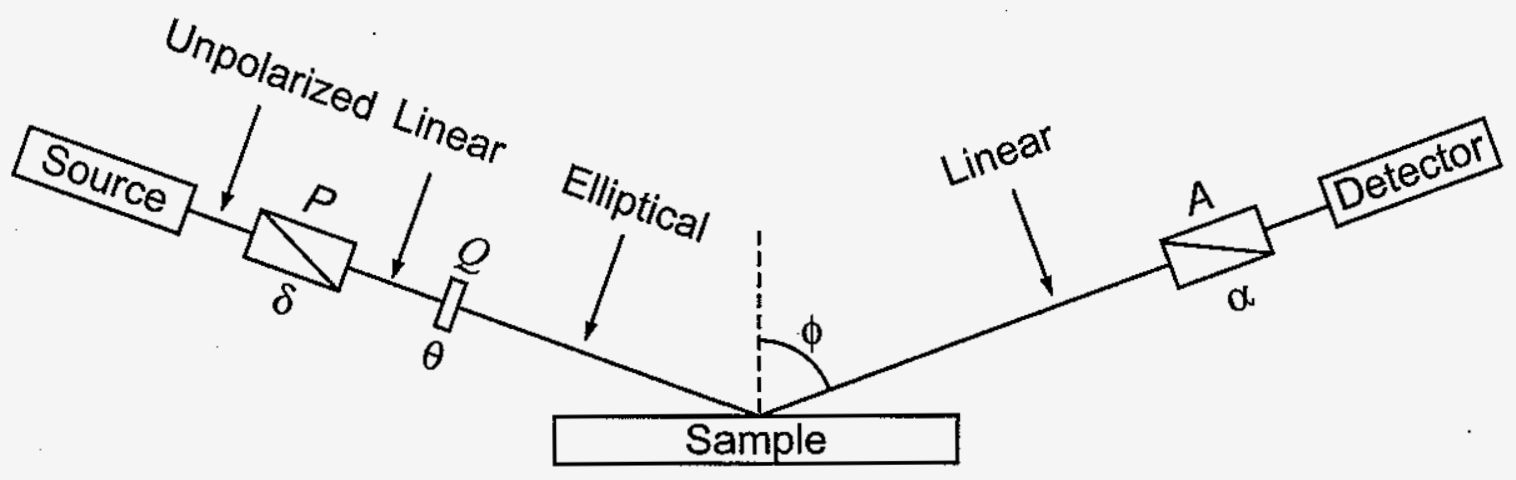

Figure 2. Configuration of a nulling monochromatic ellipsometer, showing the polarization state of the light after each optical element.

To this end, ellipsometry monitors how the polarization of light is reflected from the sample surface. Therefore, an ellipsometer is constructed such that the polarization of the light impinging the surface can be controlled, and that of the reflected light can be measured. A schematic of a typical instrument is included in Figure 2.

In the simplest experimental design (a null ellipsometer), a monochromatic light source is employed. The initially unpolarized source photons are transmitted through a linear polarizer $(P)$ which is able to rotate through various azimuthal angles $(\delta)$ as defined with respect to the plane of incidence. The next component in the optical path is a quarter-wave plate $(Q)$ (with an angle $\theta$, usually $\pm \pi / 4$ ), which introduces an elliptical polarization. The light then impinges the sample surface at a known angle of incidence $(\phi)$, where interactions with the sample surface alter its polarization state. Finally, the reflected light proceeds through a rotating analyzer $(A$, with angle $\alpha)$ and into a light-intensity sensitive detector.

For an ellipsometric measurement, the first step is to rotate the polarizer such that the photons reflected from the sample are linearly polarized. The reflected beam is then extinguished (nulled) by rotating the analyzer. The analyzer $(\alpha)$ and polarizer $(\delta)$ angles are utilized to calculate $\Delta$ and $\Psi$, the two fundamental ellipsometric parameters (Equations 2 and $3)^{78}$ 


$$
\Delta=2 \delta+\frac{\pi}{2}
$$

$\Psi=\alpha$

The next step uses a model system comprised of smooth, infinitely large, parallel slabs of material. In this way, the model takes the form of multilayered materials in which $N$ and $d$ for each layer vary. For instance, the parallel-layer, three-phase model most often employed for monolayer-coated samples is shown in Figure 3. The measured ellipsometric parameters are then used to iteratively fit values of $n_{\mathrm{f}}$ and $k_{\mathrm{f}}$ that satisfy the model.

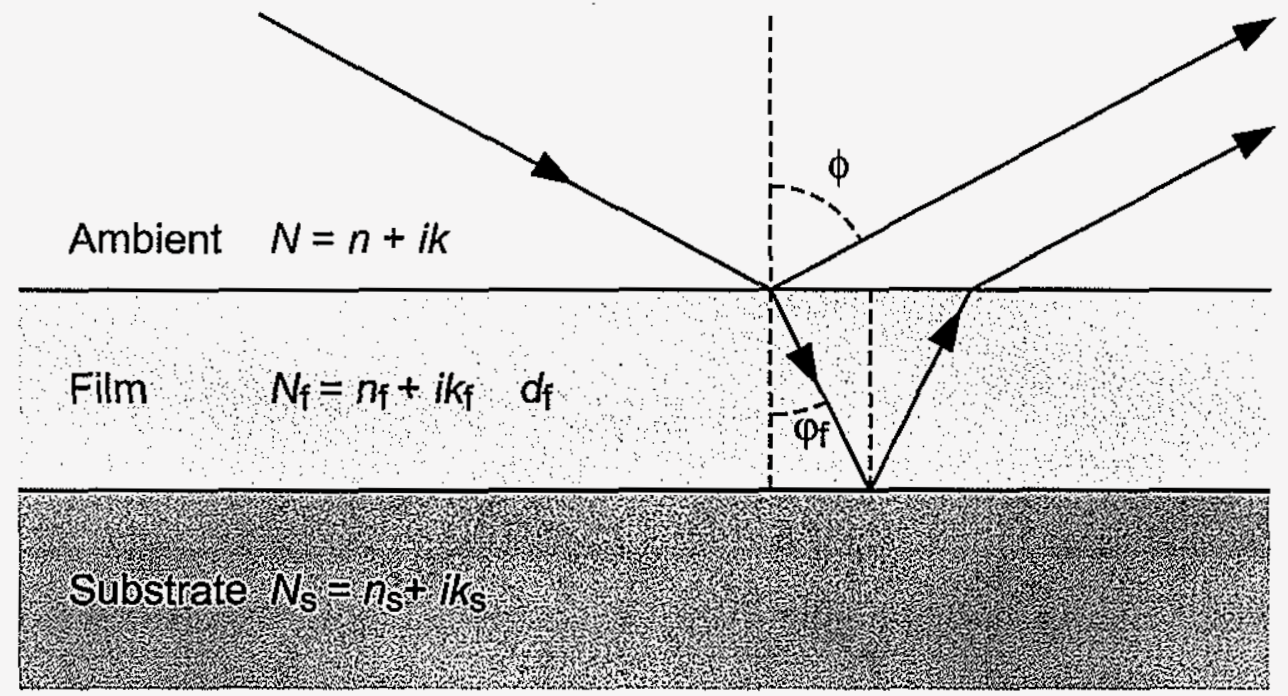

Figure 3. Parallel-layer, three-phase model utilized in calculation of substrate-film-ambient systems. $\varphi_{\mathrm{f}}$ is the angle of transmission of the light beam into the film.

Calculations within the iterative fit procedure focus on the reflection-induced change in polarization. The change is a result of the variation in reflection and transmission of the $s-$ and $p$-component polarizations (parallel and perpendicular to the sample surface, respectively) at each interface, which are described by the Fresnel coefficients. ${ }^{78}$ In addition, absorption of light is important if the incident wavelength overlaps an absorption band in the substrate or film. Mathematical treatment of the physical optics governing the interaction of the light with the sample has been recently reviewed by Tompkins, ${ }^{78}$ which includes a 
discussion of Fresnel reflection and transmission, Snell's law, and the resulting phase shift upon reflection for each polarization. Such a rigorous mathematical derivation is beyond the scope of this discussion. However, the ability to create and fit models to the experimental data is typically included within commercially available instrument software.

The use of the fit procedure also requires that the optical characteristics $\left(n_{\mathrm{s}}\right.$ and $\left.k_{\mathrm{s}}\right)$ of the underlying substrate be determined with high accuracy. In many cases, these constants introduce a significant source of error in calculation of the film thickness; therefore, reliable assessment is paramount. Frequently, these values are measured before deposition of the film, utilizing the fundamental ellipsometric constants, and a simplified substrate-ambient (two-phase) model.

\section{Heterogeneous Electron-Transfer Kinetics}

In Chapter 2, the heterogeneous electron-transfer rate constant $\left(k_{\text {app }}^{0}\right)$ between a solution-based redox molecule and a $n$-alkanethiolate monolayer-coated gold electrode is shown to exhibit an odd-even oscillation with respect to the monolayer chain length. The assessment of $k_{\text {app }}^{0}$ is accomplished by cyclic voltammetry (CV). Many monographs exist that discuss the benefits and drawbacks of this technique, ${ }^{11,79}$ and an introductory primer is presented herein.

For the purpose of this discussion, a theoretical redox reaction shown in Reaction 4 was chosen. It is assigned a formal reduction potential $\left(E^{0_{1}}\right)$ of $0 \mathrm{~V}$, a transfer coefficient $(\alpha)$ of 0.5 , and a diffusion coefficient $(D)$, which is equivalent for $\mathrm{O}$ and $\mathrm{R}$. In addition, the reactants and products participate in no side reaction (e.g., decomposition or coupled chemical/electrochemical reactions). A discussion of systems consisting of more complicated electron-transfer mechanisms have been presented elsewhere. ${ }^{11,79}$

$$
\mathrm{O}+\mathrm{ne}^{-} \stackrel{k_{2 p p}^{0}}{\longrightarrow} \mathrm{R}
$$


Additional assumptions are employed to simplify the solution conditions. ${ }^{79}$ At initiation of the experiments, the bulk solution contains only $\mathrm{R}$ and supporting electrolyte. Delivery of the electrochemically active molecules occurs in the semi-infinite linear diffusion regime. Conditions are employed such that the uncompensated solution resistance $\left(R_{\text {uncomp }}\right)$ is negligible. In addition, a background correction for the double-layer capacitance $\left(C_{\mathrm{dl}}\right)$ is assumed.

In $\mathrm{CV}$, the current flow to the working electrode is monitored while an applied potential $\left(E_{\text {appl}}\right)$ is used to drive faradaic and non-faradaic processes at the electrode surface. $E_{\text {appl }}$ is scanned linearly over a range of potentials (for example Figure $4 \mathrm{~A}$ ) from the initial potential $\left(E_{\text {initial }}\right)$ to a pre-defined limiting value $\left(E_{\text {switching }}\right)$ at a scan rate $(v)$. The direction of the potential scan is then reversed and the same potential window is scanned in the opposite direction. In this way, a species formed by oxidation on the forward scan may be reduced on the reverse scan. The resulting current-potential curve (voltammogram or CV) exhibits a shape diagnostic of the electron-transfer rate of the redox molecule and any side reactions.

The simplest voltammogram results for a reversible redox couple, in which the rate of conversion between $\mathrm{O}$ and $\mathrm{R}$ is fast relative to $\nu$. A representative voltammogram is shown in Figure 4B. For a reversible $k_{\text {app }}^{0}$, the concentration ratio of $\mathrm{O}$ to $\mathrm{R}([\mathrm{O}] /[\mathrm{R}])$ is dictated by the Nernst Equation (Equation 5); where $R$ is the ideal gas constant, $T$ is the absolute temperature, $\mathrm{n}$ is the electron stoichiometry, and $E^{01}$ is the formal reduction potential of the redox molecule. ${ }^{79}$ As long as the diffusion of molecules to the surface is sufficiently fast, the redox reaction at the surface with progress to the extent dictated by the Nernst Equation.

$$
E_{\text {appl }}=E^{0}+\frac{R T}{\mathrm{n} F} \ln \left(\frac{[\mathrm{O}]}{[\mathrm{R}]}\right)
$$


A

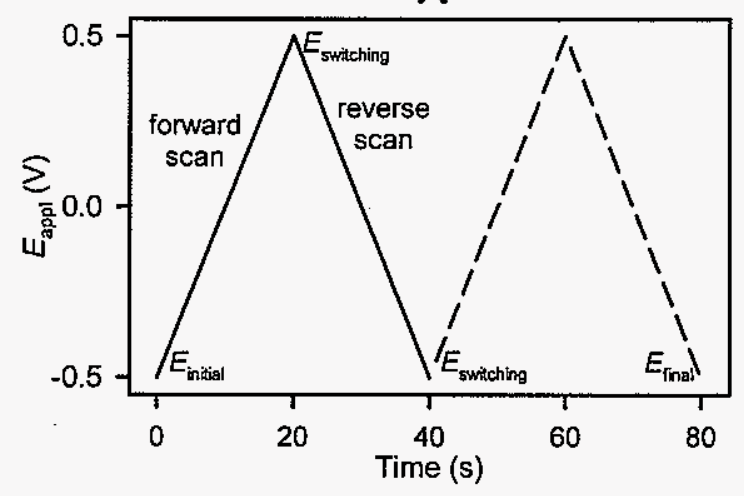

C

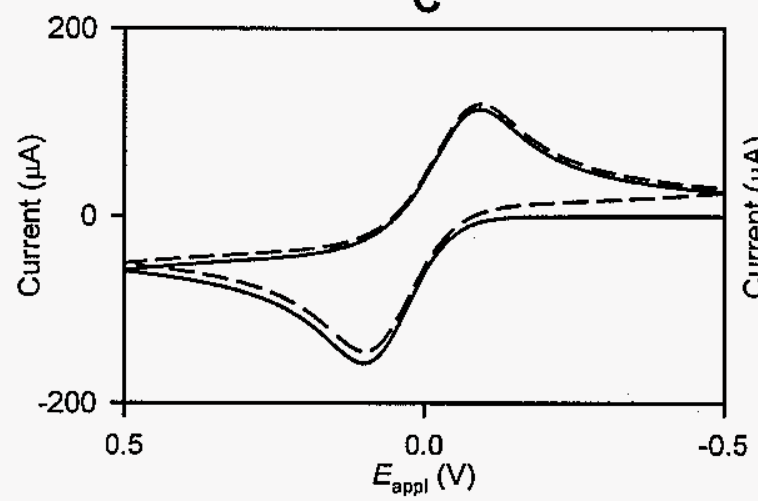

B

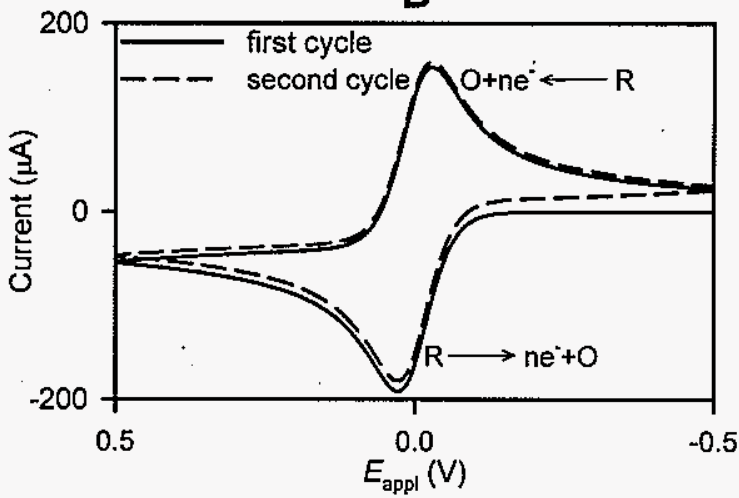

D

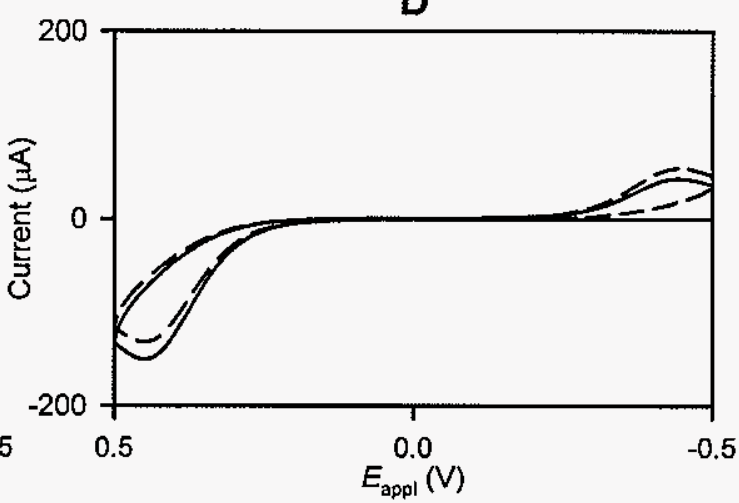

Figure 4. (A) Applied potential program for a two-cycle CV experiment. Example of resulting voltammograms simulated for with (B) a reversible $k_{\text {app }}^{0}$ $\left(2 \mathrm{~cm}^{2} \mathrm{~s}^{-1}\right),(\mathrm{C}) k^{0}$ app $=1 \times 10^{-3} \mathrm{~cm}^{2} \mathrm{~s}^{-1}$, and (D) $k_{\text {app }}^{0}=1 \times 10^{-6} \mathrm{~cm}^{2} \mathrm{~s}^{-1}$. CVs

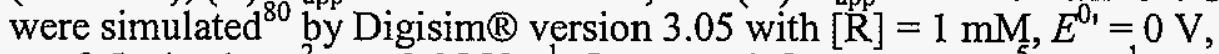
$\alpha=0.5, A=1 \mathrm{~cm}^{2}, \nu=0.05 \mathrm{~V} \mathrm{~s}^{-1}, R_{\text {uncomp }}=0 \Omega, D=1 \times 10^{-5} \mathrm{~cm} \mathrm{~s}^{-1}, \mathrm{n}=1$, and $C_{\mathrm{dl}}=10 \mu \mathrm{F} \mathrm{cm}^{-2}$.

At the initiation of the potential program, the required concentration ratio favors $R$, but as $E_{\text {appl }}$ sweeps more positive, the ratio begins to shift towards the oxidized form. In this way, electrons flow to the working electrode as the oxidation of $\mathrm{R}$ is driven by $E_{\text {appl. }}$. An increase in current is observed until a maximum value is reached. The peak occurs when the diffusion limited mass transfer of $\mathrm{R}$ to the surface is not sufficient to satisfy the concentration ratio requirement of the Nernst Equation; therefore, the concentration of $R$ at the surface becomes effectively zero at larger overpotential. At this point, the depletion of electroactive species near the electrode surface drives a decrease in current with time $(t)$, which follows a 


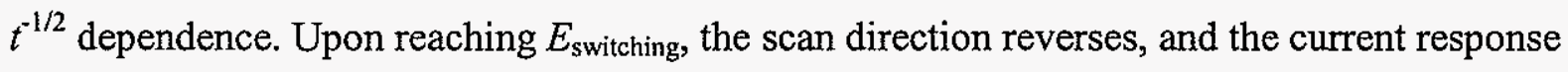
can be rationalized by again examining $[\mathrm{O}] /[\mathrm{R}]$ and the diffusion of molecules to the surface.

CVs exhibit characteristic shapes for reversible redox reactions. First, the anodic and cathodic peak positions are separated by $\sim 59 \mathrm{n}^{-1} \mathrm{mV}$. This peak separation $\left(\Delta E_{\mathrm{p}}\right)$ is invariant with scan rate (as long as $R_{\text {uncomp }}$ is small). In addition, the peak current is defined by the Randles-Sevcik equation (Equation 6) at $25^{\circ} \mathrm{C}$, where $i_{\mathrm{p}}$ is the peak current (A), $A$ is the electrode area $\left(\mathrm{cm}^{2}\right)$, and $D$ the diffusion coefficient $\left(\mathrm{cm}^{2} \mathrm{~s}^{-1}\right)$ and $C^{0}$ the bulk solution concentration (moles $\mathrm{cm}^{-3}$ ) of the active molecule. In this way, the peak current increases with $v^{1 / 2}$.

$$
i_{\mathrm{p}}=2.69 \times 10^{5} \mathrm{n}^{3 / 2} A D^{1 / 2} C^{0} v^{1 / 2}
$$

Voltammograms that do not exhibit the characteristics of a reproducible electron-transfer reaction discussed above are caused by slow electron exchange between the redox species and working electrode (i.e., a decrease in $k_{\text {app }}^{0}$ ). A moderate decrease in rate creates a quasireversible situation (Figure $4 \mathrm{C}$ ). $\mathrm{CVs}$ of this type are characterized by a $\Delta E_{\mathrm{p}}$ value between 60 and $212 \mathrm{mV}^{-1}$. A decrease in $k_{\text {app }}^{0}$ results in a larger peak separation because a higher overpotential is required before the redox reaction progresses fast enough to outpace diffusion. The peak separation is also dependent upon scan rate, with slower scans resulting is smaller $\Delta E_{\mathrm{p}}$ values. An estimate of $k_{\text {app }}^{0}$ can be found using Nicholson's method ${ }^{81}$ (Equation 7), where $f$ is $38.92 \mathrm{~V}^{-1}$. This method requires a dimensionless parameter, $\psi$, which is related to $\Delta E_{\mathrm{p}}{ }^{79,81,82}$

$$
k_{a p p}^{0}=\psi \sqrt{\pi D f v}
$$

As the reaction rate decreases further $\left(\Delta E_{\mathrm{p}}\right.$ increases larger than $\left.212 \mathrm{mV}\right)$, it enters the irreversible regime. Again, higher overpotentials are required to drive the redox reaction. An example of an irreversible voltammogram is shown in Figure 4D. Often the current profile for such an electrode reaction exhibits broad, poorly defined waves. This complication often 
necessitates a different way to measure $k_{\text {app }}^{0}$ that is not dependant upon peak position. The Butler-Volmer relationship ${ }^{79}$ (Equation 8, where $i$ is the faradaic current) fills such a void. This relationship allows calculation of the instantaneous reaction rate $(k)$ at any $E_{\text {appl }}$ based solely on the observed current. Translation to $k_{\text {app }}^{0}$ is straightforward, only requiring measurement of the faradaic current at $E^{0_{1}}$ after accounting for $C_{\mathrm{dl}}$.

$$
k=\frac{i}{\mathrm{nFAC}^{0}}
$$

\section{Wettability}

The use of contact angles as a facile means to interrogate interfacial tension has been widely used, especially for SAMs. ${ }^{1,46-52}$ The contact angle is obtained by placing a liquid drop on a flat solid surface and measurement of the angle at the liquid-solid-air interface. This angle is governed by the Young Equation (Equation 9), where the subscripts $s$ and $l$ denote solid and liquid respectively, $\theta$ is the contact angle, and $\gamma$ is the interfacial tension of the substance. This equation is derived by balancing the various interfacial forces acting upon the system, as presented in Figure $5 .{ }^{83}$

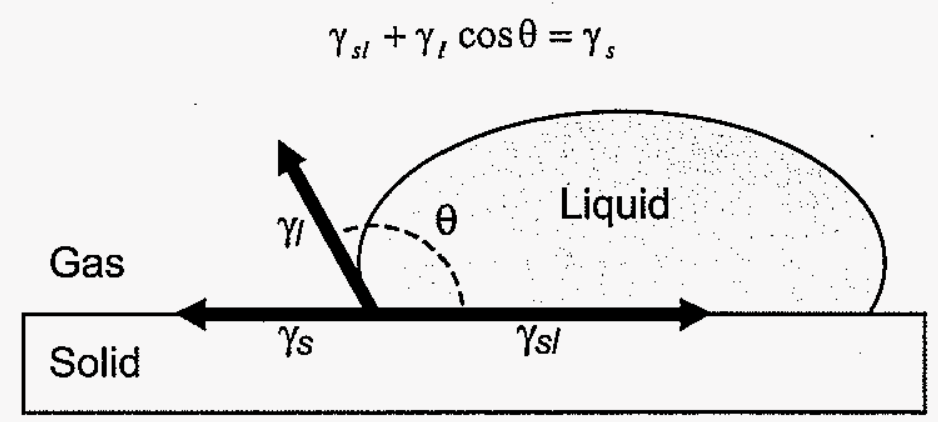

Figure 5. Diagram of surface forces active during a contact angle measurement.

Values of contact angle $(\theta)$ are very dependant upon the identity of the liquid and solid, as well as any chemical modification present on the surface or in the liquid. For instance, introduction of detergents into the liquid can modify the wetting of hydrophobic 
surfaces. In addition, contact angle hysteresis is the measurable difference between the advancing and receding values, as illustrated in Figure 6. The advancing contact angle $\left(\theta_{\mathrm{a}}\right)$ is measured after the liquid-solid-gas contact has spread across the surface. In contrast, the receding angle $\left(\theta_{\mathrm{r}}\right)$ is measured after the drop is partially withdrawn from the wetted surface. This hysteresis is most commonly attributed to chemical and/or physical heterogeneity of the surface (see Figure 15.5 in Israelachvili ${ }^{83}$ ). These heterogeneities cause metastable states at the solid-liquid-vapor interface and a departure from equilibrium defined by the Young Equation.

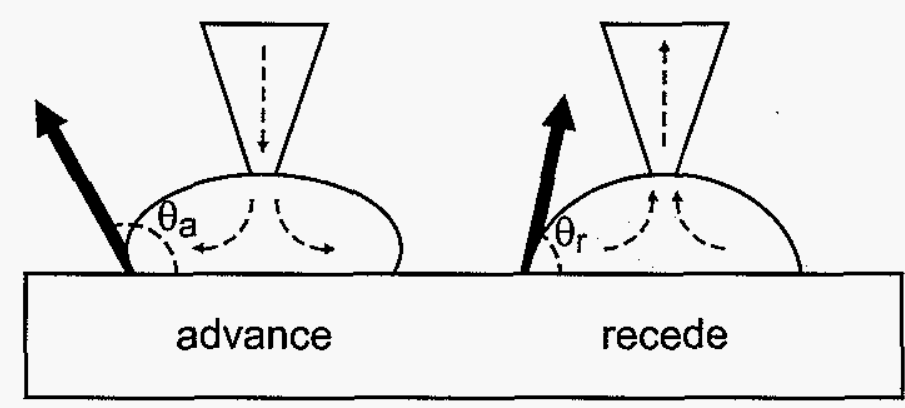

Figure 6. Schematic showing advancing $\left(\theta_{\mathrm{a}}\right)$ and receding $\left(\theta_{\mathrm{r}}\right)$ contact angles.

Extension of contact angle measurements for SAMs is straightforward, provided surface roughness and chemical homogeneity are controlled. Surface roughness is maintained by utilizing the same type of substrate for comparison between different monolayers. Within this dissertation, the substrate of choice is resistively evaporated gold on glass slides. It is assumed there is little batch-to-batch variability in surface roughness, and that the chemical homogeneity is controlled by the adlayer.

\section{Infrared Reflection Absorption Spectroscopy}

Infrared reflection absorption spectroscopy (IRRAS) has become an invaluable tool for characterization of surface phenomena for reasons including the multiplex advantage of Fourier transform interferometry and the development of high sensitivity, low-noise IR detectors. IRRAS has been heavily utilized to nondestructively interrogate the structure of 
thin films on reflective substrates, especially alkanethiolates on gold. ${ }^{1,3,4,45,47,48,55,56}$ Chapters 2 and 4 contain such IRRAS results, and therefore, the experimental requirements are discussed briefly here.

The instrumental diagram of a Fourier transform IR reflection instrument is shown in Figure 7. An IR beam is collimated and enters the interferometer. Upon leaving the interferometer, the beam then encounters a linear polarizer, which allows transmission of $p$-polarized light. The collimated beam impinges upon the sample surface at an incidence angle $(\phi)$, where it is reflected and focused into the $\mathbb{R}$ detector.

Reflection of the IR beam from the sample surface introduces optical effects that dramatically affect the resulting absorption spectra; therefore, the influence of the optical constants of the sample on the electric field at the surface must be understood. Variations in the absorption coefficient across an absorption band also affect the reflectivity of the interface. In other words, the intensity of the reflected light depends on the complex refractive index ( $N$, Equation 1$)$. In addition, the phase shift of the incoming photons upon reflection dictates which vibrational modes can be excited.

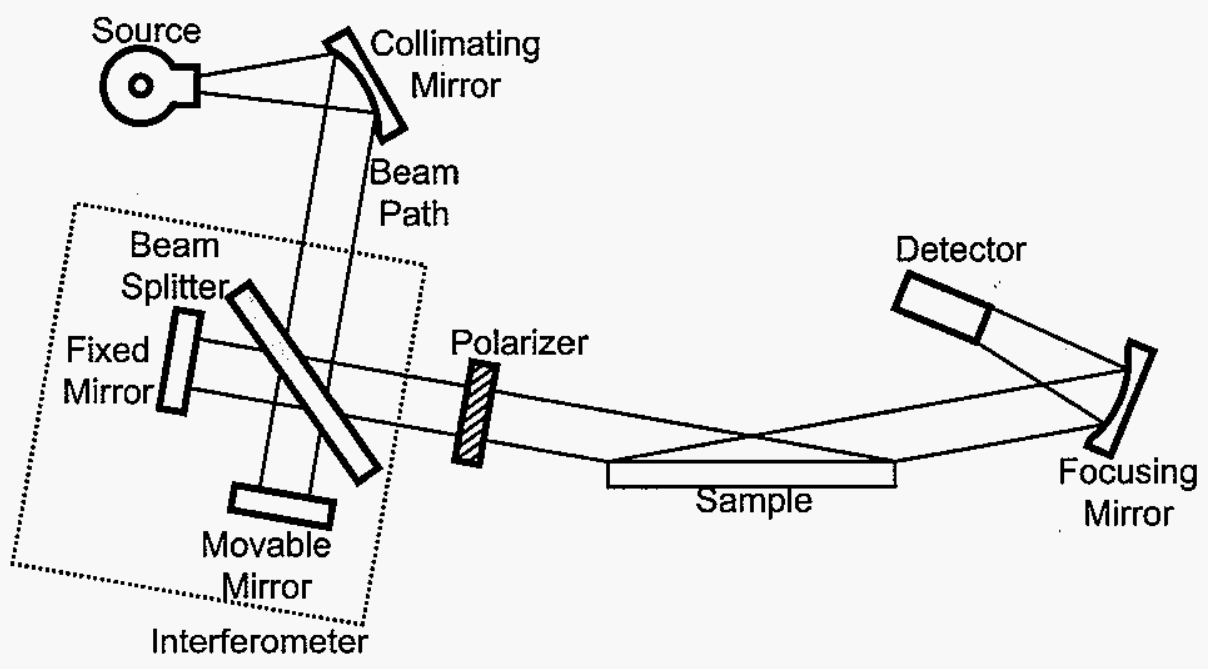

Figure 7. Schematic diagram of a typical IRRAS spectrometer. 
The model system, identical to that for ellipsometry, developed for investigation of these affects is shown in Figure 3. The book section by Lipert ${ }^{84}$ discusses the development of this model and an in-depth examination of the calculations; therefore, only the physical ramifications are discussed here. The model system still assumes the interfaces consist of semi-infinite planar, parallel optical boundaries, and a substrate of infinite thickness. ${ }^{84}$ The incident IR light is reflected or transmitted at each interface as dictated by $N$; however, larger contributions by absorption must be included.

In order to describe the absorbance and reflectance at these interfaces, a definition of the two component polarizations is required. Parallel-polarized light $(p)$ is polarized in the plane of incidence, parallel to the surface normal, and is comprised of $x$ - and $z$-components. The s-polarized light only has a $y$-component (parallel to the surface plane). These two polarization states interact with the surface in different ways and are described separately.

For s-polarized light that impinges on gold, a plot of the mean squared electrical field vs. distance from the electrode surface exhibits a periodic nature with a node at the metal surface (i.e., the electrical field is zero). ${ }^{84,85}$ The maximum in the field oscillation occurs at approximately one wavelength of the light from the sample surface. Therefore, if the sample film thickness is much less than this standing wave period, it will not interact with the $s$-polarized light. Therefore, the lack of electric field in the surface precludes the appearance in the spectrum of any vibrational mode whose change in dipole moment is parallel to the plane.

In contrast, the interaction of the $p$-polarized light creates a maximum net electrical field in the direction of the surface normal, with an approximately equal standing wave period. In fact, depending upon the angle of incidence the mean square electric field at the surface can be magnified up to four times that of the incoming field. ${ }^{84}$ This effect leads to an increased surface sensitivity for molecular vibrations parallel to the surface normal. The highest electrical field magnitude and therefore surface sensitivity occurs at a $\phi$ of $88^{\circ}$. 
However, experimental angles of closer to $80^{\circ}$ are frequently employed to facilitate reproducible sample-beam alignment.

The inequality in the electric field orientation serves as a basis for the loosely termed "infrared reflection surface selection rule." In this way, the absorbance intensity of vibrational modes in IRRAS spectroscopy is dictated by both the absorption cross-section and the transition dipole orientation with respect to the surface normal. This dependence is utilized to estimate the average orientation of thin films on metallic substrates. ${ }^{48,76,84}$ Therefore, the intensity of various absorption bands in the resulting spectra are used as a benchmark to estimate the alkyl chain orientation in Chapters 2 and 4.

\section{Atomic Force Microscopy}

Since its development by Binnig, Quate, and Gerber in $1986,{ }^{86}$ the atomic force microscope (AFM) has undergone a dramatic evolution in instrument design and usage. In its first 20 years of existence, the scanning modes of AFM have blossomed from a rudimentary contact mode into the dynamic modes of intermittent contact (TappingMode®) and noncontact mode. Tip and cantilever subassemblies are now commercially available, routinely manufactured using lithographic techniques from a variety of materials to achieve the resonance frequency, spring constant, and tip shape required for a particular experiment. In Chapter 5 the AFM was operated in TappingMode $B(T M)$ and the related electric force microscopy (EFM) mode, therefore, a short discussion of the instrumental caveats of these experimental setups is worthwhile.

\section{Tapping Mode}

In TM, the oscillating tip interacting with the sample is used to image surface topography and in some cases its mechanical properties. The tip is "tapping" at a high frequency on the sample surface, while its spatial coordinates are varied. The requirements of such a measurement are discussed here. 
The typical TM-AFM instrument (Figure 8) is comprised of a number of main components: a cantilever position-sensing device, a cantilever/tip assembly, a cantilever drive piezo, a sample or tip positioning piezo, feedback circuitry, and computer interface for data collection and experimental control. The components are fundamentally similar for each type of AFM, although various geometries have been developed.

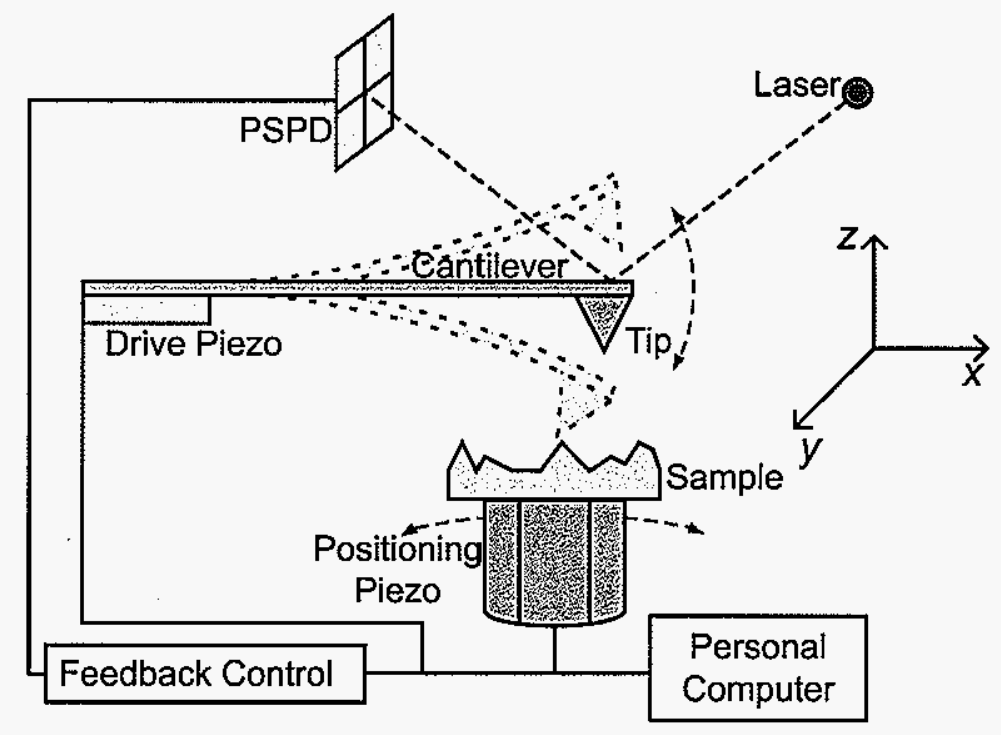

Figure 8. A schematic of a typical TM-AFM instrument.

The optical lever is only one of many different cantilever position sensing systems available, each with different characteristics and inherent noise; ${ }^{87}$ however, it is cost effective and widely implemented. In this method, the beam from a diode laser is reflected from the back of the cantilever near the tip, and into a position sensitive photodiode (PSPD). The fourquadrant diode is able to relay the vertical deflection of the cantilever to the feedback circuitry in real time.

The cantilever/tip assembly is comprised of an ultrasharp tip and at the end of the cantilever. The end of the tip interacts with the sample surface, and the response of the cantilever is used to image the surface. The dimensions and material of the assembly dictate the mechanical and chemical properties. They are available in different materials and 
geometries from various vendors, and can be purchased with a wide range of spring constants and geometries for specialized experiments. Typically, they are made of silicon with dimension of $4 \times 30 \times 125 \mu \mathrm{m}$, a resonance frequency about $300 \mathrm{kHz}$, a force constant of $\sim 75 \mathrm{~N} \mathrm{~m}^{-1}$, a tip height of $15 \mu \mathrm{m}$, and a tip radius of $\sim 10 \mathrm{~nm}$.

The two piezos (drive and positioning) are comprised of piezoelectric materials. This type of material responds to electric fields by extending or retracting along a crystallographic axis. The cantilever drive piezo is composed of layers of active material electrically controlled by the scan software. This allows the cantilever oscillation frequency and amplitude to be easily controlled within the software. The positioning piezo (scanner) is used to position the sample or tip in three dimensions. The scanner can be manufactured in a tube like geometry where movement in the $x$ - and $y$-directions is accomplished by bending of the tube, and is controlled within the software by a combination of scan size and rate. The $z$-direction position is enacted by extension or retraction of the scanner. This position is dictated by the feedback system attempting to counteract variations in the error signal as discussed below. The movement of the scanner is ultimately collected as the height image.

The feedback controller acts to unify the other components into a coherent instrument. In essence, the controller allows the positioning piezo to follow the height features of the sample. In TM, the amplitude of the oscillating cantilever is frequently used as the feedback mechanism. The user chooses a setpoint amplitude, which is then compared to the actual cantilever oscillation. The differences in these amplitudes comprise the error signal. Variations in sample topography cause the error signal to change, after which feedback directs the extension or retraction of the scanner. In this way, the feedback circuit allows the scanner to react quickly to changes in height. In addition, the feedback circuitry also allows measurement of the phase lag of the cantilever behind the piezo, which is collected as the phase image. 
Collectively, the imaging of sample topography and cantilever phase lag depend upon the dynamics of the interaction between the tip and sample surface. The quantitative analysis of the interactions is complicated by their characteristic nonlinear dynamics. Typically the system is modeled as a damped harmonic oscillator, with rigorous theoretical treatment of the oscillating cantilever system contained elsewhere. ${ }^{87,88}$ Fortunately, a qualitative understanding of imaging can be established without a mathematical description.

Initially, when the tip-sample separation is large, the drive piezo oscillates the cantilever at a specific amplitude and frequency. At this point, the sample surface has no effect on the oscillation amplitude. As the tip encounters the surface, the oscillation amplitude decreases and the phase lag of the cantilever changes as interactions between the tip and sample become important. These interactions can be conceptualized as additional damping applied to the harmonic oscillator. The decrease in amplitude is used to effectively sense the location of the surface. Therefore, the setpoint value is chosen such that it is smaller than the initial amplitude. In this way, the feedback controller strives to hold the cantilever at the setpoint amplitude by changing the $z$-piezo position. The phase lag of the cantilever is related to the energy loss of the cantilever, and is used to investigate material properties of the sample.

\section{Electric Force Microscopy}

The EFM mode is a technique that relies on normal TM imaging. In addition to the normal imaging, additional electrical interactions of the tip are monitored. This mode quantitates the electric field gradients between the tip and surface. In 2000, our laboratory published the first use of this mode for imaging compositional variations at buried interfaces ${ }^{89}$ and monitoring reactions at the same interface. ${ }^{90}$ These discoveries show that this technique will become a crucial tool in evaluating additional monolayer properties; however, widespread utilization requires the development of a theoretical model for the image contrast 
mechanism. A preliminary model is developed below after a brief description of EFM operating basics. Chapter 6 discusses the use of a system of benzyl mercaptans for comparison with the model developed herein.

Mechanistically, EFM imaging is operated in conjunction with TM. After scanning each line of the TM image, a second LiftMode $®$ pass retraces the same line at a constant height away from the surface $\left(z_{\text {tip }}\right)$, typically on the order of $100 \mathrm{~nm}$. This distance is chosen so that the tip is oscillating in a region where only electric forces are encountered. The physical properties of the tip can enhance electric field sensitivity. Therefore, tips with a high level of conductivity (whether inherent or artificially coated by a thin layer of conductive material) upon which a potential is applied are utilized to interrogate the electric field fluctuations across a sample.

The phase lag $(\Delta \Phi)$ of the cantilever is used to monitor the electric field variations. An image of $\Delta \Phi$ as a function of sample position can be related to the encountered electric field gradients. If the EFM tip-sample system can be modeled as a parallel plate capacitor with multiple dielectric layers as shown schematically in Figure 9, $\Delta \Phi$ can be related to the force gradient (Equation 10, where $Q=$ quality factor of the cantilever, $k=$ spring constant, and $F_{\text {elec }}$ is the electric force between the tip and sample) ${ }^{88}$ Equation 11 defines $F_{\text {elec }}$ for a parallel capacitor, where $C$ is the capacitance, $V=$ Voltage across the capacitor, and $z$ is the distance between the plates. ${ }^{87}$

$$
\begin{gathered}
\Delta \Phi=\frac{Q}{k} \frac{\partial F_{\text {elec }}}{\partial z} \\
F_{\text {elec }}=\frac{\delta C}{\delta z} \frac{\Delta V^{2}}{2}
\end{gathered}
$$

The EFM system can be modeled in this way (both with and without a polymer overlayer as discussed in previous reports), ${ }^{89,90}$ where the underlying gold substrate is grounded, a variable potential is applied to the tip, and the dielectric material consists of the 
adlayer itself. The model can be further discussed in various parts, modifying the effective capacitance and voltage of the system.

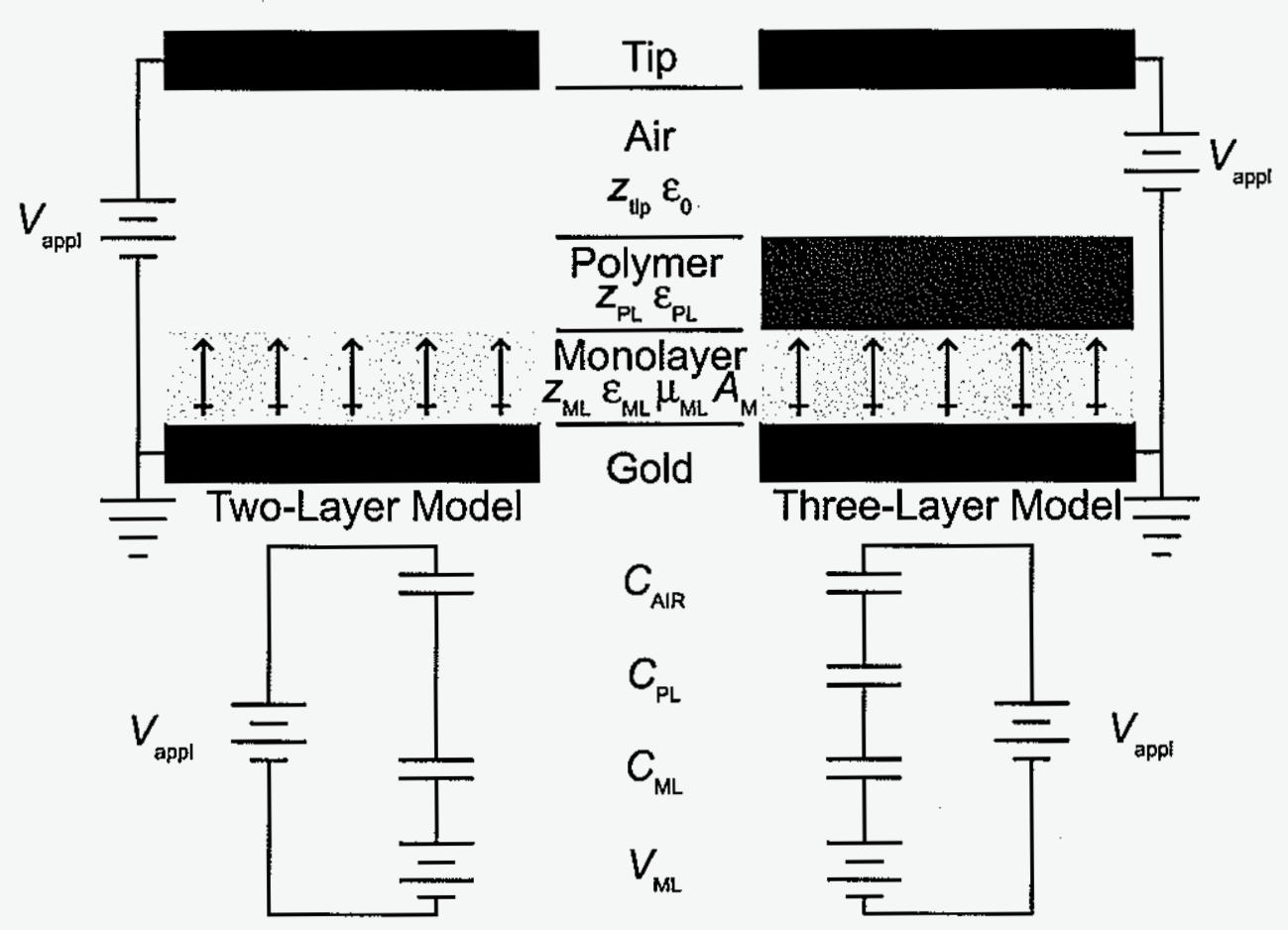

Figure 9. Model of the EFM imaging system, monolayers on gold with and without a polymer overlayer.

Both models contain the monolayer itself, with two properties of importance, both the surface potential created by the adlayer $\left(V_{\mathrm{ML}}\right)$ and its capacitance $\left(C_{\mathrm{ML}}\right)$. $V_{\mathrm{ML}}$ is dictated by Equation $12^{91}$ where $\mu_{\mathrm{ML}}$ is the dipole moment of the components in the direction of the surface normal, $A_{\mathrm{M}}$ is the molecular area of one molecule on the surface, $\varepsilon_{\mathrm{ML}}$ is their dielectric constant, and $\varepsilon_{0}$ is the permittivity of free space. $C_{\mathrm{ML}}$ is calculated by Equation 13, where $z_{\mathrm{ML}}$ is the thickness of the monolayer and $A_{\mathrm{c}}$ is the area of the monolayer interrogated by the EFM system. Furthermore, capacitive effects due to the volume between the tip and sample surface are dictated by Equation 14, a polymer layer, if present, by Equation 15 .

$$
V_{\mathrm{ML}}=\frac{\mu_{\mathrm{ML}}}{A_{\mathrm{M}} \varepsilon_{0} \varepsilon_{\mathrm{ML}}}
$$




$$
\begin{gathered}
C_{\mathrm{ML}}=\frac{A_{\mathrm{c}} \varepsilon_{0} \varepsilon_{\mathrm{ML}}}{z_{\mathrm{ML}}} \\
C_{\mathrm{AIR}}=\frac{A_{\mathrm{c}} \varepsilon_{0}}{z_{\text {tip }}} \\
C_{\mathrm{PL}}=\frac{A_{\mathrm{c}} \varepsilon_{0} \varepsilon_{\mathrm{PL}}}{z_{\mathrm{PL}}}
\end{gathered}
$$

Therefore, the total capacitance $\left(C_{\mathrm{tot}}\right)$ for the two model systems is given in either Equation 16 or 17, with and without the polymer layer respectively. The second differential of each capacitance is shown in Equations 18 and 19.

$$
\begin{gathered}
C_{\text {tot }}=\frac{A_{\mathrm{c}} \varepsilon_{0}}{\frac{z_{\mathrm{ML}}}{\varepsilon_{\mathrm{ML}}}+z_{\text {tip }}} \\
C_{\text {tot }}=\frac{A_{\mathrm{c}} \varepsilon_{0}}{\frac{z_{\mathrm{ML}}}{\varepsilon_{\mathrm{ML}}}+\frac{z_{\mathrm{PL}}}{\varepsilon_{\mathrm{PL}}}+z_{\mathrm{tip}}} \\
\frac{\partial^{2} C_{\mathrm{tot}}}{\partial z^{2}}=\frac{2 A_{\mathrm{c}} \varepsilon_{0}}{\left(\frac{z_{\mathrm{ML}}}{\varepsilon_{\mathrm{ML}}}+z_{\text {tip }}\right)^{3}} \\
\frac{\partial^{2} C_{\mathrm{tot}}}{\partial z^{2}}=\frac{2 A_{\mathrm{c}} \varepsilon_{0}}{\left(\frac{z_{\mathrm{ML}}}{\varepsilon_{\mathrm{ML}}}+\frac{z_{\mathrm{PL}}}{\varepsilon_{\mathrm{PL}}}+z_{\text {tip }}\right)^{3}}
\end{gathered}
$$

A combination of Equations $10,11,12,18$, and 19 result in Equations 20 and 21. These equations relate variations in sample parameters into an expected phase shift of the oscillating cantilever. This shift is highly dependent upon properties of the adlayer itself ( $z_{\mathrm{ML}}$, $\left.\varepsilon_{\mathrm{ML}}, A_{\mathrm{M}}, \mu_{\mathrm{ML}}\right)$ and imaging properties $\left(A_{\mathrm{c}}, z_{\mathrm{tip}}, Q, k\right)$. Not surprising is the inclusion of $\mathrm{A}_{\mathrm{c}}$, which is further dependant upon tip geometry and $z_{\text {tip }} .{ }^{92-94}$ The preliminary model developed herein does not include geometry parameters for the tip except within this parameter. 


$$
\begin{gathered}
\Delta \Phi=\frac{Q}{k} \frac{A_{\mathrm{c}} \varepsilon_{0}\left(V_{\text {tip }}-\frac{\mu_{\mathrm{ML}}}{A_{\mathrm{M}} \varepsilon_{0} \varepsilon_{\mathrm{ML}}}\right)^{2}}{\left(\frac{z_{\mathrm{ML}}}{\varepsilon_{\mathrm{ML}}}+z_{\text {tip }}\right)^{3}} \\
\Delta \Phi=\frac{Q}{k} \frac{A_{\mathrm{c}} \varepsilon_{0}\left(V_{\text {tip }}-\frac{\mu_{\mathrm{ML}}}{A_{\mathrm{M}} \varepsilon_{0} \varepsilon_{\mathrm{ML}}}\right)^{2}}{\left(\frac{z_{\mathrm{ML}}}{\varepsilon_{\mathrm{ML}}}+\frac{z_{\mathrm{PL}}}{\varepsilon_{\mathrm{PL}}}+z_{\text {tip }}\right)^{3}}
\end{gathered}
$$

The development of this model is one step in the development and proliferation of quantitative EFM imaging. Further model development will require inclusion of tip shape parameters and a theoretical examination of electrical field gradients during propagation through polymer films. Chapter 5 uses a system of benzyl mercaptan-derived adlayers for investigation of model validity.

\section{$\underline{\text { References }}$}

(1) Nuzzo, R. G.; Allara, D. L. J. Am. Chem. Soc. 1983, 105, 4481-3.

(2) Li, T. T.-T.; Weaver, M. J. J. Am. Chem. Soc. 1984, 106, 6107-8.

(3) Porter, M. D.; Bright, T. B.; Allara, D. L.; Chidsey, C. E. D. J. Am. Chem. Soc. 1987, 109, 3559-68.

(4) Nuzzo, R. G.; Zegarski, B. R.; Dubois, L. H. J. Am. Chem. Soc. 1987, 109, 733-40.

(5) Zhong, C.-J.; Porter, M. D. Anal. Chem. 1995, 67, 709A-15A.

(6) Ulman, A. An Introduction to Ultrathin Organic Films From Langmuir-Blodgett to Self-Assembly; Academic Press: San Diego, 1991.

(7) Dubois, L. H.; Nuzzo, R. G. Annu. Rev. Phys. Chem. 1992, 43, 437-63.

(8) Love, J. C.; Estroff, L. A.; Kriebel, J. K.; Nuzzo, R. G.; Whitesides, G. M. Chem. Rev. 2005, 105, 1103-69.

(9) Flink, S.; van Veggel, F. C. J. M.; Reinhoudt, D. N. Sensors Update 2001, 8, 3-19.

(10) Ulman, A. Chem. Rev. 1996, 96, 1533-54. 
(11) Finklea, H. O. "Electrochemistry of Organized Monolayers of Thiols and Related Molecules on Electrodes" In Electroanalytical Chemistry: A Series of Advances; Bard, A. J., Rubinstein, I., Eds.; Marcel Dekker, Inc.: New York, 1996; Vol. 19, p 109-335.

(12) Miller, C.; Cuendet, P.; Grätzel, M. J. Phys. Chem. 1991, 95, 877-86.

(13) Miller, C.; Grätzel, M. J. Phys. Chem. 1991, 95, 5225-33.

(14) Terrettaz, S.; Becka, A. M.; Traub, M. J.; Fettinger, J. C.; Miller, C. J. J. Phys. Chem. $1995,99,11216-24$.

(15) Becka, A. M.; Miller, C. J. J. Phys. Chem. 1992, 96, 2657-68.

(16) Becka, A. M.; Miller, C. J. J. Phys. Chem. 1993, 97, 6233-9.

(17) Smalley, J. F.; Feldberg, S. W.; Chidsey, C. E. D.; Linford, M. R.; Newton, M. D.; Liu, Y.-P. J. Phys. Chem. 1995, 99, 13141-9.

(18) Sumner, J. J.; Weber, K. S.; Hockett, L. A.; Creager, S. E. J. Phys. Chem. B 2000, $104,7449-54$.

(19) Weber, K.; Hockett, L.; Creager, S. J. Phys. Chem. B 1997, 101, 8286-91.

(20) Schreiber, F. J. Phys.: Condens. Matter 2004, 16, R881-R900.

(21) Davis, F.; Stirling, C. J. M. Langmuir 1996, 12, 5365-74.

(22) Weisser, M.; Nelles, G.; Wenz, G.; Mittler-Neher, S. Sens. Actuators, B 1997, 38-39, 58-67.

(23) Sandhyarani, N.; Pradeep, T. Int. Rev. Phys. Chem. 2003, 22, 221-62.

(24) Camillone, N., III Langmuir 2004, 20, 1199-206.

(25) Hahner, G.; Wöll, C.; Buck, M.; Grunze, M. Langmuir 1993, 9, 1955-8.

(26) Qu, D.; Morin, M. J. Electroanal. Chem. 2002, 524-525, 77-80.

(27) Zhong, C.-J.; Woods, N. T.; Dawson, G. B.; Porter, M. D. Electrochem. Commun. 1999, 1, 17-21.

(28) Subramanian, R.; Lakshminarayanan, V. Electrochim. Acta 2000, 45, 4501-9.

(29) Cohen-Atiya, M.; Mandler, D. J. Electroanal. Chem. 2003, 550-551, 267-76.

(30) Pan, W.; Durning, C. J.; Turro, N. J. Langmuir 1996, 12, 4469-73.

(31) Karpovich, D. S.; Blanchard, G. J. Langmuir 1994, 10, 3315-22.

(32) Bain, C. D.; Whitesides, G. M. J. Am. Chem. Soc. 1989, 111, 7164-75. 
(33) Poirier, G. E.; Pylant, E. D. Science 1996, 272, 1145-8.

(34) Byloos, M.; Al-Maznai, H.; Morin, M. J. Phys. Chem. B 2001, 105, 5900-5.

(35) Teuscher, J. H.; Yeager, L. J.; Yoo, H.; Chadwick, J. E.; Garrell, R. L. Faraday Discuss. 1997, 107, 399-416.

(36) Chidsey, C. E. D.; Liu, G.-Y.; Rowntree, P. A.; Scoles, G. J. Chem. Phys. 1989, 91, 4421-3.

(37) Chidsey, C. E. D.; Loiacono, D. N. Langmuir 1990, 6, 682-91.

(38) Camillone, N., III; Chidsey, C. E. D.; Liu, G.-Y.; Putvinski, T. M.; Scoles, G. J. Chem. Phys. 1991, 94, 8493-502.

(39) Camillone, N., III; Chidsey, C. E. D.; Liu, G.-Y.; Scoles, G. J. Chem. Phys. 1993, 98, 3503-11.

(40) Dubois, L. H.; Zegarski, B. R.; Nuzzo, R. G. J. Chem. Phys. 1993, 98, 678-88.

(41) Camillone, N., III; Chidsey, C. E. D.; Liu, G.-Y.; Scoles, G. J. Chem. Phys. 1993, 98, 4234-45.

(42) Fenter, P.; Eberhardt, A.; Eisenberger, P. Science 1994, 266, 1216-8.

(43) Camillone, N., III; Eisenberger, P.; Leung, T. Y. B.; Schwartz, P.; Scoles, G.; Poirier, G. E.; Tarlov, M. J. J. Chem. Phys. 1994, 101, 11031-6.

(44) Camillone, N., III; Leung, T. Y. B.; Schwartz, P.; Eisenberger, P.; Scoles, G. Langmuir 1996, 12, 2737-46.

(45) Nuzzo, R. G.; Fusco, F. A.; Allara, D. L. J. Am. Chem. Soc. 1987, 109, 2358-68.

(46) Bain, C. D.; Troughton, E. B.; Tao, Y.-T.; Evall, J.; Whitesides, G. M.; Nuzzo, R. G. J. Am. Chem. Soc. 1989, 111, 321-35.

(47) Nuzzo, R. G.; Dubois, L. H.; Allara, D. L. J. Am. Chem. Soc. 1990, 112, 558-69.

(48) Laibinis, P. E.; Whitesides, G. M.; Allara, D. L.; Tao, Y.-T.; Parikh, A. N.; Nuzzo, R. G. J. Am. Chem. Soc. 1991, 113, 7152-67.

(49) Biebuyck, H. A.; Bain, C. D.; Whitesides, G. M. Langmuir 1994, 10, 1825-31.

(50) Bain, C. D.; Whitesides, G. M. J. Am. Chem. Soc. 1988, 110, 5897-8.

(51) Bain, C. D.; Biebuyck, H. A.; Whitesides, G. M. Langmuir 1989, 5, 723-7.

(52) Whitesides, G. M.; Laibinis, P. E. Langmuir 1990, 6, 87-96.

(53) Dubois, L. H.; Zegarski, B. R.; Nuzzo, R. G. J. Am. Chem. Soc. 1990, 112, 570-9.

(54) Bain, C. D.; Whitesides, G. M. J. Am. Chem. Soc. 1988, 110, 6560-1. 
(55) Nuzzo, R. G.; Korenic, E. M.; Dubois, L. H. J. Chem. Phys. 1990, 93, 767-73.

(56) Truong, K. D.; Rowntree, P. A. J. Phys. Chem. 1996, 100, 19917-26.

(57) Bryant, M. A.; Pemberton, J. E. J. Am. Chem. Soc. 1991, 113, 8284-93.

(58) Bryant, M. A.; Pemberton, J. E. J. Am. Chem. Soc. 1991, 113, 3629-37.

(59) Widrig, C. A.; Alves, C. A.; Porter, M. D. J. Am. Chem. Soc. 1991, 113, 2805-10.

(60) Alves, C. A.; Smith, E. L.; Porter, M. D. J. Am. Chem. Soc. 1992, 114, 1222-7.

(61) McCarley, R. L.; Kim, Y.-T.; Bard, A. J. J. Phys. Chem. 1993, 97, 211-5.

(62) McCarley, R. L.; Dunaway, D. J.; Willicut, R. J. Langmuir 1993, 9, 2775-7.

(63) Liu, G.-Y.; Salmeron, M. B. Langmuir 1994, 10, 367-70.

(64) Poirier, G. E.; Tarlov, M. J.; Rushmeier, H. E. Langmuir 1994, 10, 3383-6.

(65) Schönenberger, C.; Jorritsma, J.; Sondag-Huethorst, J. A. M.; Fokkink, L. G. J. J. Phys. Chem. 1995, 99, 3259-71.

(66) McDermott, C. A.; McDermott, M. T.; Green, J.-B.; Porter, M. D. J. Phys. Chem. 1995, 99, 13257-67.

(67) Paradis, E.; Rowntree, P. J. Electroanal. Chem. 2003, 550-551, 175-85.

(68) Strong, L.; Whitesides, G. M. Langmuir 1988, 4, 546-58.

(69) Sellers, H.; Ulman, A.; Shnidman, Y.; Eilers, J. E. J. Am. Chem. Soc. 1993, 115, 9389-401.

(70) Zhang, H.; Chung, S.-W.; Mirkin, C. A. Nano Lett. 2003, 3, 43-5.

(71) Zhong, C.-J.; Brush, R. C.; Andregg, J.; Porter, M. D. Langmuir 1999, 15, 518-25.

(72) Beardmore, K. M.; Kress, J. D.; Grønbech-Jensen, N.; Bishop, A. P. Chem. Phys. Lett. 1998, 286, 40-5.

(73) Lay, M. D.; Varazo, K.; Stickney, J. T. Langmuir 2003, 19, 8416-27.

(74) Sun, L.; Crooks, R. M. Langmuir 1993, 9, 1951-4.

(75) Widrig, C. A.; Chung, C.; Porter, M. D. J. Electroanal. Chem. 1991, 310, 335-59.

(76) Parikh, A. N.; Allara, D. L. J. Chem. Phys. 1992, 96, 927-45.

(77) Walczak, M. M.; Popenoe, D. D.; Deinhammer, R. S.; Lamp, B. D.; Chung, C.; Porter, M. D. Langmuir 1991, 7, 2687-93. 
(78) Tompkins, H. G.; Irene, E. A. Handbook of Ellipsometry; William Andrew Pub.: Norwich, NY, 2005.

(79) Bard, A. J.; Faulkner, L. R. Electrochemical Methods: Fundamentals and Applications; 2nd ed; John Wiley \& Sons, Inc.: New York, 2001.

(80) Rudolph, M.; Reddy, D. P.; Feldberg, S. W. Anal. Chem. 1994, 66, 589A-600A.

(81) Nicholson, R. S. Anal. Chem. 1965, 37, 1351-5.

(82) Geiger, W. E. "Instructional Examples of Electrode Mechanisms of Transition Metal Complexes" In Laboratory Techniques in Electroanalytical Chemistry; 2nd ed.; Kissinger, P. T., Heineman, W. R., Eds.; Marcel Dekker, Inc.: Ney York, 1996, p 683-717.

(83) Israelachvili, J. N. Intermolecular and Surface Forces; 2nd ed.; Academic Press: San Diego, CA, 1991.

(84) Lipert, R. J.; Lamp, B. D.; Porter, M. D. "Specular Reflection Spectroscopy" In Modern Techniques in Applied Molecular Spectroscopy; Mirabella, F. M., Ed.; Wiley: New York, 1998, p 83-126.

(85) Greenler, R. G. J. Chem. Phys. 1966, 44, 310-5.

(86) Binnig, G.; Quate, C. F.; Gerber, C. Phys. Rev. Lett. 1986, 56, 930-3.

(87) Sarid, D. Scanning Force Microscopy With Applications to Electric, Magnetic and Atomic Forces; Oxford University Press: New York, 1994.

(88) García, R.; Pérez, R. Surf. Sci. Rep. 2002, 47, 197-301.

(89) Takano, H.; Wong, S.-S.; Harnisch, J. A.; Porter, M. D. Langmuir 2000, 16, 5231-3.

(90) Takano, H.; Porter, M. D. J. Am. Chem. Soc. 2001, 123, 8412-3.

(91) Evans, S. D.; Urankar, E.; Ulman, A.; Ferris, N. J. Am. Chem. Soc. 1991, 113, 412131.

(92) Belaidi, S.; Girard, P.; Leveque, G. J. Appl. Phys. 1997, 81, 1023-30.

(93) Hudlet, S.; Saint Jean, M.; Guthmann, C.; Berger, J. Eur. Phys. J. B 1998, 2, 5-10.

(94) Olsson, L.; Lin, N.; Yakimov, V.; Erlandsson, R. J. Appl. Phys. 1998, 84, 4060-4. 


\title{
CHAPTER 2: A SUPRISING ODD-EVEN OSCILLATION IN THE HETEROGENEOUS ELECTRON-TRANSFER RATE OF A SOLUTION-BASED REDOX COUPLE AT ALKANETHIOLATE MONOLAYERS ON GOLD
}

\author{
Grant A. Edwards, Adam J. Bergren, and Marc D. Porter
}

Ames Laboratory-U.S.D.O.E., Department of Chemistry, and the Institute for Combinatorial Chemistry, Iowa State University, Ames, Iowa 50011

A manuscript submitted as a communication to the Journal of the American Chemical Society*

\begin{abstract}
$\underline{\text { Abstract }}$
This paper has examined the rate of heterogeneous electron transfer at alkanethiol-based monolayers $\left(\mathrm{HS}\left(\mathrm{CH}_{2}\right)_{n} \mathrm{CH}_{3}\right)$ of varied chain length by using the solution-based outer-sphere redox couple hydroxymethylferrocene (HMF). Results show, for the first time, that the rate constant $\left(k_{\text {app }}^{0}\right)$ for solution-based couples can exhibit an odd-even dependence on $n$. An extraction of the tunneling constant from these data yield a value much lower than that expected for fully extended alkyl chains. The value is, however, comparable

* This article was submitted as a communication to the J.Am. Chem. Soc. and subsequently rejected. Reviewer concerns were addressed, including additional experimental results, and are contained within Adam Johan Bergren's dissertation entitled Electron transfer reactivity patterns at chemically modified electrodes: fundamentals and application to the optimization of redox recycling amplification systems, Iowa State University, 2006. This finalized manuscript is in preparation for submission to J. Phys Chem.
\end{abstract}


to that found for electron transfer at adlayer defects. This result points to the surprising conclusion that the odd-even dependence reflects contributions from electron transfer at or immediately surrounding defects in the chain structure of the adlayer. Literature evidence supporting this claim is described.

\section{Introduction}

The structure and interfacial properties of alkanethiol-derived $\left(\mathrm{CH}_{3}\left(\mathrm{CH}_{2}\right)_{n} \mathrm{SH}\right)$ monolayers on several metals are closely tied to the polymethylene chain length, $n .^{1-8}$ Wetting ${ }^{6,7}$ and friction, ${ }^{8}$ for example, are influenced by whether $n$ is even or odd. This dependence at gold arises from an oscillation in the methyl group orientation as a consequence of the $\sim 24^{\circ}$ tilt (Figure 1) of the chains with respect to the surface normal. ${ }^{5}$ Thus, the permanent dipole moment of the methyl group, which is closely aligned with the transition dipole of the symmetric methyl stretch, is canted $\sim 53^{\circ}$ when $n$ is even but only $\sim 26^{\circ}$ when $n$ is odd..$^{5,6}$ This effect yields corresponding differences in surface tension, which are manifested in the observed interfacial properties.

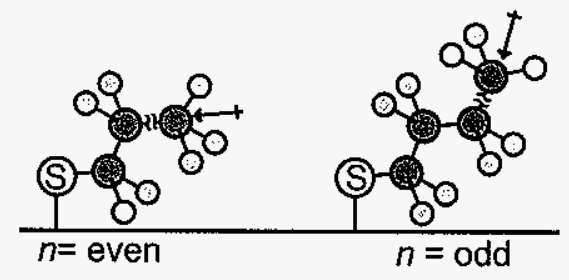

Figure 1. Idealized model illustrating the oscillation of the methyl group orientation for alkanethiolate monolayers on gold.

These findings suggest there may also be an odd-even dependence in the apparent heterogeneous electron-transfer rate constant $\left(k_{\text {app }}^{0}\right)$ for solution-based redox couples at methyl-terminated adlayers. Odd-even oscillations have been observed in $k_{\text {app }}^{0}$ for adlayers with ferrocene groups tethered to the chain terminus. ${ }^{9,10}$ Furthermore, theoretical treatments have predicted an odd-even modulation of long range electron-transfer kinetics across saturated bridges. ${ }^{11-13}$ Such a dependence, however, has not yet been observed for 
solution-based systems. This paper presents the first evidence for such an effect in experiments using the outer-sphere redox couple hydroxymethylferrocene (HMF). We also propose a surprising origin for this observation, drawing in part, on previous theoretical ${ }^{11-13}$. and experimental ${ }^{9,10}$ work.

\section{Experimental}

\section{Chemicals and Reagents}

Distilled water was further purified by deionization with a Millipore Milli-Q water system. Sulfuric acid and sodium fluoride were obtained from Fisher, potassium ferrocyanide from Sigma, and hydroxymethylferrocene (HMF) from Aldrich. Thiols with $n=6-11$ were purchased from Aldrich. Punctilious grade ethanol from Aaper was used to prepare thiol solutions. All chemicals were used as received.

\section{Substrate Preparation}

Substrates for electrochemical measurements consist of polycrystalline gold disk electrodes from Bioanalytical Systems (area $0.02 \mathrm{~cm}^{2}$ ). Procedurally, electrodes were: 1 ) polished with successively finer grades $(1.0,0.3,0.05 \mu \mathrm{m})$ of slurried alumina; 2$)$ sonicated in isopropanol and water for $20 \mathrm{~min}$; and 3) cycled once between 0.0 and $+1.5 \mathrm{~V}\left(50 \mathrm{mV} \mathrm{s}^{-1}\right)$ in $1.0 \mathrm{M} \mathrm{H}_{2} \mathrm{SO}_{4}$ as a final cleaning and surface assessment step. Both the shape of the voltammogram (the presence of only one oxide formation and stripping wave) and the charge under the stripping wave served as metrics for reproducible surface preparation (Figure 2 shows the general shape of the voltammogram using a qualified electrode). ${ }^{14}$ First, a voltammogram exhibiting extraneous features resulted in disqualification of that electrode. Second, the charged measured under the oxide stripping wave was used to assess the area of each electrode, with usable electrodes exhibiting a charge of $28.7 \pm 15.7 \mu \mathrm{C}$. These steps, developed in an ongoing effort to understand the subtleties of surface preparation procedures 
on electrode performance, were key in realizing the ability to detect an odd-even effect on $k_{\text {app. }}^{0}$. Electrodes were rinsed with water and ethanol, and then immersed for $20.0 \mathrm{~min}$ in $1.0 \mathrm{mM}$ ethanolic thiol solutions. After monolayer formation, the electrodes were rinsed well with ethanol, dried in a directed stream of nitrogen, and interrogated with electrochemistry immediately.

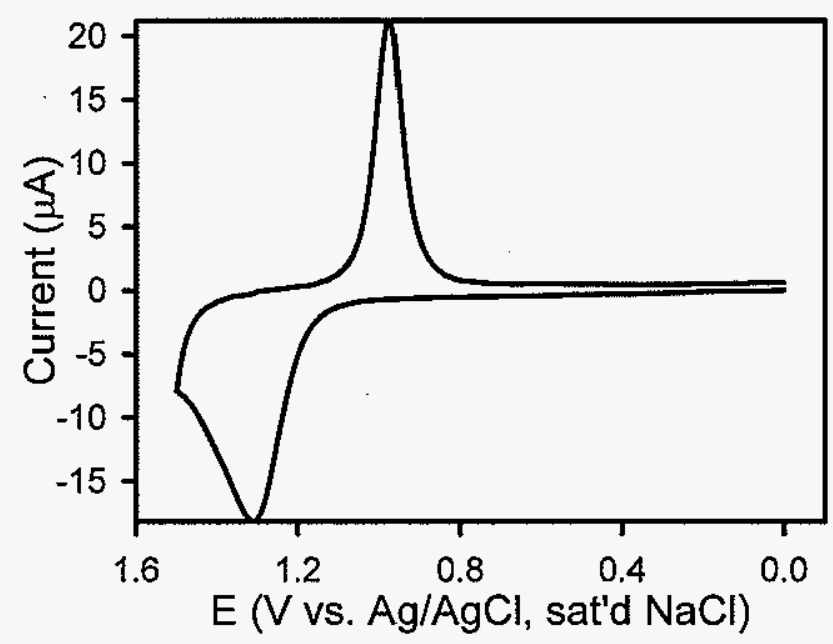

Figure 2. Cyclic voltammogram of $\mathrm{H}_{2} \mathrm{SO}_{4}$ cleaning step. $1 \mathrm{M} \mathrm{H}_{2} \mathrm{SO}_{4}$, Scan Rate $50 \mathrm{mV} \mathrm{s}^{-1}$.

Substrates for infrared reflection spectroscopy (IR-RAS) and wetting measurements were prepared by resistive evaporation of $\sim 15 \mathrm{~nm} \mathrm{Cr}$ (evaporation rate: $0.1 \mathrm{~nm} \mathrm{~s}^{-1}$ ) followed by $300 \mathrm{~nm}$ of $99.9 \%$ purity $\mathrm{Au}$ (evaporation rate: $0.2 \mathrm{~nm} \mathrm{~s}^{-1}$ ) onto clean $1 \times 3$ in. glass microscope slides (Fisher) in an Edwards E306A coating system at a pressure of $7.5 \times 10^{-7}$ torr. Immediately upon removal from the vacuum chamber, the slides were placed into deionized water for storage (all uncoated gold samples were stored for less than $6 \mathrm{~h}$ ). Prior to each IR-RAS measurement, one slide was taken from the storage solution and immersed into a $1.0 \mathrm{mM}$ thiol solution for $20 \mathrm{~min}$, rinsed copiously with ethanol, and dried in a directed stream of nitrogen before being placed into the spectrometer. Directly after each IR-RAS spectrum was acquired ( $\sim 35$ min acquisition time), the same sample was then used for contact angle measurement. 


\section{Electrochemical Measurements}

Electrochemical measurements were carried out using a $\mathrm{CH}$ Instruments model $600 \mathrm{~A}$ potentiostat. Freshly prepared electrodes were used in a three-electrode cell, with a silver/silver chloride (saturated sodium chloride) reference electrode and a flame-cleaned, coiled platinum wire as an auxiliary electrode. All potentials are reported with respect to a silver/silver chloride, saturated sodium chloride electrode $(+0.192 \mathrm{~V}$ vs. NHE). Solutions of $10.0 \mathrm{mM}$ ferrocyanide and 1.00 mM HMF were prepared using a stock solution of aqueous $0.125 \mathrm{M}$ sodium fluoride, which served as the supporting electrolyte. All solutions were purged with nitrogen for $10 \mathrm{~min}$ prior to electrochemical measurements, and a blanket of nitrogen was kept over the solution throughout the experiments. Cyclic voltammograms (CVs) were collected at three different scan rates $\left(50,75\right.$, and $\left.100 \mathrm{mV} \mathrm{s}^{-1}\right)$. The initial potential was set at $-150 \mathrm{mV}$ and the switching potential at $+600 \mathrm{mV}$. Before initiating the first of three cycles, the potential was set at $-150 \mathrm{mV}$ for $10 \mathrm{~s}$ to allow decay of double layer charging current. CVs were collected in three separate solutions: 1) in electrolyte only to serve as background; 2) in the $\mathrm{Fe}(\mathrm{CN})_{6}{ }^{4-}$ solution; and finally 3 ) in the $\mathrm{HMF}$ solution.

Nicholson's method was utilized to translate $\Delta E_{\mathrm{p}}$ to apparent standard heterogeneous electron-transfer rate constants $\left(k_{\text {app }}^{0}\right) .^{15} \Delta E_{\mathrm{p}}$-values were determined accurately within $2 \mathrm{mV}$. A diffusion coefficient of $5.2 \times 10^{-6} \mathrm{~cm}^{2} \mathrm{~s}^{-1}$ and a transfer coefficient $(\alpha)$ of 0.5 were assumed for HMF. Since the voltammograms exhibit shapes diagnostic of diffusion limitations, contributions from adsorbed HMF is negligible.

For an electron-transfer process across a dielectric barrier, the rates fall off exponentially with the thickness of the barrier (Equation 1), where $k_{d=0}^{0}$ is the unimpeded electron transfer rate constant, $k_{\text {app }}^{0}$ is the value of the rate constant for electron transfer across a barrier of thickness $d$, and $\beta$ is the tunneling constant. The value for $\beta$ has been found to be $\sim 1-1.2 \AA^{-1}$ for saturated hydrocarbon barriers. ${ }^{15}$ The thickness of a fully extended alkyl chain tilted $\sim 24^{\circ}$ from the surface normal is expected to increase by $\sim 1.1 \AA$ per 
methylene unit, ${ }^{16}$ and therefore, the corresponding tunneling constant would be $\sim 1 n^{-1}$ if a defect-free thiolate coating was limiting the approach of redox molecules to the electrode.

$$
k_{\mathrm{app}}^{0}=k_{d=0}^{0} e^{-\beta d}
$$

\section{Contact Angle Measurements}

Advancing $\left(\theta_{\mathrm{a}}\right)$ and receding $\left(\theta_{\mathrm{r}}\right)$ contact angles were collected using a Rame-Hart Model 100-00 115 goniometer with deionized water as the probe liquid. A $\sim 5-\mu \mathrm{L}$ drop of water was dispensed onto or removed from the substrate surface using a $10-\mu \mathrm{L}$ Gilson Pipetteman.

\section{Infrared Spectroscopy}

Infrared spectra were acquired with a Nicolet 740 FT-IR spectrometer using $p$-polarized light incident at $80^{\circ}$ equipped with a liquid nitrogen cooled mercury cadmium telluride detector. The sample chamber was purged with nitrogen for 20 min prior to initiating data collection. Spectra were obtained by referencing 1024 sample scans to 1024 background scans at $2 \mathrm{~cm}^{-1}$ resolution (zero filled to $1 \mathrm{~cm}^{-1}$ ) with Happ-Genzel apodization. Background spectra were obtained using a gold slide coated with perdeuterated octadecanethiolate.

\section{$\underline{\text { Results and Discussion }}$}

Infrared reflection spectra (Figure 3) establish that our samples form structurally similar adlayers of different thicknesses with an odd-even oscillation of the methyl group orientation. First, the increase in adlayer thickness is confirmed by the linear increase in the strength for the polymethylene chain modes; this is shown in Figure 4A (squares) for $v_{s}\left(\mathrm{CH}_{2}\right)$ 


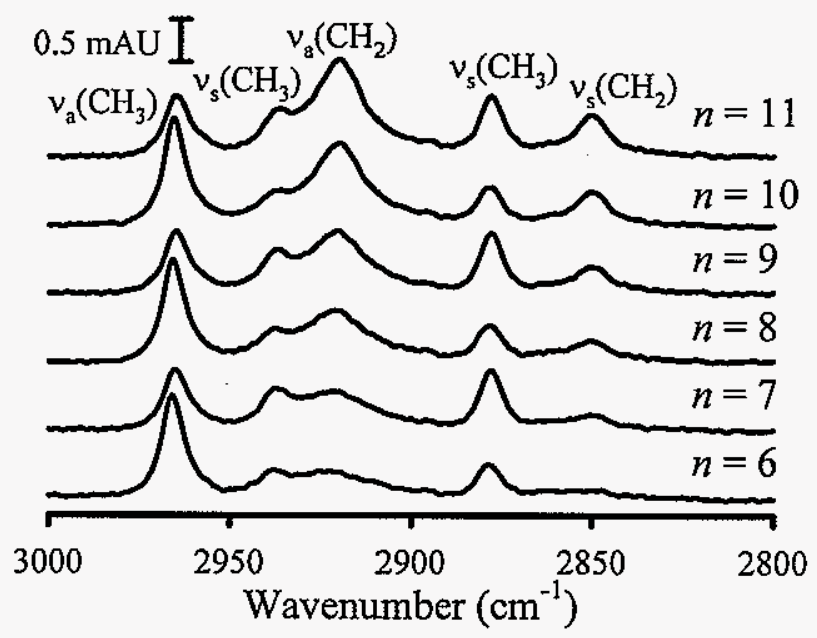

Figure 3. Infrared reflection spectra for alkanethiolate monolayers $(n=6-11)$ on gold. The angle of incidence was $80^{\circ}$ utilizing $p$-polarized light. ${ }^{6}$

$\left(2850 \mathrm{~cm}^{-1}\right)$. Linearity also indicates there is no detectable difference in the average orientation of the polymethylene chains. Second, the strength of the methyl stretches undergoes a saw-tooth variation; this is demonstrated in Figure 4A (circles) for the low energy Fermi resonance mode for $v_{s}\left(\mathrm{CH}_{3}\right)\left(2878 \mathrm{~cm}^{-1}\right)$. Since the absorbance for a vibrational mode is affected by the orientation of its transition dipole with respect to the surface normal on a highly reflective metal, ${ }^{5}$ the small differences in the strength of $v_{\mathrm{s}}\left(\mathrm{CH}_{3}\right)$ for $n=7,9$, and 11 argue that the average orientation of the methyl group for all the odd-numbered adlayers is effectively the same. The same generalization applies to $n=6,8$, and 10 .

Evidence for an odd-even effect on wettability is illustrated by the advancing contact angles $\left(\theta_{\mathrm{a}}\right)$ for water (Figure 4B). While near the precision of the measurement, $\theta_{\mathrm{a}}$ is higher when $n$ is odd. In other words, the odd-numbered layers are more hydrophobic than the even-numbered layers. This trend is attributed largely to a screening of the dispersion forces from the underlying structure by the permanent dipole moment of the methyl group. ${ }^{6,7}$

In testing for an odd-even effect on $k_{\text {app }}^{0}$, several water-soluble redox couples (e.g., substituted ferrocenes and ferrocyanide) and supporting electrolytes were screened. Odd-even correlations were observed with a few systems (e.g., $\mathrm{Fe}\left(\mathrm{CN}_{6}\right)^{4-}$ in $\left.0.125 \mathrm{M} \mathrm{KCl}\right)$, with the 


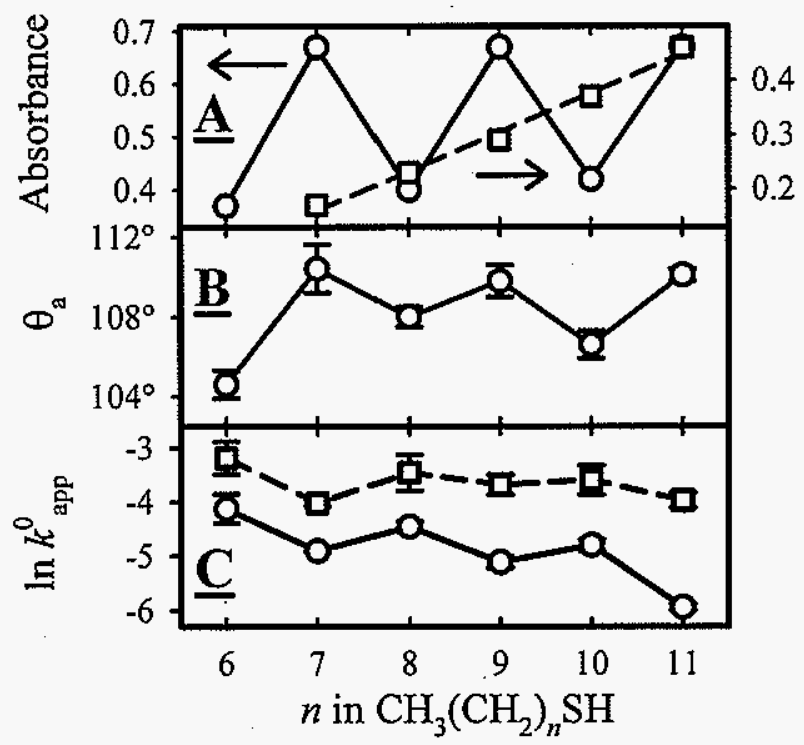

Figure 4. Characterization data for alkanethiolate monolayers on gold: (A) absorbances (mAU) for $v_{\mathrm{s}}\left(\mathrm{CH}_{3}\right)(-)$, and $v_{\mathrm{s}}\left(\mathrm{CH}_{2}\right)(--)$ from infrared reflection spectra (Figure 3); (B) $\theta_{\mathrm{a}}$ of water in air; and (C) $k_{\text {app }}^{0}\left(\mathrm{~cm} \mathrm{~s}^{-1}\right)$ for two separate trials. The length of the error bars are two standard deviation for 3-10 measurements per sample, and are sometimes smaller than the data points.

strongest, most consistent dependence found for $\mathrm{HMF}$ in $0.125 \mathrm{M} \mathrm{NaF}$, which suggests an, as of yet unexplored, effect of redox probe and supporting electrolyte. The cyclic voltammograms from one set of trials are shown in Figure 5. An examination of these data in terms of the voltammetric peak separation $\left(\Delta E_{\mathrm{p}}\right)$, which can be translated to $k_{\text {app }}^{0}$ values, ${ }^{17}$ yields two noteworthy observations.

First and foremost, $\Delta E_{\mathrm{p}}$ exhibits an odd-even dependence on $n$. The corresponding oscillation of $k_{\text {app }}^{0}$ is given in Figure $4 \mathrm{C}$ (solid line). This figure also presents a second set of data (dashed line) to exemplify the reproducibility found for samples prepared a few days apart. While the absolute values of $k_{\text {app }}^{0}$ differ, both data sets show an odd-even dependence on $n$.

Second, the changes in $k_{\text {app }}^{0}$ tend toward preliminary expectations for increases in $n$. That is, the thickness of the barrier to electron transfer should increase as $n$ increases, with a corresponding exponential decrease in $k_{\text {app. }}^{0}{ }^{18,19}$ The decrease in $k_{\text {app, }}^{0}$, however, oscillates 
with respect to $n$. Thus, $k_{\text {app }}^{0}$ for $n=8$ is actually greater than that for $n=7$, but less than that for $n=6$. A parallel statement applies to the trend for $n=9$ through 11, (i.e., $k_{\text {app }}^{0}$ for $n=10$ is greater than $n=9$ and $n=11$ ), noting that $k_{\text {app }}^{0}$ for $n=9$ is less than that for $n=8$.

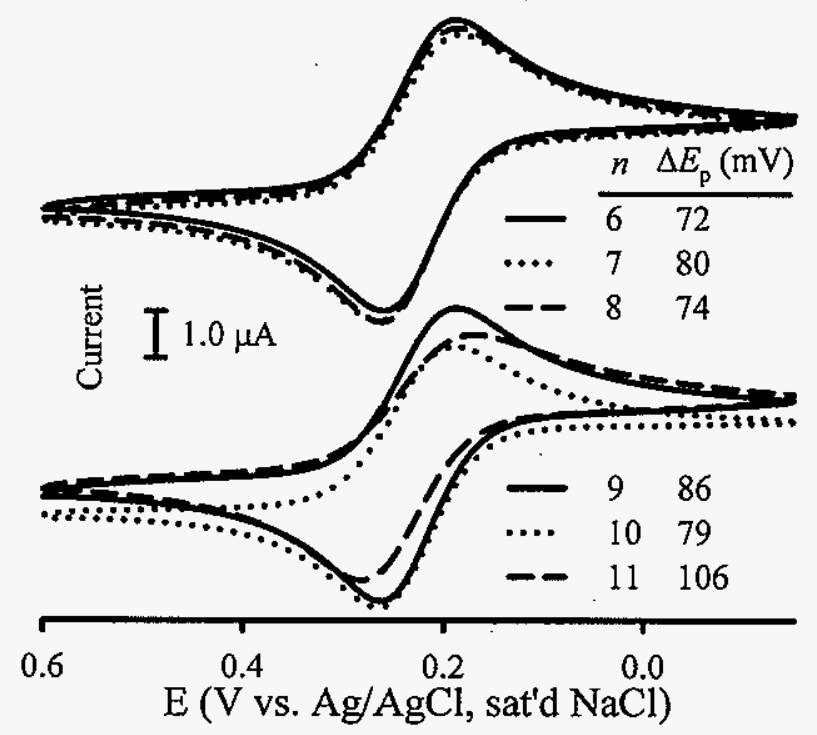

Figure 5. Cyclic voltammograms of $1 \mathrm{mM} \mathrm{HMF}$ in $0.125 \mathrm{M} \mathrm{NaF}$ at alkanethiolate adlayers on gold $(n=6-11)$ : scan rate $=50 \mathrm{mV} \mathrm{s}^{-1}$; geometric electrode area $=0.02 \mathrm{~cm}^{2}$.

What is the origin of our observed odd-even effect? There are several candidates, acting alone or in concert. For instance, the methyl group orientation may affect solvation at the liquid-solid interface. An oscillation in the adlayer thickness $(0.2-0.3 \mathrm{~nm})$ that stems from the difference in the angles of the $\mathrm{CH}_{3}-\mathrm{CH}_{2}$ bond with respect to the surface normal may also play a role. ${ }^{9}$ Another possibility reflects the odd-even dependence of the electronic coupling factor for saturated bridges (i.e., quantum interference). ${ }^{11-13}$

Assessing the importance of these factors is, however, complicated by least squares fits of the two data sets in Figure $4 \mathrm{C}$. This analysis yields tunneling constants $\left(\beta, 0.3 n^{-1}\right.$, solid line and $0.1 n^{-1}$, dashed line) that are smaller than expected for fully extended chains. $\beta$-values for outer-sphere, solution-based couples at fully extended chains are $\sim 1.1 n^{-1}{ }^{20}$ Comparable values are found for ferrocene groups tethered to the chain terminus of such 
systems. ${ }^{9,10}$ Our $\beta$-values are more typical for processes that occur at or in close proximity to adlayer defects (i.e., decreased barrier thickness). ${ }^{3}$ This similarity points to an unexpected possibility: the evolution of $k_{\text {app }}^{0}$ arises from structural variations at or immediately surrounding defects. Evidence from scanning tunneling microscopy studies support this possibility. ${ }^{18,19}$ Some images show the width of the boundary between two ordered domains is consistent with a missing row of adsorbates. ${ }^{19}$ Other images reveal the presence of domains with different chain tilts. ${ }^{18}$ Both examples are intimately connected to the topology of the substrate and the kinetics of adlayer formation. Experiments to probe this interesting phenomenon by further characterization of the structure and electrochemical performance of these and related systems are underway.

\section{Acknowledgments}

This work was supported by Eastman Chemical and the Ames Laboratory-USDOE. G.A.E. gratefully acknowledges a Conoco-Phillips graduate research fellowship. The Ames Laboratory is operated by Iowa State University for the U.S. Department of Energy under Contract W-7405-ENG-82.

\section{$\underline{\text { References }}$}

(1) Bryant, M. A.; Pemberton, J. E. J. Am. Chem. Soc. 1991, 113, 8284-93.

(2) Chidsey, C. E. D.; Loiacono, D. N. Langmuir 1990, 6, 682-91.

(3) Finklea, H. O. "Electroanalytical Methods: Self-Assembled Monolayers on Electrodes" In Encylopedia of Analytical Chemistry; Meyers, R. A., Ed.; John Wiley \& Sons, Ltd: Chichester, 2000; Vol. 11, p 10090-115.

(4) Kim, H. I.; Houston, J. E. J. Am. Chem. Soc. 2000, 122, 12045-6.

(5) Laibinis, P. E.; Whitesides, G. M.; Allara, D. L.; Tao, Y.-T.; Parikh, A. N.; Nuzzo, R. G. J. Am. Chem. Soc. 1991, 113, 7152-67.

(6) Walczak, M. M.; Chung, C.; Stole, S. M.; Widrig, C. A.; Porter, M. D. J. Am. Chem. Soc. 1991, 113, 2370-8.

(7) Wenzl, I.; Yam, C. M.; Barriet, D.; Lee, T. R. Langmuir 2003, 19, 10217-24. 
(8) Wong, S.-S.; Takano, H.; Porter, M. D. Anal. Chem. 1998, 70, 5209-12.

(9) Smalley, J. F.; Feldberg, S. W.; Chidsey, C. E. D.; Linford, M. R.; Newton, M. D.; Liu, Y.-P. J. Phys. Chem. 1995, 99, 13141-9.

(10) Sumner, J. J.; Weber, K. S.; Hockett, L. A.; Creager, S. E. J. Phys. Chem. B. 2000, 104, 7449-54.

(11) Cheng, J.; Miller, C. J. J. Phys. Chem. B 1997, 101, 1058-62.

(12) Hsu, C.-P. J. Electroanal. Chem. 1997, 438, 27-35.

(13) Hsu, C.-P.; Marcus, R. A. J. Chem. Phys. 1997, 106, 584-98.

(14) Dickertmann, D.; Schultze, J. W.; Vetter, K. J. J. Electroanal. Chem. 1974, 55, 42943.

(15) Bard, A. J.; Faulkner, L. R. Electrochemical Methods: Fundamentals and Applications; 2nd ed.; John Wiley \& Sons, Inc.: New York, NY, 2001.

(16) Porter, M. D.; Bright, T. B.; Allara, D. L.; Chidsey, C. E. D. J. Am. Chem. Soc. 1987, $109,3559-68$.

(17) Nicholson, R. S. Anal. Chem. 1965, 37, 1351-5.

(18) McDermott, C. A.; McDermott, M. T.; Green, J.-B.; Porter, M. D. J. Phys. Chem. $1995,99,13257-67$.

(19) Poirier, G. E.; Pylant, E. D. Science 1996, 272, 1145-8.

(20) Miller, C.; Cuendet, P.; Gratzel, M. J. Phys. Chem. 1991, 95, 877-86. 


\title{
CHAPTER 3: MOLECULAR MODELING OF n-ALKANETHIOLATE MONOLAYERS ON GOLD: ADLAYER ORIENTATION EFFECTS
}

\author{
Grant A. Edwards, Adam J. Bergren, Erik J. Cox, and Marc D. Porter \\ Ames Laboratory-US D.O.E., Department of Chemistry, and the Institute for Combinatorial \\ Discovery, Iowa State University, Ames, Iowa 50011
}

\begin{abstract}
$\underline{\text { Abstract }}$
The fabrication of $n$-alkanethiolate $\left(\mathrm{CH}_{3}\left(\mathrm{CH}_{2}\right)_{n} \mathrm{SH}\right)$ monolayers on gold is a facile method for creating modified interfaces in areas spanning molecular electronics, sensors, biomaterials, and tribology. This paper details an examination of experimental results (ellipsometric thicknesses and contact angles) with structural comparisons to predictions from molecular modeling. The effects of adlayer chain tilt $(\alpha)$ and twist $(\beta)$ on the expected thickness and dipole moment of the film are emphasized. The dipole moment is used to correlate the corresponding wettability changes in terms of adlayer orientation and thickness. The change in the dipole moment of the adlayer with increasing chain length correlates with the evolution of the wettability data, suggesting the adlayer length and orientation dictate the observed contact angle. The modeled thickness is compared directly to ellipsometric thickness measurements. The experimental thickness increase with each additional methylene unit is $1.14 \AA$, which corresponds to an approximate chain tilt of $30^{\circ}$. This chain tilt is corroborated by values determined using infrared reflection absorption spectroscopy. In addition, the experimental and model data present surprising odd-even variations in contact angle and thickness. The overall agreement between the experimental measurements and
\end{abstract}


model results for both contact angle and thickness infer that molecular modeling is a vital methodology for exploration of the structural details of interfaces.

\section{Introduction}

Use of self-assembled $n$-alkanethiolate monolayers on gold $\left(\mathrm{CH}_{3}\left(\mathrm{CH}_{2}\right)_{n} \mathrm{~S}-\mathrm{Au}\right)$ is widespread. The early works in this area ${ }^{1-4}$ and subsequent use in constructing well-defined organic surfaces and interfaces ${ }^{5-7}$ has lead to a large number of publications. Many of these studies investigated the structural characteristics of this adlayer system using techniques including: diffraction, ${ }^{8-16}$ ellipsometry, ${ }^{1,3,17-21}$ temperature programmed desorption, ${ }^{4,18,19}$ wettability, ${ }^{1,18-25}$ X-ray photoelectron spectroscopy, ${ }^{4,17-21,23,26}$ infrared reflection absorption spectroscopy, ${ }^{1,3,4,17,19,20,27,28}$ surface Raman scattering, ${ }^{29,30}$ scanning tunneling and atomic force microscopy, ${ }^{3,15,31-39}$ electron spectroscopies, ${ }^{4}$ and electron microscopy. ${ }^{14,17,18,20,40} \mathrm{~A}$ result of these characterizations is a broadly accepted structural model for these adlayers. The sulfur atom binds to the gold resulting in a gold thiolate. ${ }^{23,41,42}$ At $\mathrm{Au}(111)$, the thiolates are presumably bound in the threefold hollow sites, ${ }^{43,44}$ although this has not been experimentally verified. The monolayer also readily forms a $(\sqrt{3} \times \sqrt{3}) \mathrm{R} 30^{\circ}$ overlayer,,$^{8-10,25,31,35,40,45}$ in which the polymethylene chains contain a large percentage of all-trans segments. ${ }^{20}$ The tilt $(\alpha)$ and twist $(\beta)$ of the chains are presented schematically in Figure 1, where $\alpha$ is the tilt of the C-C chain backbone with reference to the surface normal, and $\beta$ is the twist of the $\mathrm{C}-\mathrm{C}$ chain with respect to a plane established by the chain axis and the surface normal. ${ }^{6} \mathrm{At} A \mathrm{Au}(111)$, the most commonly reported experimental values of $\alpha$ and $\beta$ are $\sim 30^{\circ}$ and $\sim 55^{\circ}$, respectively. ${ }^{3,6,19,20,27,46}$

Although little is known about the Au-S bonding interactions in thiolate systems, for the purpose of this manuscript, we assume that the $\mathrm{Au}-\mathrm{S}$ bond or interaction can be discussed as an interaction of the sulfur to a surface plane of gold atoms. ${ }^{20,29,47}$ In this way, the Au-S bond is defined as oriented in the surface normal. 


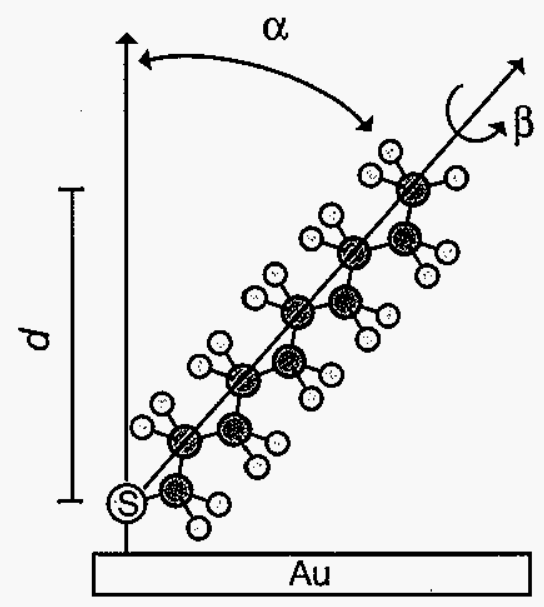

Figure 1. Schematic side view of an alkanethiolate $(n=9)$. The tilt $(\alpha)$ and twist $(\beta)$ angles are described in the text.

In addition to discussing the idealized structure of these adlayer systems, research has also detailed the effect of chain length. ${ }^{3,17,18,20-23,29,48-55}$ For example, ellipsometry has been utilized to interrogate thicknesses as a function of chain length $(n){ }^{3,17,18,21}$ Porter et al. ${ }^{3}$ interrogated an odd-chain series. These data exhibited nonlinearity when $n<10$. However, at $n>10$, the thickness increases linearly, with a slope of $1.5 \AA n^{-1}$. Interestingly, this slope is larger than expected for an all-trans chain oriented normal to the surface; when $\alpha$ is $25^{\circ}$, the expected slope is $1.1 \AA n^{-1}$. Bain ${ }^{18}$ et al. also reported thickness values for a large number of chain lengths and found a slope of $1.5 \AA n^{-1}$. In both cases, possible explanations for the discrepancy between the expected and experimental slope were presented, including refractive index variations with $n$, contaminates on the gold substrate or adlayer, and the tensor nature of the refractive index.

Contact angle measurements have also been utilized to probe chain length effects on interfacial tension. ${ }^{18,20-23,48}$ Bain $^{18}$ et al. used a variety of probe liquids to interrogate wetting properties as a function of $n$. These studies revealed that short chain lengths are hydrophilic, with longer chains yielding more hydrophobic surfaces. Laibinis ${ }^{20}$ et al. compared the contact angle of a homologous series of thiolates prepared at gold, copper, and silver surfaces, and Walczak et al. ${ }^{53}$ completed an extensive study of thiolates on silver surfaces. These data 
exhibited the same decrease in contact angle for short chain lengths $(n \leq 8)$. This decrease was rationalized by assuming a more disordered structure for the shorter chain adsorbates that allowed interactions of the probe liquid with the underlying substrate. Bain and Whitesides ${ }^{22}$ investigated the depth sensitivity of contact angle in an attempt to quantitate these effects, and found that between water, glycerol, and hexadecane, water has the greatest depth sensitivity for a buried ether group. This study lends credence to the idea that water as a probe liquid may be sensing the underlying gold at short chain lengths.

In earlier work, Shafrin and Zisman ${ }^{48}$ investigated the effect of perfluorinating methylene units on fatty acids in a study that suggested: 1) the dipole moment of bonds close to the surface control the resulting contact angle; and 2) strong intramolecular dipoles buried within a layer at sufficient distance from the interface may not affect the contact angle. For example, the wettability of chromium surfaces modified by $\mathrm{F}\left(\mathrm{CF}_{2}\right)_{\mathrm{X}}\left(\mathrm{CH}_{2}\right)_{16} \mathrm{COOH}$ and $\mathrm{F}\left(\mathrm{CF}_{2}\right)_{\mathrm{X}} \mathrm{COOH}$ were compared. Both adlayers have similar surface structures, with the carboxylic acid coupling to chromium, and the perfluorinated chain extending away from the surface. When $\mathrm{X}$ was less than 7 , the contact angles were different for the two surfaces; however, when $X=7$ or larger, the values became indistinguishable. This set of experiments also argues that the probe liquid has a finite depth sensitivity.

A number of examples of surprising odd-even chain length dependencies have also been observed, including: friction, ${ }^{50,55}$ argon ion scattering, ${ }^{49}$ heterogeneous electron-transfer kinetics, ${ }^{51,52}$ infrared reflection absorption spectroscopy, ${ }^{20,53,54}$ Raman, ${ }^{29,30} \mathrm{UV}$ photoemission, ${ }^{56}$ and wettability. ${ }^{53,54}$ As an example, for related systems, the oscillation in contact angle ${ }^{53,54}$ was attributed to the influence of surface dipole orientation. In addition, we have observed the odd-even oscillation in heterogeneous electron-transfer kinetics for the solution-based redox molecule hydroxymethylferrocene (see Chapter 2). While there are a number of explanations for the odd-even oscillations (e.g., a thickness oscillation with $n$ ); an all encompassing description has yet to be fully developed and tested. 
Other laboratories have proposed an investigation of the role of the dipole moment in affecting the interfacial properties of these systems is warranted. For example, Alloway et al..$^{56}$ used the calculated dipole moment of the adlayers in an UV-photoelectron spectroscopy study. In addition, Evans and Ulman ${ }^{57}$ measured an apparent dipole of the $n$-alkanethiolate system using the Kelvin surface potential technique, which over a range of $n=4-22$ presented a linear trend. As part of our interest in this area, this work describes the results of an extensive molecular modeling study of the dipole moment as a function of chain length and orientation (i.e., tilts and twists). Since the dipole magnitude is known to affect the surface normal electric field, and consequently the dipole-dipole interactions between the surface and contacting liquid, ${ }^{58}$ a wettability change will result. Therefore, the modeled dipole moment is compared to the observed trend in contact angle values. A surprising result is observed: the model and experimental data exhibit a clear and correlated odd-even oscillation with respect to chain length.

In this light, we also propose a reexamination of the thickness evolution with chain length. A theoretical thickness oscillation was proposed for ferrocene-terminated monolayers as part of an explanation for the dependence of the electron-transfer rate of the tethered ferrocene group. ${ }^{51,59}$ In this case, the thickness was modeled at one chain orientation, as the distance from the electrode surface to the Fe center. In contrast, the work herein uses molecular modeling to predict the thickness for a range of additional conformations of $n$-alkanethiolates on gold. Importantly, the calculated values exhibit a clear, but small odd-even oscillation in thickness with $n$ at various orientations. Interestingly, a reassessment of optical ellipsometric measurements subtly hint at an identical oscillation, and the slope of thickness vs. $n$ matches the expected overall increase in thickness predicted by the modeling data for an expected chain conformation of $\alpha=\sim 30^{\circ}$ and $\beta=\sim 55^{\circ}$ as inferred in other experiments. ${ }^{3,6,19,20,27,46}$ 


\section{Experimental}

\section{Chemicals and Reagents}

Distilled water was further purified by deionization with a Millipore Milli-Q water system. All thiols were provided by Aldrich. Thiol purity was assessed by gas chromatography/mass spectrometry (GC/MS), ${ }^{60}$ and found to exceed $95 \%$ in all cases. The identified impurities are not expected to affect the quality of the resulting adlayers. Punctilious grade ethanol (Aaper) was used to prepare thiol solutions.

\section{Substrate Preparation}

Gold substrates were prepared by resistive evaporation of $\sim 15 \mathrm{~nm}$ chromium followed by $\sim 300 \mathrm{~nm}$ gold on clean glass microscope slides (Fisher Scientific) in a turbomolecular pumped E306A Edwards Coating System (pressure of $<7.5 \times 10^{-5}$ Torr). The evaporation rates were 0.1 and $0.2 \mathrm{~nm} \mathrm{~s}^{-1}$ for chromium and gold, respectively. Assessments by the anodic stripping of adsorbed iodine $\mathrm{e}^{61,62}$ yielded a roughness factor of $1.1 \pm 0.1$. Underpotential deposition and stripping of lead ${ }^{63,64}$ was utilized to determine crystallinity of the gold. This analysis showed that $46 \%$ of the surface is composed of the (111) crystal face, and that terraces of less than 11 atoms across comprise $33 \%$ of the (111) surface texture. The bulk of the remaining $54 \%$ of the surface is composed of other crystal faces or step edges.

\section{Monolayer Deposition}

The $n$-alkanethiolate assemblies were formed by immersion of freshly prepared gold substrates into $1.0 \mathrm{mM}$ ethanolic thiol solutions for $\sim 12 \mathrm{~h}$. Upon removal from solution, the films were rinsed with ethanol and dried with high purity nitrogen.

\section{Wettability}

Contact angles $(\theta)$ were measured using a Rame-Hart model 100 goniometer. A $\sim 5-\mu \mathrm{L}$ drop of deionized water was dispensed onto the substrate surface using a 10- $\mu \mathrm{L}$ Gilson 
Pipetman. Advancing contact angles $\left(\theta_{a}\right)$ were determined after the pipette tip was slowly pulled away from the drop while increasing its volume, while the receding contact angles $\left(\theta_{\mathrm{r}}\right)$ were assessed after slowly pulling the pipette tip away from the drop while decreasing its volume. $^{53}$

\section{Ellipsometry}

Ellipsometric thickness measurements were carried out using an Optrel Multiskop GBR ellipsometer in null mode, with a single wavelength $(632.8 \mathrm{~nm})$ light source at a $70^{\circ}$ angle of incidence. For each substrate, five spots on each of three different samples (15 measurements) were used to determine the average and standard deviations of $\Delta$ and $\Psi$. For uncoated $\mathrm{Au}$, these measurements yielded $\Delta=109.5( \pm 0.1)$ and $\Psi=43.80( \pm 0.05)$, giving a real and imaginary refractive index of 0.168 and -3.527 , respectively, via a classical two-phase, parallel-layer model. For monolayer-coated samples, a three-phase, parallel-layer model was used with the previously measured refractive index of gold and the $\Delta$ and $\Psi$ values (with associated error) for each adlayer. The refractive index for the adlayers was set at $1.50 .^{65}$

\section{Molecular Modeling}

Molecular models of the $n$-alkanethiols $(n=3-17)$ were created by energy minimization using Chem3D Ultra $8.0^{66}$ with the CS MOPAC Pro package. This software employs the Parameterized Model (revision 3, PM3) to generate a potential energy function with a closed shell wave function. The investigation of adlayer orientation required variation of $\alpha$ and $\beta$ over the expected range of $0^{\circ}$ to $90^{\circ}$. Therefore, the bond angle of the H-S-C bond and the dihedral angle of the H-S-C-C bonds were altered to achieve the desired $\alpha$ and $\beta$, using the S-H linkage as reference of the surface normal. In this way, the thiol group was modeled such that the H-S bond is perpendicular to the gold surface. We add that the presence of the sulfhydryl hydrogen $(\mathrm{S}-\mathrm{H})$ is not intended to suggest its presence after adlayer 
formation. In a thiolate adlayer, the S-H bond would be replaced by the S-Au interaction. The alkane chain is then oriented, in all our models, as an all-trans collection of the requisite number $(n)$ of methylene units culminating in the same enforced alignment for the terminal methyl group.

The evolution in adlayer thickness $(d)$ as a function of $n$ and chain orientation ( $\alpha$ and $\beta$ ) was modeled as depicted in Figure 1. Thickness was calculated as the distance between the sulfur atom and the terminal methyl carbon only in the direction of the S-H bond. Although, this calculation does not directly correspond to the actual thickness of the monolayer due to the neglect of the S-Au and methyl C-H bonds, this model would effectively serve as a methodology to predict trends in ellipsometric measurements. The associated differences between the actual adlayer thickness and the modeled values presented here are discussed in the next three paragraphs.

The discrepancy established by treating the Au-S interaction as that of a S-H bond introduces a systematic decrease in modeled thickness. Investigations of thiolate adsorption on Au nanoclusters include calculations that propose a Au-S bond length of about $2.4 \AA{ }^{67}$ Since the tabulated thickness values included here do not include the S-H bond length, the actual modeled thickness would increase approximately $2.4 \AA$ in all cases.

The effect of neglecting the methyl C-H bond is, again, to increase the expected thickness from that proposed by the model. The rotation of the methyl group about the $\mathrm{CH}_{2}-\mathrm{CH}_{3}$ bond is known to occur for $n$-alkanes in the solid state at $150 \mathrm{~K},{ }^{68}$ and therefore, we surmise that the terminal methyl group of the monolayer is also able to rotate. In addition to this rotation, the orientation of the methylene chain also influences the difference in the actual vs. model thickness. When the terminal methyl group is oriented in such a way that the $\mathrm{C}-\mathrm{H}$ bond is parallel to the surface normal, the effective disparity would be of approximately $1.12 \AA$ (the total length of the $\mathrm{C}-\mathrm{H}$ bond). As the $\mathrm{CH}_{2}-\mathrm{CH}_{3}$ bond tilts either direction from this position, the thickness discrepancy would decrease. 
Therefore, the estimated systematic differences of modeling the thiolate monolayer in the way presented by this manuscript (neglecting the Au-S and methyl C-H bonds) would lead to a systematic decrease in magnitude of at most $\sim 3.4 \AA$. We have chosen to disregard these disparities, as the effect on the observed trends in thickness will be minimally affected. In addition, the systematic errors in ellipsometric thickness measurements are of comparable magnitude.

Dipole moments were calculated for each orientation with the CS MOPAC Pro package using the Mulliken charge approximation. The three-dimensional molecular coordinates, in conjunction with the dipole vectors, were utilized to determine the magnitude of the dipole moment (parallel to the alignment of the S-H bond and, therefore, parallel to the surface normal of the adlayers. Although the MOPAC software modeled the thiol precursors and not gold-bound thiolates, the calculated dipole moment should be directly proportional to that of the corresponding adlayer. First, if one takes into account the bond length of $\mathrm{Au}-\mathrm{S}$ $(2.4 \AA)^{67}$ and the charge of 0.2 on the sulfur, ${ }^{57,60}$ an expected dipole of 2.3 Debye results. A simplified analysis of the $\mathrm{H}_{2} \mathrm{~S}$ bond angle $\left(92.1^{\circ}\right)^{69}$ and molecular dipole moment (0.97 Debye $)^{69}$ results in a H-S dipole of 1.4 Debye. Although not a strict representation of the actual H-S dipole of a thiol molecule, it will serve as an illustrative example. In this way, the dipole of the thiolate molecule is approximately one Debye larger in magnitude that the results presented here. In addition, the $\mathrm{Au}-\mathrm{S}$ bond characteristics are consistent with $n$ as evidenced by X-ray photoelectron spectroscopy, ${ }^{60}$ suggesting that the contribution of this dipole will be independent of chain length. 


\section{$\underline{\text { Results and Discussion }}$}

\section{Molecular Modeling}

\section{Thickness}

The impacts of the chain tilt and twist on thickness are summarized in Figure 2, using the structural description in Figure 1. Figure 2A shows the thickness as function of $\alpha$ with $\beta$ fixed at $50^{\circ}$, while Figure $2 \mathrm{~B}$ presents an analogous series for which $\alpha$ is held constant at $30^{\circ}$ with varying $\beta$. These values of $\alpha$ and $\beta$ were chosen not only for illustrative purposes, but also from the structural descriptions in earlier reports, $\alpha=30^{\circ}$ and $\beta=50^{\circ} .3,6,19,20,27,46$ The trends discussed in relation to these data sets are also observed in calculations for other variations in the adlayer structure, such as $\alpha=40^{\circ}$ and $\beta=40^{\circ}$, but are omitted in the interest of space. Linear regression analyses of the data sets are presented in Tables 1 and 2 . The presented slopes are a measure of the overall thickness increase with chain length. In addition, the evolution of the slope with changes in $\alpha$ and $\beta$ illustrate the dependence of the overall thickness on adlayer orientation. The two intercepts (odd and even) for each conformation are a measure of the magnitude of the saw-tooth oscillation that will be discussed in further detail below.

Examination of Figure 2 yields several important observations. First, the adlayer thickness decreases, as expected, with decreasing $n$. Second, the thickness also exhibits a saw-tooth oscillation with $n$ for most orientations (i.e., values of $\alpha$ and $\beta$ ). This oscillation is a manifestation of the orientation of the $\mathrm{CH}_{2}-\mathrm{CH}_{3}$ bond. The bond is oriented more along the surface normal when $n$ is odd-valued as opposed to when $n$ is even-valued. Therefore, the increase in thickness when incrementing $n$ of an even-valued chain by one is larger than when adding a methylene unit to an odd-length chain. As an example, when adding a methylene 

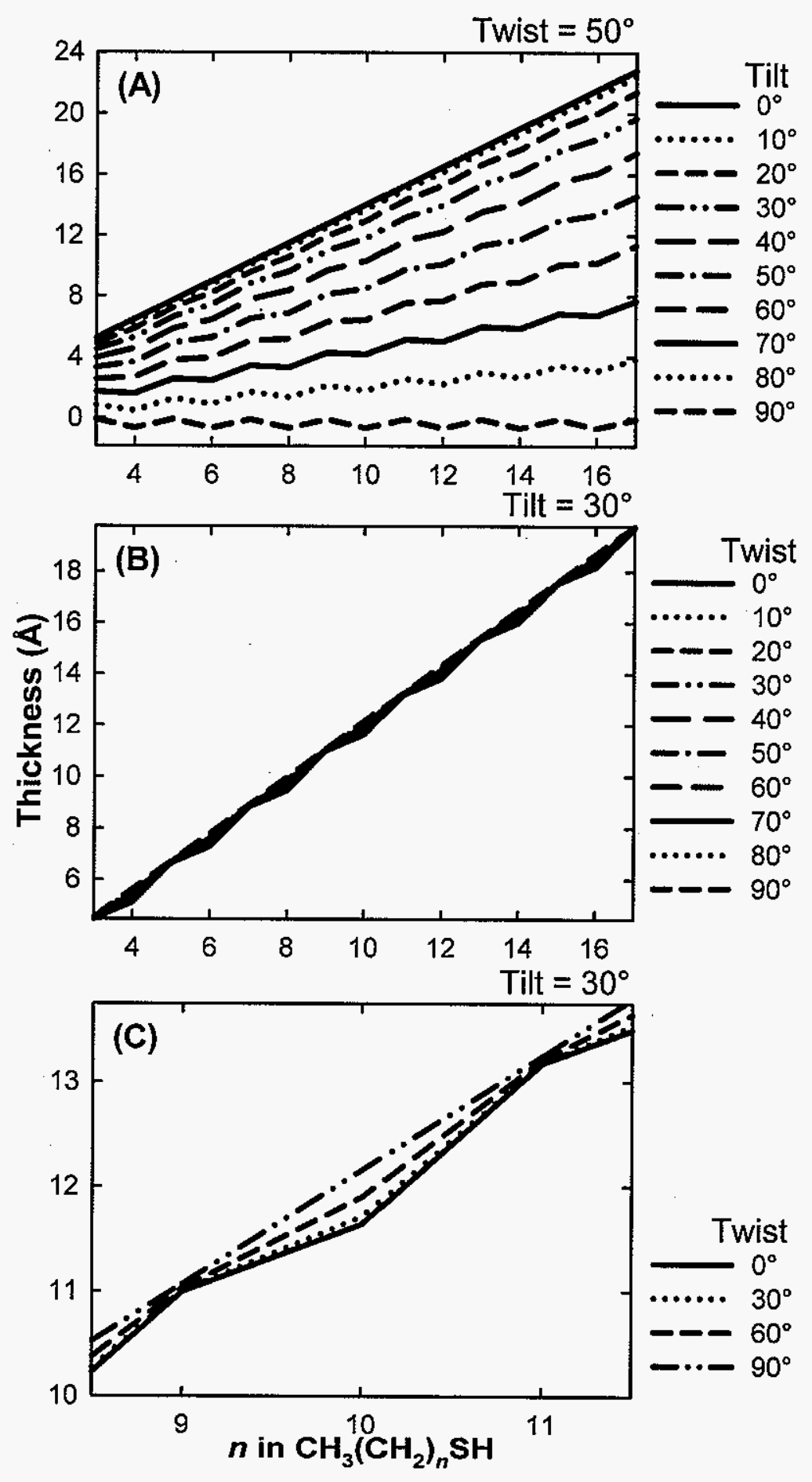

Figure 2. Modeled adlayer thickness as a variation of tilt and twist of the $n$-alkane chain: (A) $\beta$ of $50^{\circ}$ with varying amount of $\alpha,(\mathrm{B})$ and (C) $\alpha$ of $30^{\circ}$ with varying levels of $\beta$. 
Table 1. Linear Regression analysis of the modeled adlayer thickness as a variation of $\alpha$, with $\beta$ of $50^{\circ}$.

\begin{tabular}{llrrrrrrrrrr}
\hline \multicolumn{2}{c}{$\alpha$} & $0^{\circ}$ & $10^{\circ}$ & $20^{\circ}$ & $30^{\circ}$ & $40^{\circ}$ & $50^{\circ}$ & $60^{\circ}$ & $70^{\circ}$ & $80^{\circ}$ & $90^{\circ}$ \\
\hline $\begin{array}{l}\text { Slope } \\
\text { Intercept }\end{array} \quad(\AA)$ & $\left(\AA n^{-1}\right)$ & 1.26 & 1.24 & 1.18 & 1.09 & 0.96 & 0.81 & 0.63 & 0.43 & 0.22 & 0.00 \\
$\quad$ & & & & & & & & & & \\
$\quad$ Odd & 1.46 & 1.42 & 1.34 & 1.22 & 1.06 & 0.87 & 0.65 & 0.41 & 0.16 & -0.09 \\
$\quad$ Even & 1.46 & 1.32 & 1.15 & 0.93 & 0.69 & 0.43 & 0.16 & -0.12 & -0.40 & -0.66 \\
\hline
\end{tabular}

Table 2. Linear Regression analysis of the modeled adlayer thickness as a variation of $\beta$, with $\alpha$ of $30^{\circ}$.

\begin{tabular}{cccccccccccc}
\hline \multicolumn{2}{c}{$\beta$} & $0^{\circ}$ & $10^{\circ}$ & $20^{\circ}$ & $30^{\circ}$ & $40^{\circ}$ & $50^{\circ}$ & $60^{\circ}$ & $70^{\circ}$ & $80^{\circ}$ & $90^{\circ}$ \\
\hline $\begin{array}{l}\text { Slope } \\
\text { Intercept }\left(\AA n^{-1}\right)\end{array}$ & 1.09 & 1.09 & 1.09 & 1.09 & 1.09 & 1.09 & 1.09 & 1.09 & 1.09 & 1.09 \\
$\quad$ & & & & & & & & & & \\
$\quad$ Odd & 1.20 & 1.20 & 1.20 & 1.20 & 1.21 & 1.22 & 1.23 & 1.24 & 1.25 & 1.27 \\
$\quad$ Even & 0.75 & 0.76 & 0.78 & 0.82 & 0.87 & 0.93 & 1.01 & 1.09 & 1.18 & 1.27 \\
\hline
\end{tabular}

unit to an odd-length chain, with a tilt of $30^{\circ}$ and a twist of $50^{\circ}$, the thickness increases $\sim 0.8 \AA$ as opposed to $\sim 1.4 \AA$ when adding a methylene unit to an even chain.

The odd-even oscillation becomes more pronounced as $\alpha$ increases. This trend corresponds directly to the effect of $\alpha$ on the directionality of the $\mathrm{CH}_{2}-\mathrm{CH}_{3}$ bond. As $\alpha$ decreases, the chain becomes more aligned towards the surface normal, which dampens the odd-even difference in bond directionality. Chain twist also dictates the amount of saw-toothed character, as shown in Figure 2B. As $\beta$ approaches $90^{\circ}$, the discrepancy in orientation of the terminal $\mathrm{CH}_{2}-\mathrm{CH}_{3}$ bond orientation with respect to the surface normal becomes smaller and eventually indistinguishable.

\section{Dipole Moment}

The dipole moment of the monolayer has important contributions to many interfacial properties including wettability, adhesion, and friction. Molecular modeling is used to calculate the magnitude of the dipole moment for a series of thiols. These data are discussed in terms of dipole magnitude in the direction of the surface normal $\left(\mu_{\perp}\right)$. The observed trends in $\mu_{\perp}$ will be correlated with contact angle measurements since the increased dipole magnitude results in a higher electric field normal to the surface, which increases 
dipole-dipole interactions with the contacting liquid, and therefore results in a decrease in the contact angle. ${ }^{58}$

The $\mu_{\perp}$ is a combination of the individual dipoles of the thiol molecule, which are comprised of three main components, those of the sulfhydryl hydrogen/sulfur, the terminal methyl group, and the methylene chain. An illustrative schematic of these dipole moments is presented in Figure 3 for an odd- and even-chain length adlayer on a gold surface.

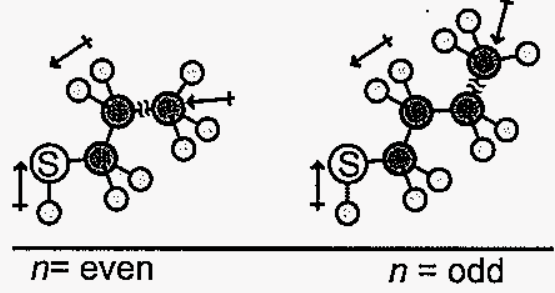

Figure 3. Dipole orientation of odd and even values of chain length.

As a starting point, the S-H dipole is included in the calculation of $\mu_{\perp}$. In an actual thiolate adlayer system, the hydrogen would be replaced by the Au surface, which results in an estimated charge of $\sim-0.2$ on the sulfur. ${ }^{57,60}$ Therefore, the bond dipole from the gold-sulfur interaction will lie in the same direction as that for the S-H linkage, but will be larger in magnitude. In addition, the contribution from this discrepancy is chain length independent. This assertion is supported by the positions of the $S(2 p)$ couplet in X-ray photoelectron spectroscopy, which are virtually identical for thiol-based adlayers of differing chain lengths. ${ }^{60}$ This claim also applies to contributions from image dipoles created in the substrate by the S-Au dipole. These discrepancies will introduce systematic errors, as discussed in the Experimental section, in the magnitude of $\mu_{1}$; however, the observed trends within the data should not be affected.

The orientation of the dipole moment exhibited by the methylene chain is dictated, however, by the tilt and twist of the adlayer. The orientation of the dipole of the methyl group is also dependant upon the orientation, as well as the odd-even character of the methylene 
chain. This orientation dependence follows the same set of variables detailed in the thickness section.

The $\mu_{\perp}$ of the molecule is a vector summation of the individual dipole moments, which can be calculated at various adlayer tilt and twist values. The magnitude of this vector in the direction of the surface normal is plotted in Figure 4 as a function of chain length and orientation. Figure $4 \mathrm{~A}$ shows a $\beta=50^{\circ}$ with varying $\alpha$, and Figure $4 \mathrm{~B}$ presents an analogous series for which $\alpha$ is $30^{\circ}$ and $\beta$ is systematically altered. Negative values represent the directionality of the dipole vector, with the negative end of the dipole located on the methyl group, and the positive end toward the gold surface.

Overall, as $\alpha$ increases, $\mu_{\perp}$ assumes values that are more negative. This result indicates that as the chain moves closer to the surface parallel, the dipole of the methylene chain and methyl group do not contribute as much to $\mu_{\perp}$. In effect, they counterbalance the dipole due to the $\mathrm{S}-\mathrm{H}$ bond to a smaller degree. As the structure approaches a tilt of $0^{\circ}$, the methylene chain and methyl group dipole moments overwhelms the S-H dipole, pushing $\mu_{\perp}$ to positive values (in effect reversing the direction of the dipole moment).

Most of the data in Figure 4 display an increase in the dipole strength when $n<\sim 8$. This trend implies that the methylene chain is responsible for counteracting a portion of the $\mathrm{S}-\mathrm{H}$ dipole. As the length of the methylene chain increases, $\mu_{\perp}$ reaches a limiting value as the dipole contributions due to the chain become effectively constant. This phenomenon is the effect controlling the decrease in contact angle at short chain lengths (see below).

The saw-toothed oscillation in $\mu_{\perp}$ as $n$ increases is most dramatic for chain twists near $0^{\circ}$. In all cases, even-valued chains present a larger $\mu_{\perp}$. Note, this oscillation in dipole suggests an odd-even variation in contact angle value should be observed. However, this trend is obscured at shorter chain lengths $(n \leq 8)$ due to the concurrent overall increase in magnitude as discussed above. 


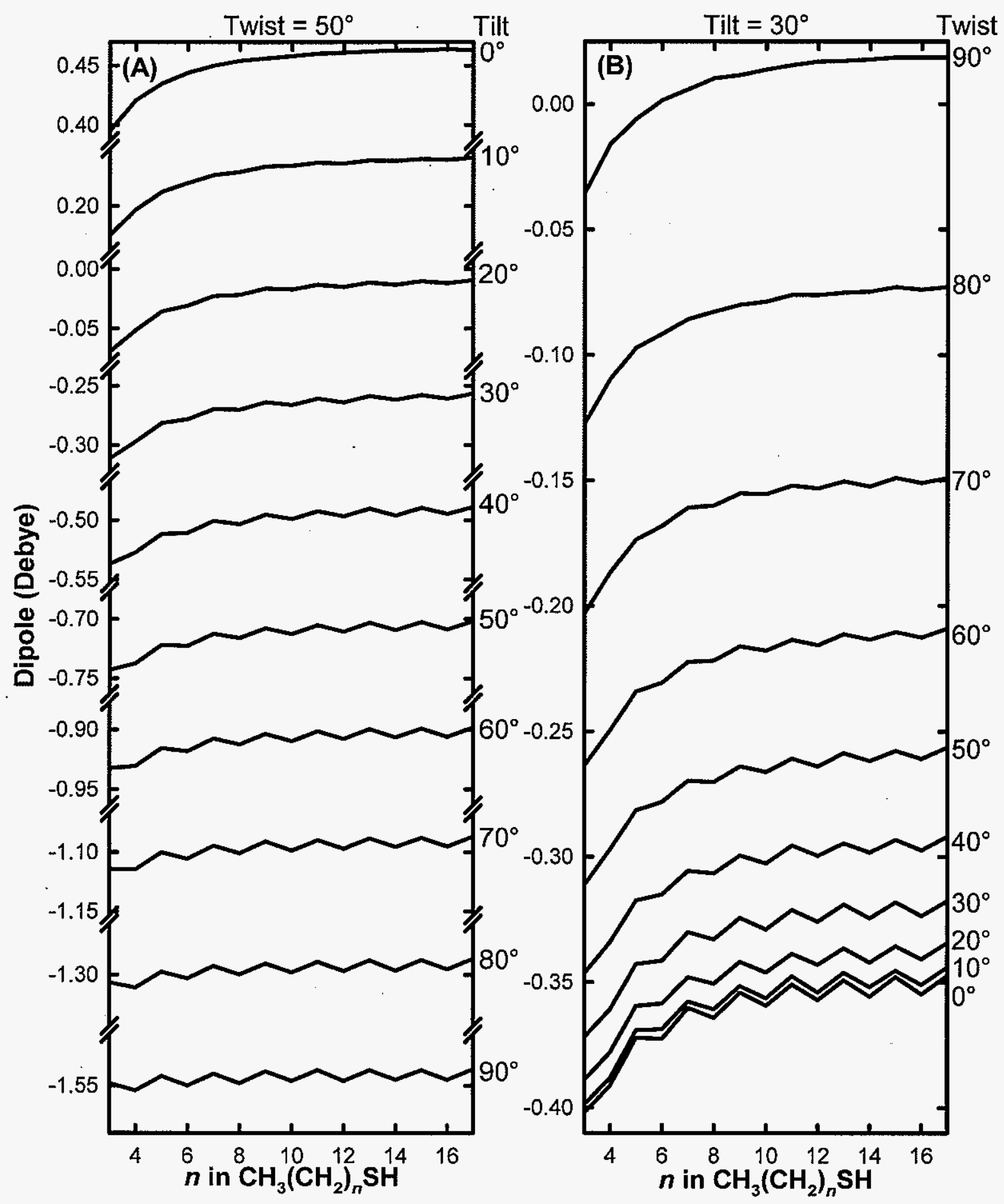

Figure 4. Modeled $\mu_{\perp}$ as a variation of tilt, twist, and $n$ : (A) $\beta=50^{\circ}$, $\alpha$ varies and (B) $\alpha=30^{\circ}$ with varying $\beta$. 
The effect of $\beta$ is presented in Figure 4B: as $\beta$ increases, the magnitude of the saw-toothed oscillation decreases in conjunction with a decrease in $\mu_{\perp}$. The decrease in twist orients the methylene groups in such a way that dipole for an odd-valued chain has a higher dipole magnitude in the direction of the surface normal than an even-valued chain. Of the possibilities, this orientation counteracts the S-H dipole by the highest amount. In contrast, the methyl group for an even-chain structure has an orientation that minimizes the magnitude of its dipole with respect to the surface normal. In this way; the variation in angles between the methyl group dipole and the surface normal of odd vs. even chains cause the saw-toothed oscillation in $\mu_{\perp}$. At chain twists that approach $90^{\circ}$, the dipole orientation for odd and even chains become equivalent, eliminating the oscillation with $n$.

\section{Contact Angle}

By comparison of experimental contact angle values with previous observations of wettability for other surfaces, insights into the origins of adlayer properties can be made. For reference, bare gold substrates are wetted by water (i.e., $\theta=\sim 0^{\circ}$ ), ${ }^{70}$ hydrophobic surfaces such as paraffin and hexamethylethane have surfaces of predominately methyl groups and yield $\theta_{\mathrm{a}}$ values of $\sim 130^{\circ},{ }^{18}$ whereas surfaces with methylene groups, as with polyethylene, exhibit $\theta_{\mathrm{a}} \sim 102^{\circ} .{ }^{71}$ The most hydrophobic hexanethiolate monolayer we have observed (prepared using a $>24 \mathrm{~h}$ immersion in $1.0 \mathrm{mM}$ hexanethiol) exhibited a $\theta_{\mathrm{a}}$ value of $114^{\circ}$.

A plot of the advancing contact angle $\left(\theta_{a}\right)$ for water at the series of $n$-alkanethiolate monolayers $(n=4-11,13-15,17)$ is shown in Figure 5 . These results yield several important observations. As $n$ increases, the contact angle reaches a limiting value. The limiting value for $\theta_{\mathrm{a}}\left(\sim 115^{\circ}\right)$ is comparable to those for interfaces terminated by $-\mathrm{CH}_{3}$ groups (e.g., paraffin), ${ }^{18}$ suggesting that the hydrocarbon chains approach, as expected, a densely packed structure. However, when $n<\sim 8$, there is a decrease in $\theta_{\mathrm{a}}$ with chain length. There also exists a clear odd-even oscillation in contact angle. These trends are strikingly similar to those 
exhibited by the modeled dipole moment for an $n$-alkanethiolate monolayer of the conformation $\alpha=30^{\circ}$ and $\beta=50^{\circ}$, which is also presented in Figure 5 (squares). Since the dipole moment of the adlayer directly affects the resulting contact angle, ${ }^{58}$ a discussion of the relationship of the trends in the experimental and model data follows.

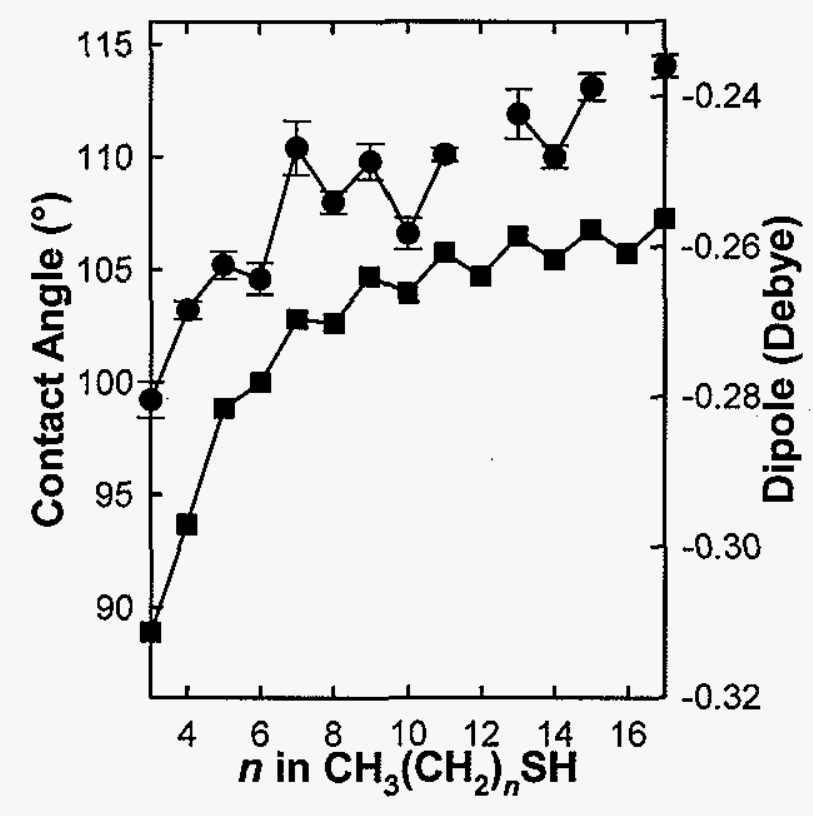

Figure 5. Experimental contact angle ( $\theta_{\mathrm{a}}$, circles) of $n$-alkanethiolate monolayers on gold, and modeled dipole (squares) of analogous thiols with $\alpha$ $=30^{\circ}$ and $\beta=50^{\circ}$. The length of the error bars represent two standard deviations of 5 measurements.

The contact angles show a dramatic decrease at short chain lengths. The decrease indicates that when $n<8$, the layer is more hydrophilic. This trend also appears in earlier work. $^{18,20-23,48}$ Interestingly, the evolution of dipole moment follows that of the contact angle data, which suggests the decrease in contact angle is not merely a manifestation of a decrease in packing density as previously suggested, but is also affected by the increase in $\mu_{\perp}$.

The contact angle data also display an odd-even effect, as previously noted for alkanethiols $^{53,72}$ and alkanoic acids ${ }^{73}$ on silver and methoxy-terminated adlayers on gold. ${ }^{54}$ Trends such as this have been observed using hexadecane, a low surface tension liquid. ${ }^{58}$ To our knowledge, this oscillation is the first reported with water, a high surface tension liquid. 
Although close to the precision of these measurements, the data presented in Figure 5 show a clear odd-even trend. In addition, the calculated dipole data exhibit the same odd-even variation in magnitude, suggesting the adlayer dipole is directly affecting the observed contact angle. We rationalize the decrease in observed contact angle for even-valued chains by discussion of $\mu_{\perp}$. The increased $\mu_{\perp}$ creates a higher surface normal electric field, and consequently an increase in the dipole-dipole interactions between the surface and contacting probe liquid. ${ }^{58}$ This phenomenon results in a decrease in the observed contact angle.

\section{Ellipsometry}

Ellipsometric measurements have been widely utilized to estimate the thickness of these adlayer systems. ${ }^{1,3,17-21}$ For each chain length in this study, three substrates were prepared and interrogated by ellipsometry, with $\Delta$ and $\Psi$ values analyzed using a three-phase, parallel-layer model and a refractive index of 1.50 for the adlayer (see below for a discussion of the impact of the value of refractive index). These results are presented in Figure 6 (circles), and show a linear increase in film thickness with increasing chain length. In addition, the data hint toward an odd-even chain length dependence. For comparison, the

model data for the same approximate conformation $\left(\alpha=30^{\circ} \text { and } \beta=50^{\circ}\right)^{3,6,19,20,27,46}$ are also shown in Figure 6 (triangles).

First, a comparison of the slope of experimental ellipsometric thickness values $\left(1.14 \AA n^{-1}\right)$ with the slope of the model data $\left(1.09 \AA n^{-1}\right)$ shows a small difference, approximately $5 \%$. In addition, the experimental data hint to a small degree at an odd-even oscillation in thickness as indicated by the modeling data. The suggestion of this trend is primarily based on the average of the measured film thicknesses from $n=4-10$. However, statistical analysis does not support reaching such a conclusion; the intercepts of the linear regression for odd and even chains were not significantly different when associated with the experimental error. The expected thickness increase $(\sim 0.8 \AA$ when increasing $n$ from an odd- 
to and even-value, as opposed to $\sim 1.4 \AA$ when adding a methylene unit to an even-chain) approaches the experimental precision limit $(\sim 2 \AA)$. However, the possibility of the oscillation follows that predicted by the model.

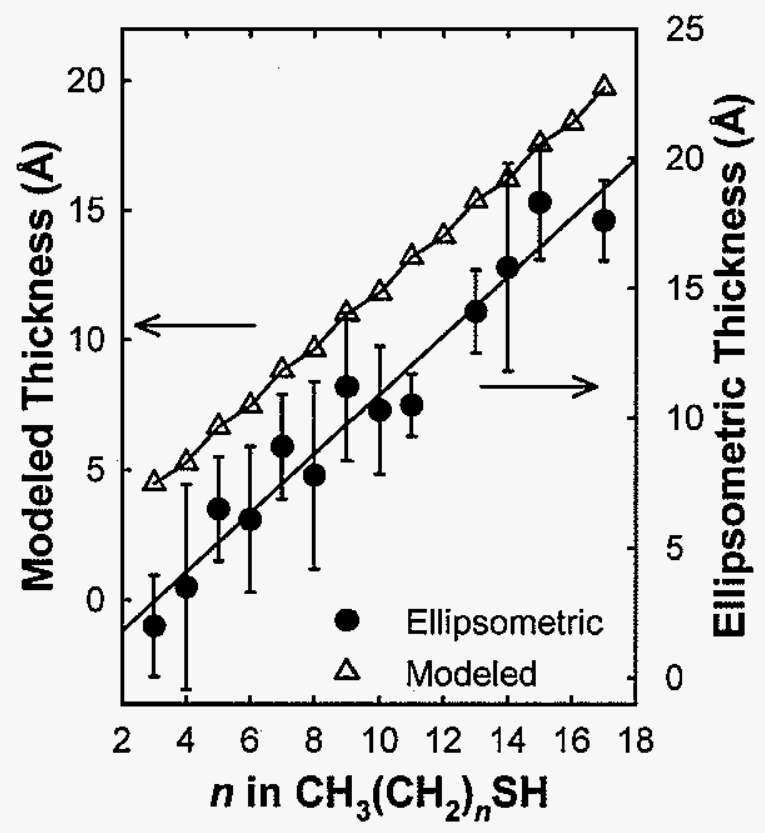

Figure 6. Ellipsometric thickness (circles) of $n$-alkanethiolate monolayers on gold. The length of the error bars represent two standard deviations of 15 measurements. The best fit line gives a slope of $1.14 \AA n^{-1}$ and intercept of $-0.46 \AA$. For comparison, modeled thickness (triangles) of the same adlayer is presented $\left(\alpha=30^{\circ}\right.$ and $\left.\beta=50^{\circ}\right)$.

These observations have led to a critical reexamination of the sources of uncertainty in ellipsometric measurements. The experimental error typically exhibited by ellipsometric measurements of adlayer systems is a combination of the uncertainties in the adlayer refractive index and in the optical function of the underlying gold substrate. The refractive index for these adlayers is expected to be in the region of $1.45-1.500^{3,17,18,21}$ When the experimental data were treated by the three-phase, parallel-layer model with the two extremes in refractive index, the thickness varied for each chain length. However, a maximum change in thickness of less than $2 \AA$ was observed. This difference is the same as the expected error accompanied by the uncertainty in refractive index of $0.05 .{ }^{17}$ The slopes of resulting 
thickness vs. $n$ plots were 1.22 and $1.14 \AA n^{-1}$ for a refractive index of 1.45 and 1.50 , respectively. In addition to the uncertainty in the overall refractive index, it may also vary with chain length, as a function of whether $n$ is odd or even, or as a function of adlayer orientation. $^{3}$

Another source of experimental error can lead to uncertainties in the value of the intercept of a plot of thickness vs. $n$. One such error is the determination of the optical constant of the underlying gold substrate. That is, the chemisorption of the sulfur head group may affect the dielectric constant of the underlying gold, ${ }^{3}$ which can be manifested in the measured thickness. This effect would be coverage dependant; however, we believe the coverage of our adlayers to be effectively constant for all values of $n$. Therefore, only a concurrent increase or decrease in thickness for all chain lengths is expected. The uncertainty associated with determination of the optical constants of the substrate is implicated as a possible cause for the negative intercept exhibited by the experimental thickness values.

\section{$\underline{\text { Conclusions }}$}

A number of examples of odd-even chain length dependencies of $n$-alkanethiolate monolayers have been observed. ${ }^{20,29,30,49-56} \mathrm{Up}$ to this point, proposed explanations for these trends have been generally discussed, but not rigorously examined. As an extension of these discussions, we have proposed molecular modeling as a facile means to explore the adlayer properties on a fundamental level. For example, we have utilized calculations to predict trends in contact angle and thickness of thiolate adlayers. In the course of these calculations, we have forecasted odd-even oscillations in both properties, which were observed to some degree in both sets of experimental measurements.

The molecular dipole moment $\left(\mu_{\perp}\right)$ as assessed by modeling calculations is directly compared to the observed trends in measured contact angle. Upon examination of these data, two important discoveries are apparent. First, $\mu_{\perp}$ exhibits an odd-even effect with respect to 
$n$. Second, an increase in $\mu_{\perp}$ at chain lengths shorter than $n=8$ is observed. In their entirety, both observations are translated into trends in the contact angle values using water as the probe liquid. The odd-even oscillation in wettability can be fully described by the oscillation in $\mu_{\perp}$, and the decrease in contact angle value is driven by the increase in $\mu_{\perp}$. Therefore, we suggest that the molecular dipole moment of the adlayer is directly affecting the observed wettability of the interface.

The modeling data also establishes the expected thickness of the adlayers as a function of orientation and $n$. Comparison of the slope of the thickness vs. chain length for the experimental and modeling data, correspond well for an $\alpha=\sim 30^{\circ}$, with a discrepancy of approximately $5 \%$. The model data also presents a slight odd-even oscillation in thickness, which has not previously been suggested for this system. The modeling data also shows that this effect is very dependant upon adlayer component orientation. In the experimental data, the odd-even effect is below the limit of experimental error, however a hint of the trend is observed.

In summary, this manuscript has shown that modeling calculations can be utilized to predict interfacial properties of adlayer systems, and provide fundamental explanations for observed trends in these properties. In addition, we present the odd-even oscillation with chain length of two properties, thickness and contact angle, for $n$-alkanethiolates on gold that have not previously been observed.

\section{Acknowledgments}

This work was supported by the Basic Energy Sciences program of the U.S. Department of Energy. G.A.E. gratefully acknowledges a Conoco-Phillips graduate research fellowship. Valuable discussions with M. Gordon, I. Adamovic, and D. Zorn are also acknowledged. The authors would like to thank Professor Andrew Hillier at Iowa State University for the use of the ellipsometer and helpful discussions. The Ames Laboratory is 
operated by Iowa State University for the U.S. Department of Energy under Contract W-7405-ENG-82.

\section{$\underline{\text { References }}$}

(1) Nuzzo, R. G.; Allara, D. L. J. Am. Chem. Soc. 1983, 105, 4481-3.

(2) Li, T. T.-T.; Weaver, M. J. J. Am. Chem. Soc. 1984, 106, 6107-8.

(3) Porter, M. D.; Bright, T. B.; Allara, D. L.; Chidsey, C. E. D. J. Am. Chem. Soc. 1987, 109, 3559-68.

(4) Nuzzo, R. G.; Zegarski, B. R.; Dubois, L. H. J. Am. Chem. Soc. 1987, 109, 733-40.

(5) Ulman, A. An Introduction to Ultrathin Organic Films from Langmuir-Blodgett to Self-Assembly; Academic Press: San Diego, CA, 1991.

(6) Dubois, L. H.; Nuzzo, R. G. Annu. Rev. Phys. Chem. 1992, 43, 437-63.

(7) Love, J. C.; Estroff, L. A.; Kriebel, J. K.; Nuzzo, R. G.; Whitesides, G. M. Chem. Rev. 2005, 105, 1103-69.

(8) Chidsey, C. E. D.; Liu, G.-Y.; Rowntree, P. A.; Scoles, G. J. Chem. Phys. 1989, 91, 4421-3.

(9) Chidsey, C. E. D.; Loiacono, D. N. Langmuir 1990, 6, 682-91.

(10) Camillone, N., III; Chidsey, C. E. D.; Liu, G.-Y.; Putvinski, T. M.; Scoles, G. J. Chem. Phys. 1991, 94, 8493-502.

(11) Camillone, N., III; Chidsey, C. E. D.; Liu, G.-Y.; Scoles, G. J. Chem. Phys. 1993, 98, 3503-11.

(12) Dubois, L. H.; Zegarski, B. R.; Nuzzo, R. G. J. Chem. Phys. 1993, 98, 678-88.

(13) Camillone, N., III; Chidsey, C. E. D.; Liu, G.-Y.; Scoles, G. J. Chem. Phys. 1993, 98 , 4234-45.

(14) Fenter, P.; Eberhardt, A.; Eisenberger, P. Science 1994, 266, 1216-8.

(15) Camillone, N., III; Eisenberger, P.; Leung, T. Y. B.; Schwartz, P.; Scoles, G.; Poirier, G. E.; Tarlov, M. J. J. Chem. Phys. 1994, 101, 11031-6.

(16) Camillone, N., III; Leung, T. Y. B.; Schwartz, P.; Eisenberger, P.; Scoles, G. Langmuir 1996, 12, 2737-46.

(17) Nuzzo, R. G.; Fusco, F. A.; Allara, D. L. J. Am. Chem. Soc. 1987, 109, 2358-68. 
(18) Bain, C. D.; Troughton, E. B.; Tao, Y.-T.; Evall, J.; Whitesides, G. M.; Nuzzo, R. G. J. Am. Chem. Soc. 1989, 111, 321-35.

(19) Nuzzo, R. G.; Dubois, L. H.; Allara, D. L. J. Am. Chem. Soc. 1990, 112, 558-69.

(20) Laibinis, P. E.; Whitesides, G. M.; Allara, D. L.; Tao, Y.-T.; Parikh, A. N.; Nuzzo, R. G. J. Am. Chem. Soc. 1991, 113, 7152-67.

(21) Biebuyck, H. A.; Bain, C. D.; Whitesides, G. M. Langmuir 1994, 10, 1825-31.

(22) Bain, C. D.; Whitesides, G. M. J. Am. Chem. Soc. 1988, 110, 5897-8.

(23) Bain, C. D.; Biebuyck, H. A.; Whitesides, G. M. Langmuir 1989, 5, 723-7.

(24) Whitesides, G. M.; Laibinis, P. E. Langmuir 1990, 6, 87-96.

(25) Dubois, L. H.; Zegarski, B. R.; Nuzzo, R. G. J. Am. Chem. Soc. 1990, 112, 570-9.

(26) Bain, C. D.; Whitesides, G. M. J. Am. Chem. Soc. 1988, 110, 6560-1.

(27) Nuzzo, R. G.; Korenic, E. M.; Dubois, L. H. J. Chem. Phys. 1990, 93, 767-73.

(28) Truong, K. D.; Rowntree, P. A. J. Phys. Chem. 1996, 100, 19917-26.

(29) Bryant, M. A.; Pemberton, J. E. J. Am. Chem. Soc. 1991, 113, 8284-93.

(30) Bryant, M. A.; Pemberton, J. E. J. Am. Chem. Soc. 1991, 113, 3629-37.

(31) Widrig, C. A.; Alves, C. A.; Porter, M. D. J. Am. Chem. Soc. 1991, 113, 2805-10.

(32) Alves, C. A.; Smith, E. L.; Porter, M. D. J. Am. Chem. Soc. 1992, 114, 1222-7.

(33) McCarley, R. L.; Kim, Y.-T.; Bard, A. J. J. Phys. Chem. 1993, 97, 211-5.

(34) McCarley, R. L.; Dunaway, D. J.; Willicut, R. J. Langmuir 1993, 9, 2775-7.

(35) Liu, G.-Y.; Salmeron, M. B. Langmuir 1994, 10, 367-70.

(36) Poirier, G. E.; Tarlov, M. J.; Rushmeier, H. E. Langmuir 1994, 10, 3383-6.

(37) Schönenberger, C.; Jorritsma, J.; Sondag-Huethorst, J. A. M.; Fokkink, L. G. J. J. Phys. Chem. 1995, 99, 3259-71.

(38) McDermott, C. A.; McDermott, M. T.; Green, J.-B.; Porter, M. D. J. Phys. Chem. $1995,99,13257-67$.

(39) Paradis, E.; Rowntree, P. J. Electroanal. Chem. 2003, 550-551, 175-85.

(40) Strong, L.; Whitesides, G. M. Langmuir 1988, 4, 546-58.

(41) Sellers, H.; Ulman, A.; Shnidman, Y.; Eilers, J. E. J. Am. Chem. Soc. 1993, 115 , 9389-401. 
(42) Zhang, H.; Chung, S.-W.; Mirkin, C. A. Nano Lett. 2003, 3, 43-5.

(43) . Beardmore, K. M.; Kress, J. D.; Grønbech-Jensen, N.; Bishop, A. P. Chem. Phys. Lett. 1998, 286, 40-5.

(44) Lay, M. D.; Varazo, K.; Stickney, J. T. Langmuir 2003, 19, 8416-27.

(45) Widrig, C. A.; Chung, C.; Porter, M. D. J. Electroanal. Chem. 1991, 310, 335-59.

(46) Parikh, A. N.; Allara, D. L. J. Chem. Phys. 1992, 96, 927-45.

(47) Rong, H.-T.; Frey, S.; Yang, Y.-J.; Zharnikov, M.; Buck, M.; Wühn, M.; Wöll, C.; Helmchen, G. Langmuir 2001, 17, 1582-93.

(48) Shafrin, E. G.; Zisman, W. A. J. Phys. Chem. 1962, 66, 740-8.

(49) Day, B. S.; Morris, J. R. J. Phys. Chem. B 2003, 107, 7120-5.

(50) Kim, H. I.; Houston, J. E. J. Am. Chem. Soc. 2000, 122, 12045-6.

(51) Smalley, J. F.; Feldberg, S. W.; Chidsey, C. E. D.; Linford, M. R.; Newton, M. D.; Liu, Y.-P. J. Phys. Chem. 1995, 99, 13141-9.

(52) Sumner, J. J.; Weber, K. S.; Hockett, L. A.; Creager, S. E. J. Phys. Chem. B 2000, 104, 7449-54.

(53) Walczak, M. M.; Chung, C.; Stole, S. M.; Widrig, C. A.; Porter, M. D. J. Am. Chem. Soc. 1991, 113, 2370-8.

(54) Wenzl, I.; Yam, C. M.; Barriet, D.; Lee, T. R. Langmuir 2003, 19, 10217-24.

(55) Wong, S.-S.; Takano, H.; Porter, M. D. Anal. Chem. 1998, 70, 5209-12.

(56) Alloway, D. M.; Hofmann, M.; Smith, D. L.; Gruhn, N. E.; Graham, A. L.; Colorado, R., Jr; Wysocki, V. H.; Lee, T. R.; Lee, P. A.; Armstrong, N. R. J. Phys. Chem. B 2003, 107, 11690-9.

(57) Evans, S. D.; Ulman, A. Chem. Phys. Lett. 1990, 170, 462-6.

(58) Colorado, R., Jr; Lee, T. R. J. Phys. Org. Chem 2000, 13, 796-807.

(59) Liu, Y.-P.; Newton, M. D. J. Phys. Chem. 1994, 98, 7162-9.

(60) Zhong, C.-J.; Brush, R. C.; Anderegg, J.; Porter, M. D. Langmuir 1999, 15, 518-25.

(61) Rodriguez, J. F.; Mebrahtu, T.; Soriaga, M. P. J. Electroanal. Chem. 1987, 233, 2839.

(62) Walczak, M. M.; Popenoe, D. D.; Deinnhammer, R. S.; Lamp, B. D.; Chung, C.; Porter, M. D. Langmuir 1991, 7, 2687-93.

(63) Hamelin, A. J. Electroanal. Chem. 1984, 165, 167-80. 
(64) Hamelin, A.; Lipkowski, J. J. Electroanal. Chem. 1984, 171, 317-30.

(65) Allara, D. L.; Nuzzo, R. G. Langmuir 1985, 1, 45-52.

(66) Chem3D, Ultra 8.0, CambridgeSoft: Cambridge MA, 2004.

(67) Majumder, C.; Briere, T. M.; Mizuseki, H.; Kawazoe, Y. J. Chem. Phys. 2002, 117, 2819-22.

(68) Anderson, J. E.; Slichter, W. P. J. Phys. Chem. 1965, 69, 3099-104.

(69) CRC Handbook of Chemistry and Physics; Lide, D. R., Ed.; CRC Press: Boca Raton Fl, 1995.

(70) Israelachvili, J. N. Intermolecular and Surface Forces; 2nd ed.; Academic Press: San Diego, CA, 1991.

(71) Holmes-Farley, S. R.; Reamey, R. H.; McCarthy, T. J.; Deutch, J.; Whitesides, G. M. Langmuir 1985, 1, 725-40.

(72) Chang, S.-C.; Chao, I.; Tao, Y.-T. J. Am. Chem. Soc. 1994, 116, 6792-805.

(73) Tao, Y.-T. J. Am. Chem. Soc. 1993, 115, 4350-8. 


\title{
CHAPTER 4: MASS TRANSPORT EFFECTS ON THE FORMATION OF SELF-ASSEMBLED MONOLAYERS
}

\author{
Grant A. Edwards, Adam Johan Bergren, Erik J. Cox, and Marc D. Porter
}

\begin{abstract}
Ames Laboratory-U.S.D.O.E., Department of Chemistry, and the Institute for Combinatorial Chemistry, Iowa State University, Ames, Iowa 50011
\end{abstract}

\begin{abstract}
$\underline{\text { Abstract }}$
In this work, equations that relate the impingement of thiol molecules onto a gold surface are derived for two substrate/deposition vessel geometries. The first model results in primarily linear diffusion, whereas hemispherical diffusion is important in the other. The relationship between thiol concentration, immersion time, and thiol impingement is shown to depend on deposition geometry. With these predictive equations as guides, adlayer formation is followed using infrared reflection-absorption spectroscopy, contact angle, and heterogeneous electron transfer kinetics. This work shows that the mass transport of thiol molecules to the gold surface must be carefully considered and controlled in order to create reproducible adlayers: both total impingement and the rate of impingement play major roles in determining the structure and function of the adlayer. This dependence is attributed to variation in the nucleation and growth conditions.
\end{abstract}

\section{$\underline{\text { Introduction }}$}

Self-assembled monolayers (SAMs) offer a highly effective strategy for the systematic manipulation of interfacial properties. ${ }^{1-7}$ Among many possible systems, "thiols on gold" have attracted considerable attention, owing to their relative ease of preparation, 
stability, and structural definition. ${ }^{8-12}$ The average $e^{8,10,12-15}$ and localized ${ }^{16-18}$ structure of alkanethiolate monolayers have been extensively characterized. A survey of recent literature shows a wide variability in the conditions used to form these monolayers for both applied ${ }^{19-23}$ and fundamental ${ }^{24-35}$ studies. For example, Truong and Rowntree ${ }^{35,36}$ utilized thiol concentrations between 0.2 and $2 \mu \mathrm{M}$ and deposition times between $1 \mathrm{~min}$ and several days to investigate absorption kinetics. In contrast, concentrations as high as $30 \mathrm{mM}$ and deposition times up to $24 \mathrm{~h}$ have been used. ${ }^{33,34}$ In addition to immersion time and thiol concentration, surface crystallinity $y^{37,38}$ and roughness ${ }^{39}$ are known to affect the resulting characteristics of the adlayer.

It is widely acknowledged that the observed interfacial properties and structure of an adlayer formed under seemingly similar conditions in different laboratories may vary, even though there is not an immediately apparent origin for the difference. ${ }^{40}$ In addition, it can prove challenging to obtain SAMs with identical function in an electrochemistry experiment although ostensibly identical preparation conditions may have been used. Other measures of adlayer performance have also suffered from the same reproducibility challenges. In any case, it is generally important to develop procedural protocols that yield reproducible performance with respect to a specific application. To effectively manipulate the adlayer reproducibility, the factors that control film properties, both in their final form and as they evolve during assembly, are important. Many of these properties may be dependent on a wide range of conditions, some of which may be easily controlled and others that possible will prove more difficult to manage with high precision.

The assembly mechanism of alkanethiols and related compounds onto gold has been investigated using a wide range of surface analysis tools. ${ }^{6,11,12,16,18,24,41-75}$ The combined weight of these investigations has qualitatively characterized the absorption process by four distinct stages. ${ }^{41}$ In Stage 1, the molecules on the surface can be described as a two-dimensional lattice-gas during the initial stage of formation. These highly mobile 
molecules are oriented with their alkyl chain parallel to the surface and are present at a relatively low level. At this point, agostic bonds ${ }^{76}$ and solvophobic interactions appear to drive adsorption. The beginning of Stage 2 can be identified by the nucleation of small, ordered molecular islands induced by van der Waals interactions between neighboring adsorbates as coverage increases. As imaged by STM, ${ }^{16}$ the surface concentration of adsorbate is only a fraction of the packing limit value, with estimates of packing density resulting in an area per adsorbate of $60 \AA^{2}$ (a coverage of $2.3 \times 10^{-10} \mathrm{~mol} \mathrm{~cm}^{-2}$ ) for $\mathrm{HS}\left(\mathrm{CH}_{2}\right)_{6} \mathrm{OH}$.

In Stage 3, the molecular axis of the alkyl chain begins to reorient toward the surface normal, creating islands of adsorbates in a "standing-up" configuration. This change also yields vacancies that are filled in by additional precursors. As Stage 3 progresses, the final stage initiates the healing of defects within the adlayer, which occurs over a longer time scale. This healing can comprise, for example, gauche to trans conformational transitions of the chains and further filling of adsorbate vacancies. These final stages are also driven by van der Waals interactions between alkyl chains of neighboring adsorbates, and by movement of the system toward a low surface free energy with respect to solvent-film interactions. The culmination of these forces orients the adsorbate more normal to the surface, allowing the surface concentration to increase to a closest-packed value ( $22 \AA$ per molecule or $7.6 \times 10^{-10} \mathrm{~mol} \mathrm{~cm}{ }^{-2}$ ). The combination of head-group binding and intermolecular forces leads to an "equilibrium" structure that is characterized by a high packing density (formation of a full monolayer), strong molecular ordering (presumed all-trans zigzag conformational sequences), and chemical and mechanical stability.

As is apparent, the complex assembly mechanism of this system places a premium on the ability to trace structural details back to what can be subtle differences in preparative procedures. The challenge in making these important connections is further complicated by the widespread use of various methodologies which interrogate different structural features 
and/or interfacial properties of the adlayer. For example, while one procedure may yield a reproducible surface for use in the preparation of an immunological capture surface, ${ }^{77}$ the same protocol may result in a coating with an unacceptably large variation in blocking characteristics for fundamental studies of heterogeneous electron transfer. For example, Ma and Lennox ${ }^{40}$ discuss these reproducibility problems, and found that by controlling the applied potential during the deposition of hexadecanethiol onto gold, a monolayer with strong blocking characteristics can be achieved. These and other examples of limited reproducibility point to the need to gain a more in-depth understanding of the effects of the deposition conditions and the roughness, crystallinity, and cleanliness of the underlying substrate.

In this work, we extend earlier investigations on the role of mass transfer with respect to adlayer deposition. ${ }^{41,55,56}$ We take, however, a slightly different tactic by examination of how the geometry (i.e., size) of the substrate and reaction vessel influence the structure, wetting, and electrochemical properties of the resulting adlayer. To accomplish this goal, we also make every attempt to maintain the surface structure of our gold substrate constant through pretreatment procedures that have a high level of reproducibility. Therefore, a theoretical model is developed that relates the impingement of thiols from solution onto the gold substrate to a geometry-dependant time parameter. The model considers the thiol solution concentration, the size and shape of the substrate, and the size and shape of the deposition container, all of which couple to define whether the delivery of the reactant is controlled by linear or radial diffusion, or a confluence of both.

With these predictive equations as guidelines, adlayer formation is followed using infrared reflection-absorption spectroscopy (IRRAS), contact angle, and the heterogeneous electron-transfer kinetics of $\mathrm{Fe}\left(\mathrm{CN}_{6}\right)^{4-13-}$. These experimental techniques are complementary in nature, each interrogating different aspects of the adlayer structure or function. Our results are discussed in relation to how total impingement and impingement rate affect adlayer characteristics. This work demonstrates that mass transport of thiol molecules to the gold 
surface must be carefully considered and controlled to reproduce the nucleation and growth conditions.

\section{Mass Transfer Model}

Previous works considered the role of thiol concentration in attempts to relate diffusion to adlayer deposition. Karpovich and Blanchard ${ }^{47}$ followed the concentration dependence of monolayer deposition during a quartz crystal microbalance investigation of formation kinetics. Jung and Campbel1 ${ }^{55,56}$ utilized Fick's Law to calculate concentration profiles during adlayer deposition in order to determine the sticking coefficient for thiols on gold. Camillone $e^{41}$ derived a linear diffusion model as a means to calculate the time required for the impingement of one-half of a monolayer from solution. A similar approach is taken herein; however, we utilize a three-dimensional random-walk model in stagnant solution, while extending the model to include considerations of the volume of solution and the geometry of the assembly vessel.

There are several simplifying assumptions needed in order to use the random-walk model. First, only diffusion and not convection (e.g., density gradients or stirring) is responsible for the mass transport of molecules to the surface. In addition, the microscopic substrate topography is not considered in the model derivation. The mechanism for thiol adsorption is also neglected. In essence, this model simply determines the number of molecules that have an opportunity to interact with the surface (i.e., impinge) as a function of time, concentration, and reaction vessel geometry.

Two limiting cases for the diffusional impingement of thiols onto gold surfaces are examined. The first case considers only linear diffusion as dictated by the size of the substrate with respect to the dimensions of the reaction vessel. Figure 1A serves as an illustrative example. This setup resembles the approach typically utilized to form a monolayer on a large substrate, e.g., a gold-coated microscope slide used in IRRAS characterizations. The second 
case reflects a design in which radial diffusion can play a role in the delivery of the reactant to the electrode. It reflects the situation in which the gold substrate is much smaller than any of the dimensions of the solution-containing vessel. This configuration mimics that often employed in the modification of much smaller substrates, like those used in electrochemical experiments.
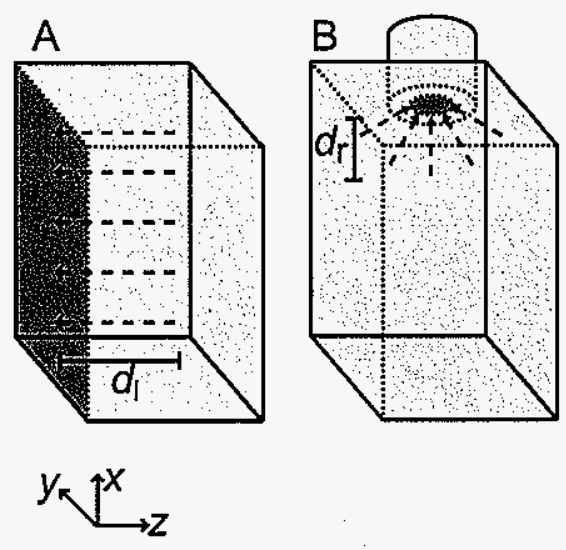

Figure 1. Two deposition geometries considered in the impingement model. Edges of the light grey box depict the edges of the deposition container, while the dark grey represents the gold substrate. (A) Linear model where the substrate and deposition container are of the same dimensions. (B) Radial diffusion can become important when the deposition container is much larger than the substrate.

As a starting point: variables employed in both situations are defined: $C=$ thiol concentration in bulk solution; $\Gamma_{0}=$ limiting surface concentration for a thiolate monolayer on an atomically smooth $\mathrm{Au}(111)$ surface $\left(7.6 \times 10^{-10}\right.$ moles $\left.\mathrm{cm}^{-2}\right) ;{ }^{78} A=$ surface area of the $\mathrm{Au}$; and $D=$ diffusion coefficient of the thiol molecule in ethanol $\left(5.0 \times 10^{-6} \mathrm{~cm}^{2} \mathrm{~s}^{-1}\right) \cdot{ }^{41,55} \mathrm{To}$ generalize the model, a fractional factor $\chi$ is introduced that represents the number of monolayers that would form solely based on impingement onto the surface, (e.g., a $\chi$ of 0.5 means that $3.80 \times 10^{-10}$ moles $\mathrm{cm}^{-2}$ of thiol have impinged upon the surface). Therefore, the volume element $(V)$ of a homogenous solution that contains the equivalent number of thiols can be written as 


$$
V=\frac{\chi \Gamma_{0} A}{C}
$$

which creates a reaction vessel with arbitrary dimensions. It is however, the relative size and shape of the reaction vessel with respect to the substrate that dictate the actual shape of the volume element and therefore the mode of diffusional mass transfer. The remainder of this section details the development of the two models and then examines the implications of their differences on adlayer formation.

\section{Linear Diffusion ModeI}

The model geometry depicted in Figure.1A assumes that linear diffusion strongly dominates the delivery of thiols to the substrate. This model geometry is reminiscent of that used to form monolayers on a gold-coated glass slide by using a microscope slide in a staining jar. Staining jars, like those from most supply houses, have volumes of $\sim 75 \mathrm{~mL}$, and internal dimensions of $7.6 \times 2.5 \times 2.5 \mathrm{~cm}$. Therefore, an immersed glass microscope slide, which has a rectangular shape of roughly $7.6 \times 2.5 \mathrm{~cm}$, leaves little room for diffusion of thiols from beyond the edges of the slide (i.e., there is no contribution from nonlinear diffusion). This construct leads to an artificial volume element with two dimensions dictated by the surface of the substrate. The third dimension is defined by the thickness $\left(d_{\mathrm{L}}\right)$ of the solution required to yield a volume that contains the appropriate number of adsorbate precursors as required by the value of $\chi$. The value for $V$ in this case can be expressed as

$$
V=A d_{\mathrm{L}}
$$

By equating Equations 2 and 3 and solving for $d_{\mathrm{L}}$, we get

$$
d_{\mathrm{L}}=\frac{\chi \Gamma_{0}}{C}
$$

This equation therefore indicates that as long as $d_{\mathrm{L}}$ is less than the third dimension of the deposition container, the number of thiols present in the volume element is sufficient to satisfy that required by $\chi$. 
The second part of the model considers the role of diffusional mass transport. The time required $(t)$ for a molecule to become displaced a distance $x$ through Brownian motion ${ }^{79}$ is given by

$$
x=\sqrt{6 D t}
$$

If we then equate $d_{\mathrm{L}}$ in Equation 4 to $x$ in Equation 5 , the time $\left(t_{\mathrm{L}}\right)$ required for the transport of molecules from the entire volume element to the substrate via linear diffusion can be calculated as

$$
t_{\mathrm{L}}=\frac{\chi^{2} \Gamma_{0}^{2}}{6 D C^{2}}
$$

Equation 6 predicts that it would require $\sim 320 \mathrm{~min}$ for one monolayer of thiols $(\chi=1)$ to impinge the substrate at a solution concentration of $1.0 \mu \mathrm{M}$, and that the reaction vessel must support a solution layer thickness of at least $0.76 \mathrm{~cm}$. In addition, a slide immersed in a $1.0 \mathrm{mM}$ solution for $24 \mathrm{~h}$ equates to a $\chi$-value of 2118 .

\section{Radial Diffusion Model}

For the construct depicted in Figure 1B, the lateral dimensions of the substrate are much smaller than those of the reaction vessel. This approach to adlayer formation can therefore have a strong mass transport contribution from radial diffusion. Under these conditions, the parameter $d_{\mathrm{R}}$ carves out an artificial hemispherical container such that

$$
V=\frac{2 \pi d_{\mathrm{R}}^{3}}{3}
$$

If $d_{\mathrm{R}}$ is then connected to the volume of solution required to satisfy $\chi$, upon equating Equations 2 and 7 we can write

$$
d_{\mathrm{R}}=\sqrt[3]{\frac{3 \chi \Gamma_{0} A}{2 \pi C}}
$$


Finally, by applying the same three-dimensional random-walk model in Equation 5 to Equation 8 , the radial diffusion time parameter $\left(t_{\mathrm{R}}\right)$ is given by

$$
t_{\mathrm{R}}=\frac{1}{6 D}\left(\frac{3 \chi \Gamma_{0} A}{2 \pi C}\right)^{2 / 3}
$$

Equation 6 is rigorously valid only when radial diffusion controls the delivery of thiols to the gold surface. In practice, the radial model is inappropriate for small values of $d_{\mathrm{R}}$ since a hemispherical diffusion layer has not fully developed. While somewhat arbitrary, we have chosen a lower limit for $d_{\mathrm{R}}$ of at least five times the electrode radius $\left(r_{\mathrm{e}}\right)$ but smaller than the deposition container. This conservative estimate, we believe, will ensure radial diffusion is the dominant form of mass transfer of thiols to the substrate surface and leads to accurate calculation of impingement. As an example, the gold electrodes $\left(r_{\mathrm{e}}=0.080 \mathrm{~cm}\right)$ employed below require $d_{\mathrm{R}} \geq 0.40 \mathrm{~cm}$, which equates to a minimum immersion time of $90 \mathrm{~min}$.

Importantly, the same input parameters used for examples in the linear diffusion model (thiol concentration of $1.0 \mu \mathrm{M}$ and an area of $0.02 \mathrm{~cm}^{2}$, analogous to the polycrystalline gold electrodes) result in a much shorter time (21 $\mathrm{min})$ required to impinge this surface. These conditions require a deposition vessel with a radius of only $0.19 \mathrm{~cm}$ or larger.

\section{Model Comparison}

A few of the more informative predictions of the two models as a function of $C, \chi$, and $t$ are graphically illustrated in Figure 2 . The left column depicts the projections of the linear diffusion model, whereas those in the right column are for the radial diffusion model. All the results in Figure 2 were generated to closely match the experimental conditions used later in this manuscript; however, the resulting insights are more generally applicable. In addition, Table 1 presents the interrelationships of the important variables, which are 
cross-referenced to the plots of Figure 2. Before we begin an in-depth discussion of these relationships, note the lack of dependency on substrate area for the linear model, while the radial model exhibits a strong dependence on the area as a result of diffusion beyond the edges of the Au.

Table 1. The relationship between the deposition variables of the linear and radial models, as cross-referenced to the plots of Figure 2.

\begin{tabular}{lccc}
\hline \multicolumn{2}{c}{ Linear } & \multicolumn{2}{c}{ Radial } \\
\hline $\mathrm{A}:$ & $t_{\mathrm{L}} \propto C^{-2}$ & $\mathrm{~B}:$ & $t_{\mathrm{R}} \propto C^{-2 / 3}$ \\
$\mathrm{C}:$ & $\chi \propto C$ & $\mathrm{D}:$ & $\chi \propto C$ \\
$\mathrm{E}:$ & $\chi \propto t_{\mathrm{L}}^{1 / 2}$ & $\mathrm{~F}:$ & $\chi \propto t_{\mathrm{R}}^{3 / 2}$ \\
& & & $t_{\mathrm{R}} \propto A^{2 / 3}$ \\
\hline
\end{tabular}

Plots of $C$ as a function of $t$ for three $\chi$ values $(0.10,1.0$, and 10.) are shown in Figures $2 \mathrm{~A}$ and $2 \mathrm{~B}$ for the linear and radial models, respectively. As shown in Table $1, t_{\mathrm{L}}$ is proportional to $C^{-2}$, and $t_{\mathrm{R}}$ to $C^{-2 / 3}$. As expected, the concentration required to maintain a constant impingement increases as time is reduced. In addition, higher impingement values require larger thiol concentrations for both models. More importantly, the radial diffusion model requires a much smaller value of $C$ to reach a given impingement, as radial diffusion is more efficient at delivery of molecules to the substrate surface. This situation is illustrated by the difference in the scales for the $y$-axis of the two plots.

Next, Figures 2C and 2D show a linear relationship between $C$ and $\chi$, as outlined in Table 1. Overall, an increase in $C$ affects a concurrent increase in $\chi$. Again, there is a dramatic difference of impingement between the linear and radial mass transfer cases. The radial case is also more sensitive to the deposition time as reflected by the larger spread of the slopes for the three times (the proportionality constant for Equation D of Table 1 is large than that for Equation C). 

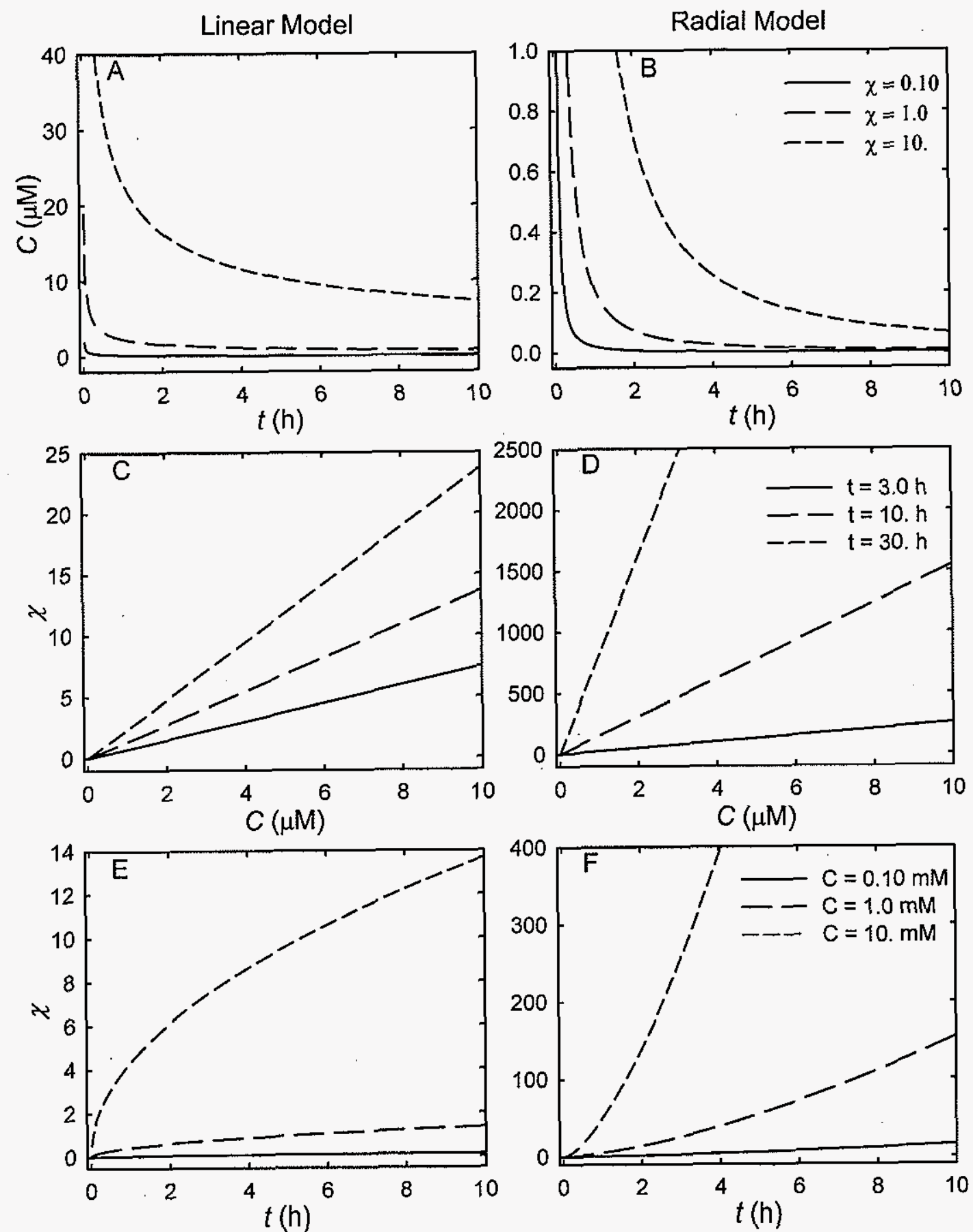

Figure 2. Comparison of linear (left column) and radial (right column) diffusion models. The calculations utilize these constants: $D=5.0 \times 10^{-6} \mathrm{~cm}^{2} \mathrm{~s}^{-1}, \Gamma_{0}=7.6 \times 10^{-10} \mathrm{~mol} \mathrm{~cm}{ }^{-2}, A=0.020 \mathrm{~cm}^{-2}$. Note: the radial model is used only for deposition times greater than $1.5 \mathrm{~h}$ $\left(r_{\mathrm{e}}=0.080 \mathrm{~cm}\right)$; due to radial diffusion requirements. 
A comparison of Figures $2 \mathrm{E}$ and $2 \mathrm{~F}$ also illustrate the difference between the linear and radial diffusional regimes for the time required to impinge the surface with an equal number of molecules. In a solution consisting of $1.0 \mu \mathrm{M}$ thiol, the linear case requires $53000 \mathrm{~h}$ to achieve an impingement of 100 , whereas the radial case only requires $7.5 \mathrm{~h}$.

The most striking difference between the linear and radial diffusion models is evident when examining Figures $2 \mathrm{E}$ and $2 \mathrm{~F}$. Again, the magnitude of $\chi$ is much larger for the radial model when all other variables are held constant. For the linear case, the rate of impingement (the instantaneous slope) decreases as the deposition time increases. Conversely, this rate increases for the radial case.

This analysis underscores the dramatic difference in impingement rates and numbers for different deposition geometries. That is, immersion of geometrically dissimilar substrates into a solution of the same thiol concentration and length of time can lead to a difference in nucleation and growth conditions.

At issue, then, is how impingement on two substrates by the same overall number of reactants, but with different rates, affects the structure and interfacial properties of the adlayer. Samples with a higher initial impingement rate may have a higher density of defects due to lattice mismatch at the boundary of different nucleation sites, ${ }^{80}$ noting that a high rate of initial impingement results in a larger number of nucleation sites. The overall impingement ratio, $\chi$, is also an important condition to specify when comparing adlayer properties. For example, interrogating a monolayer prepared with $\chi$ less than unity yields characteristics of an incomplete monolayer. However, a $\chi$ greater than unity does not ensure the presence of a fully formed monolayer because of the complexity and rate for each of the phases of adlayer formation. The following sections of this paper build on these insights by comparison of the structural and interfacial properties of monolayers prepared under seemingly identical conditions, which are in fact different because of the importance of geometric factors in dictating the dominate mass transfer mode of reactant impingement. 


\section{Experimental}

\section{Reagents and Chemicals}

Distilled water was further purified by deionization with a Millipore Milli-Q water system. Punctilious ethanol was purchased from Aaper. Hexanethiol, ferrocyanide, hydroxymethylferrocene, and sodium fluoride were obtained from Aldrich. Isopropanol was supplied by Fisher. All chemicals were used as received.

\section{Substrate Preparation}

Gold substrates for IRRAS and contact angle measurements was prepared by the resistive evaporation of $\sim 10 \mathrm{~nm}$ of a Cr adhesion layer (rate $0.1 \mathrm{~nm} \mathrm{~s}^{-1}$ ), followed by $\sim 300 \mathrm{~nm}$ of $\mathrm{Au}$ (rate $\left.0.2 \mathrm{~nm} \mathrm{~s}^{-1}\right)$ onto clean microscope slides $(7.6 \times 2.5 \mathrm{~cm}$, Fisher) in an Edwards $306 \mathrm{~A}$ turbo-pumped coating system at a pressure of $8 \times 10^{-7} \mathrm{mbar}$.

Polycrystalline gold disk electrodes from Bioanalytical Systems (area $0.020 \mathrm{~cm}^{2}$ ) and custom fabricated solid gold slabs $(2 \times 3 \times 0.75 \mathrm{~cm})$ were used for electrochemical measurements (electron transfer kinetics). These electrodes were pretreated prior to monolayer formation through the following procedures: 1) polished for $60 \mathrm{~s}$ in a figure-eight pattern on Buhler Microcloth with successively finer grades $(1.0,0.3,0.05 \mu \mathrm{m})$ of slurried alumina; 2) sonicated in isopropanol and water for $20 \mathrm{~min}$; and 3) cycled once between 0.0 and $+1.5 \mathrm{~V}\left(50 \mathrm{mV} \mathrm{s}^{-1}\right)$ in $1.0 \mathrm{M} \mathrm{H}_{2} \mathrm{SO}_{4}$. Both the shape of the voltammogram (the presence of only one oxide formation and stripping wave $)^{81}$ and the charge under the stripping wave served as metrics for reproducible surface preparation. Figure 3 presents an example of the stripping curves for five electrodes spanning the range of accepted surfaces. Only electrodes with a stripping charge of $1.44 \pm 0.79 \mathrm{mC} \mathrm{cm}^{-2}$ were qualified; these electrodes were rinsed with water and ethanol, and then immediately immersed in thiol solution. The range in the stripping charge denoted above was derived from a large sampling of polished electrodes. We have observed features (e.g., in the double-layer region of the stripping voltammograms) that 
are easily overlooked. These features were correlated with variations in the charge under the gold oxide stripping wave. Upon reducing the qualification limits to the values presented above, a balance between tight quality control and polishing throughput was achieved. We also found that these metrics served as a facile way to determine when the Microcloth polishing-pads required replacement (typically after polishing 3-5 electrodes). The combination of mechanical polishing, electrochemical pretreatment, and quality control metrics have resulted in acceptable reproducibility $( \pm 5-10 \%)$ when measuring heterogeneous electron-transfer rates.

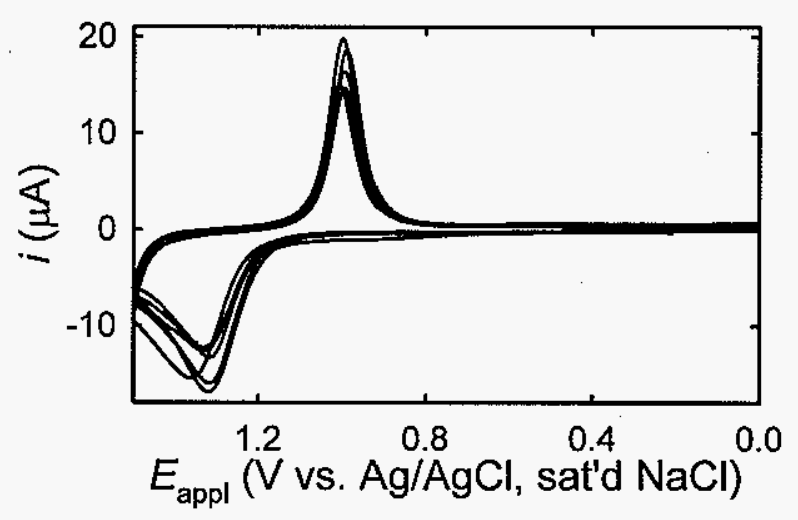

Figure 3. Electrochemical oxide formation and stripping voltammograms of 5 mechanically polished gold electrodes in $1.0 \mathrm{M} \mathrm{H}_{2} \mathrm{SO}_{4}$. Geometric area: $0.020 \mathrm{~cm}^{2}$, scan rate: $50 \mathrm{mV} \mathrm{s}^{-1}$.

Uncoated gold substrates were immersed, in the requisite deposition vessel, into an ethanolic thiol solution of prespecified concentration for a predefined length of time, rinsed copiously with ethanol, and dried in a directed stream of high purity nitrogen. Gold coated glass samples were first characterized by infrared spectroscopy ( $\sim 35 \mathrm{~min}$ acquisition time), and subsequently by contact angle measurements. After adlayer deposition, the gold disk and slab electrodes were used immediately only for electrochemical measurements.

Three types of reaction vessels were utilized, depending upon the type of diffusional mass transport desired and the size of the substrate. The common staining jar (described in the Mass Transfer Model section) was used for the gold on glass slides, and resulted in a 
linear mass transport of thiols to the substrate. The polycrystalline bulk gold slabs utilized a custom-built linear diffusion reaction vessel. The vessel was comprised of quartz glass slides fused in a rectangular geometry with dimensions $(2 \times 3 \times 7.5 \mathrm{~cm})$. The gold slab was placed into one end of the vessel and bathed in solution, resulting in thiol diffusion along the long dimension of the vessel. Polycrystalline gold disk electrodes were immersed in solutions in glass jars of various dimensions, the most common of which was a common scintillation vial. The electrode was suspended from the lid approximately in the middle of the vessel.

\section{Infrared Reflection-Absorption Spectroscopy}

Infrared spectra were acquired with a Nicolet 740 FT-IR spectrometer, equipped with a liquid nitrogen cooled mercury cadmium telluride detector, by using $p$-polarized light incident at $80^{\circ}$. The sample chamber was purged with nitrogen for $20 \mathrm{~min}$ prior to data collection. Spectra were obtained by referencing 1024 sample scans to 1024 background scans at $4 \mathrm{~cm}^{-1}$ resolution (zero filled to $2 \mathrm{~cm}^{-1}$ ) with Happ-Genzel apodization. Background spectra were obtained using a gold slide coated with per-deuterated octadecanethiolate.

\section{Contact Angle Measurements}

Advancing $\left(\theta_{\mathrm{a}}\right)$ contact angles were measured using a Rame-Hart Model 100-00 115 goniometer with deionized water as the probe liquid. $\mathrm{A} \sim 5 \mu \mathrm{L}$ drop of water was dispensed onto the substrate surface using a 10- $\mu \mathrm{L}$ Gilson Pipetman. For $\theta_{\mathrm{a}}$ measurements, the pipet tip was slowly pulled away from the drop while increasing its volume.

\section{Electrochemistry}

Electrochemical measurements were carried out using a $\mathrm{CH}$ Instruments model 660B potentiostat, in a three-electrode cell using a silver/silver chloride, saturated sodium chloride electrode $(\mathrm{Ag} / \mathrm{AgCl}$, sat'd $\mathrm{NaCl})$ as the reference and a flame-cleaned, coiled platinum wire 
auxiliary electrode. Electrolyte solutions were degassed with high-purity nitrogen for $\sim 10$ min, and a blanket of nitrogen was kept over the solution throughout the experiments.

Heterogeneous electron-transfer rate constants $\left(k_{\text {app }}^{0}\right)$ were assessed with cyclic voltammetry (CV) and electrochemical impedance spectroscopy (EIS). For CV, Nicholson's method $^{82}$ was used for quasireversible cases, and the Butler-Volmer relationship for irreversible cases:

$$
k_{\text {app }}^{0}=\frac{i^{0}}{n F A C_{b}}
$$

where $i^{0}$ is the faradaic current at the formal reduction potential of the redox probe, $C_{\mathrm{b}}$ is its bulk concentration, $n$ is the electron stoichiometry, $A$ is the electrode area, and $F$ is the Faraday. EIS measurements of the charge transfer resistance utilized an equivalent circuit consisting of a modified Randles circuit ${ }^{83}$ with a Warburg impedance in series with the charge transfer resistance, both in parallel with a double-layer capacitance (represented by a constant phase element with an exponent equal to 0.85 ). The constant phase element is used to account for heterogeneous features of the electrodes (i.e., adlayer defects, different crystal planes, and topographical features, where an exponent of unity represents a uniformly capacitive electrode). Rate parameters are determined by fitting the equivalent circuit using the CHI 5.07 software package.

The EIS data agrees well with the voltammetric data, noting that for illustrative purposes we focus on the cyclic voltammetry data. Moreover, since a standard modified Randles circuit with only one charge transfer resistance was used to determine $k_{\text {app }}^{0}$ from EIS experiments, the obtained error exceeds that from CV; this is most likely due to the fact that partially covered gold electrodes present multiple electron-transfer sites with a distribution of rates that cannot be accounted for by the standard circuit. 


\section{$\underline{\text { Results and Discussion }}$}

In order to evaluate the impact of the two extremes in impingement models, several experimental methods were employed to follow the adsorption of hexanethiol onto gold as a function of assembly conditions (i.e., thiol concentration, immersion time, and deposition geometry). Infrared reflection absorption spectroscopy (IRRAS) and wetting measurements were used to interrogate the structural evolution of the adlayer under conditions in which linear diffusion dominates the delivery of the reactants to the gold surface (i.e., gold films coated on glass slide and glass staining jars). Heterogeneous electron-transfer kinetics measurements were carried out for adlayers deposited on polycrystalline bulk gold electrodes in which the substrate-reaction vessel geometry and immersion times could be manipulated in order to characterize the effects of both radial and linear diffusional transport. Thus, by following the efficiency of the adlayers in serving as barriers to heterogeneous electron transfer from solution-based redox couples, differences in barrier properties were assessed for architectures formed by radial or linear diffusion.

\section{Polycrystalline Gold on Glass}

The sample size necessary for carrying out structural and wetting characterizations limit the choice of deposition geometry. For example, a deposition container that would allow the application of the radial diffusion model for a $2.5 \times 2.5 \mathrm{~cm}$ substrate, a size approaching the lower limit for IRRAS, must be at least $12.5 \times 12.5 \times 12.5 \mathrm{~cm}$ (container dimensions five times larger than the substrate dimensions; this constraint was chosen as a conservative value to ensure accurate model calculations). Moreover, to obtain a diffusion distance of $12.5 \mathrm{~cm}$, a minimum immersion time of $361 \mathrm{~h}$ (from Equation 5) is required. Thus, structural assessments of monolayers deposited in the radial diffusion regime using IRRAS are impractical. 
Therefore, assessment of interfacial properties by IRRAS and contact angle measurements necessitate the use of the linear impingement model. In addition, the use of staining jars for monolayer deposition dictate that the linear impingement model is applied as the $x$ and $y$ dimensions of the container match that of the substrate (i.e., the jar effectively confines the solution to a box-like volume element). Importantly, the evolution of adlayer properties is followed during the initial stages of growth and reorganization (i.e., beginning from partial monolayer impingement) where the most dramatic changes are expected. Moreover, the impingement rate, which can be controlled by varying the thiol concentration, during these early stages may dictate the way in which the adlayer structure develops throughout more advanced stages.

IRRAS

The average structure and orientation of alkanethiolates on gold can be assessed from the positions and intensities of bands in the $\mathrm{C}-\mathrm{H}$ stretching region. ${ }^{15,84}$ For example, the intensity of a vibrational mode at a highly reflective metal surface is maximized for a given surface concentration when the transition dipole is perpendicular to the surface; a more tilted orientation has a less intense absorption. ${ }^{15}$ Thus, by correlating the spatial orientations of several transition dipole moments, insights into the molecular structure of the adlayer and its evolution can be developed.

Figure 4 presents spectra for a series of hexanethiolate monolayers prepared by immersion in 1.0 and $10.0 \mu \mathrm{M}$ hexanethiol in ethanol. Mode assignments and orientations as well as peak positions are given in Table 2 along with the orientation of some transition dipoles. 


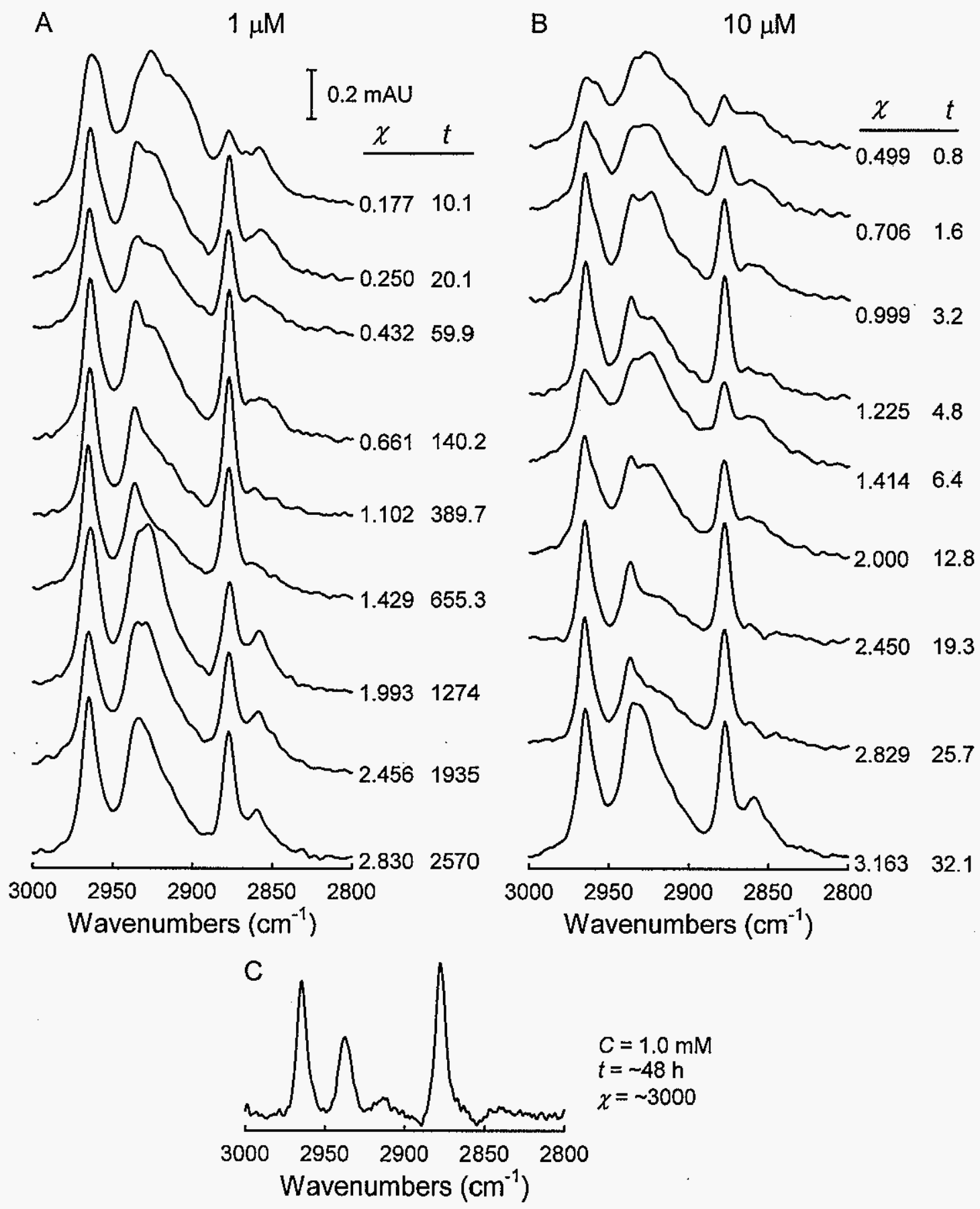

Figure 4. Infrared reflection absorption spectra for hexanethiolate on $\mathrm{Au}$ prepared by immersing a gold sample in A) $1.0 \mu \mathrm{M}, \mathrm{B}) 10.0 \mu \mathrm{M}$, and C) $1.0 \mathrm{mM}$ hexanethiol in ethanol. $\chi$ and $t(\mathrm{~min})$ are indicated at the right of each spectrum. 
Table 2. C-H stretching mode peak positions $\left(\mathrm{cm}^{-1}\right)$ for $\mathrm{CH}_{3}\left(\mathrm{CH}_{2}\right)_{5} \mathrm{SH}^{1}$

\begin{tabular}{|c|c|c|c|c|c|c|}
\hline $\mathrm{v}_{\mathrm{a}}\left(\mathrm{CH}_{3}\right)_{\mathrm{ip}}{ }^{a}$ & $\mathrm{v}_{\mathrm{a}}\left(\mathrm{CH}_{3}\right)_{\mathrm{op}}{ }^{b}$ & $\begin{array}{c}\mathrm{v}_{\mathrm{s}}\left(\mathrm{CH}_{3}\right)_{\mathrm{FR}}{ }^{\mathrm{c}} \\
\mathrm{r}_{\mathrm{FR}}{ }^{+}\end{array}$ & $\begin{array}{c}\mathrm{V}_{\mathrm{a}}\left(\mathrm{CH}_{2}\right)_{\mathrm{FR}}{ }^{d} \\
\mathrm{~d}_{\mathrm{FR}}^{-}\end{array}$ & $\begin{array}{c}\mathrm{v}_{\mathrm{a}}\left(\mathrm{CH}_{2}\right)^{e}{ }^{e} \\
\mathrm{v}_{\mathrm{s}}\left(\mathrm{CH}_{2}\right)_{\mathrm{FR}} \\
\mathrm{d}_{\mathrm{FR}}{ }^{+}, \mathrm{d}^{-}\end{array}$ & $\begin{array}{c}v_{\mathrm{s}}\left(\mathrm{CH}_{3}\right)^{c} \\
\mathrm{r}^{+}\end{array}$ & $\begin{array}{c}v_{s}\left(\mathrm{CH}_{2}\right)^{\gamma} \\
\mathrm{d}_{\beta}^{+} \\
\end{array}$ \\
\hline 2965 & 2956 & 2935 & 2925 & 2909 & 2878 & 2859 \\
\hline $\begin{array}{ll}\text { I } & \text { Assign } \\
& \text { Porter, } \\
& \text { Rowntr } \\
& \text { Girolan } \\
a & \perp \text { to the } \\
{ }_{b} & \perp \text { to the } \\
c & \| \text { to the } \\
d & \perp \text { to the } \\
e & \text { the assi } \\
f & \| \text { to the }\end{array}$ & $\begin{array}{l}\text { nts and orie } \\
\text { D. J. Am. } \\
\text { P. A. J. Ph } \\
\text { G. S.; Nuzz } \\
\mathrm{H}_{3}-\mathrm{CH}_{2} \text { bor } \\
-\mathrm{CH}_{3} \text { bond } \\
\mathrm{H}_{3}-\mathrm{CH}_{2} \text { bor } \\
\text {-C-C plane } \\
\text { ment of this } \\
\text {-C-C plane }\end{array}$ & $\begin{array}{l}\text { ions: all frot } \\
\text { m. Soc. } 199 \\
\text { Chem. } 1996 \\
\text { R. G. J. Phys } \\
\text { n the C-C-C } \\
\text { o the C-C-C }\end{array}$ & $\begin{array}{l}\text { alczak, M. N } \\
\text { 3, 2370-8 ex } \\
\text { 19917-26; } \\
\text { em. 1995, } 95 \\
\text { e }\end{array}$ & $\begin{array}{l}\text { hung, C.; S } \\
\mathrm{v}_{\mathrm{a}}\left(\mathrm{CH}_{2}\right) \text { ta } \\
\text { tetler, M. J. } \\
\text { 269-78. }\end{array}$ & $\begin{array}{l}\text { S. M.; } \\
\text { from Tr } \\
\text { lanner, W }\end{array}$ & $\begin{array}{l}\text { rig, C. A.; } \\
\text {, K. D.; }\end{array}$ \\
\hline
\end{tabular}

\section{General IRRAS Signatures}

An analysis of the spectral series shown in Figure 4 yields several important insights regarding the structural evolution of the adlayer as a function of immersion time and $\chi$. However, we note there are a few spectral features yet to be assigned and trends in some features that remain poorly understood. During the initial stages of formation, STM images often reveal stripped phases that correspond to all-trans chains oriented parallel to the gold surface. ${ }^{16}$ As assembly proceeds, the chains become directed in an increasingly perpendicular orientation until the limiting values for tilt and twist are reached (ca. $30^{\circ}$ and $55^{\circ}$ ). ${ }^{8,14,85-88}$ The changes in the IRRAS spectra in Figure 4 qualitatively follow this mechanistic description, demonstrating that the impingement model can yield insights into the structural changes that accompany nucleation and growth. A structural description of two example adlayers at short and long immersion times will serve to illustrate the analysis of the IRRAS data.

The transition dipole vector $\nu_{\mathrm{s}}\left(\mathrm{CH}_{2}\right)$ at $2859 \mathrm{~cm}^{-1}$ is aligned parallel to the $\mathrm{C}-\mathrm{C}-\mathrm{C}$ backbone and bisects the $\mathrm{H}-\mathrm{C}-\mathrm{H}$ bond. This mode is easily identified at low impingement numbers (e.g., the spectra in Figure 4A for $\chi=0.177$ ), indicating that a portion of the adsorbates have an alkyl chain that is oriented parallel to the surface normal (a "flat-lying" 
state). There are two different orientations for the flat-lying state, "edge-on" and "flat-on," depending upon whether the plane of the carbon backbone is perpendicular or parallel to the surface. Thus, the presence of additional bands (e.g., $v_{a}\left(\mathrm{CH}_{2}\right)$ ) with significant absorbance at low $\chi$ values indicates no discernable preference for the orientation of the flat-lying molecules.

The spectral signature at larger $\chi$-values is indicative of a more perpendicular orientation ("standing-up"). For example, the larger intensity for the $v_{\mathrm{s}}\left(\mathrm{CH}_{3}\right)$ band at 2878 $\mathrm{cm}^{-1}$ in the spectrum for larger $\chi$-values (e.g., Figure $4 \mathrm{~A}$ for $\chi=2.830$ ) signifies the chains are oriented more perpendicular to the surface; the transition dipole is oriented parallel to the $\mathrm{CH}_{3}-\mathrm{CH}_{2}$ bond.

Overall, the strengths of the bands in the IRRAS spectra point to changes in the orientation of the alkyl chains as $\chi$ increases. That is, the alkyl chains become increasingly aligned towards the surface normal as impingement increases. At the longest immersion times tested, a comparison of the spectrum for an adlayer prepared with much higher impingement $(\chi=\sim 3000)$ shows that the adlayer growth is still incomplete. In addition, although the data is not shown, similar trends were observed for experiments in which butanethiol was employed.

\section{Comparison of Impingement Rates}

The evolution of the IRRAS spectra with immersion time (or impingement) in Figure $4 \mathrm{~A}$ with respect to that in Figure $4 \mathrm{~B}$ reveals several differences. The rate of thiol impingement and the magnitude of $\chi$ can dictate the structure of the resulting adlayer, especially at low $\chi$. For example, the $\chi$-value of unity for $10.0 \mu \mathrm{M}$ solution requires an immersion time of $3.2 \mathrm{~min}$, whereas the $1.0 \mu \mathrm{M}$ solution requires $320 \mathrm{~min}$. The spectral signature for a $\chi=0.999$ in the $10.0 \mu \mathrm{M}$ series suggests the chains for this adlayer are less tilted than those for the layer prepared with the $1.0 \mu \mathrm{M}$ solution and a $\chi=1.102$. These 
spectra therefore demonstrate that impingement rate has a significant effect on the resulting adlayer structure, which is in all likelihood intimately tied to the time required for reorganization of the adlayer toward its most stable architecture.

Higher $\chi$ values as a result of the higher concentration also result in a more stable adlayer structure. This situation is exemplified by comparing the spectra for the adlayer where $\chi$ is 0.250 (20 min immersion) for the $1.0 \mu \mathrm{M}$ solution to that in which $\chi$ is 2.450 (19 min immersion) for the $10.0 \mu \mathrm{M}$ solution. Thus, these adlayers were prepared using similar immersion times, but at two different concentrations. Immersion in the $10.0 \mu \mathrm{M}$ solution results in a more fully covered substrate, as expected from the larger $\chi$-value. In addition, the spectra suggest the adlayer is more organized for the higher impingement value. However, note that both the 1.0 and $10.0 \mu \mathrm{M}$ deposition solutions result in adlayers that become structurally similar as $\chi$ becomes large.

\section{Contact Angle}

Wetting measurements provide a facile means for assessment of the surface free energy of a solid. ${ }^{89}$ Thus, by measuring $\theta_{\mathrm{a}}$ with water as the probe liquid on the same substrates used in IRRAS measurements, general correlations between the evolution of the surface free energy and adlayer architecture can be made. For reference, bare gold substrates are wetted by water, hydrophobic surfaces, such as paraffin and hexamethylethane, present methyl groups and yield $\theta_{\mathrm{a}}$ values of $\sim 130^{\circ},{ }^{12}$ and exposed methylene groups (e.g., polyethylene) exhibit $\theta_{\mathrm{a}} \sim 102^{\circ} .^{90}$ The most hydrophobic hexanethiolate monolayer which we have observed (prepared using a $>24 \mathrm{~h}$ immersion in $1.0 \mathrm{mM}$ hexanethiol) has a $\theta_{\mathrm{a}}$ value of $114^{\circ}$. The depth sensitivity of the contact angle measurement must also be considered. ${ }^{91}$ If the average thickness of a dielectric coating is less than the depth sensitivity, the droplet may also respond to the presence of the substrate. 
The expected evolution of $\theta_{a}$ with immersion time is derived from several possible contributions. First, as the thiolate adlayer increases in packing density, the surface presents an increased number of methyl groups per unit area, with a corresponding decrease in exposed methylene groups. Second, as the coverage increases, the direct contact of water with gold will decrease. Third, as the thickness increases, the gold surface becomes more spatially separated from the contacting liquid. Each of these factors will cause an increase in the contact angle, eventually reaching an expected limiting value of $114^{\circ}$.

Figure 5 shows contact angles for water $\left(\theta_{\mathrm{a}}\right)$ as a function of $\chi$ for both the 1.0 and $10.0 \mu \mathrm{M}$ solution concentrations. The error bars included in Figure 5 are two standard deviations, five measurements on one substrate, in width. A further discussion of the sample-to-sample reproducibility is also important. We envision, during the initial placement of the substrate into the thiol solution, a finite amount of convective mass transport occurs. Samples at short immersion time would therefore suffer from a larger percentage of nondiffusional thiol transport. In contrast, for long immersion times, the contributions from convection are overwhelmed by diffusional mass transport. Therefore, we are uncertain of the sample-to-sample reproducibility at short times, which is more important in Figure 5B due to the much shorter immersion times required to match the impingements of Figure 5A (note the unit of the time axes on the two plots). As an example, we reproduced sample at $t=6.4$ and $12.8 \mathrm{~min}$ for the $10.0 \mu \mathrm{M}$ trial. As shown, the difference in contact angle between the two samples was higher for the shorter time. We expect this variability to increase continually at lower immersion times.

Overall, the contact angles approach higher values as $\chi$ increases. Data for the adlayers formed in the $10.0 \mu \mathrm{M}$ solution did not move towards as large of contact angles as those with the $1.0 \mu \mathrm{M}$ solution. This observation implies less structural order (i.e, packing density or orientation) for the adlayers prepared using the higher concentration, even when the overall impingement is identical, and suggests that in this case insufficient time is 
allowed for adlayer reorganization. That is, for an identical $\chi$-value, a higher solution concentration only allows a shorter immersion time and therefore a shorter period for reorganization.
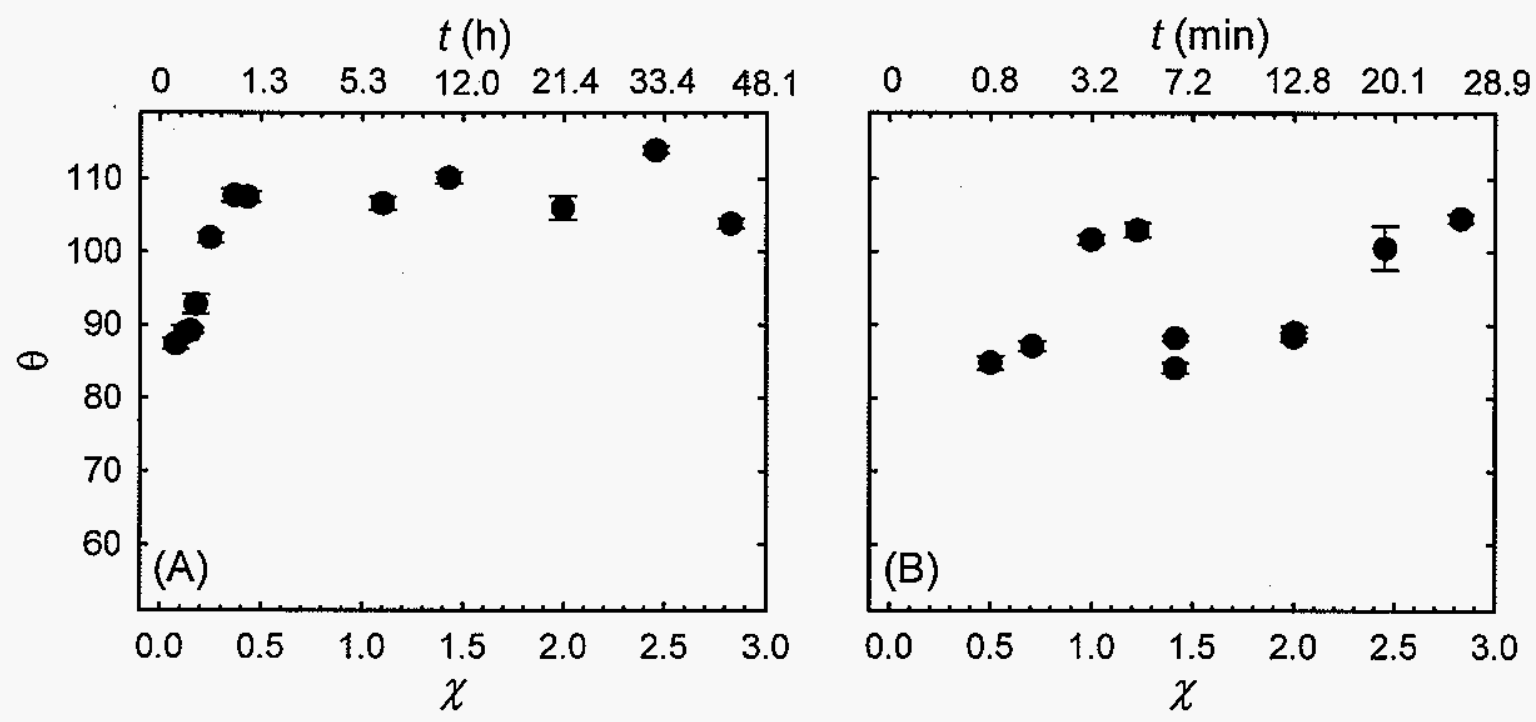

Figure 5. Advancing $\left(\theta_{a}\right)$ contact angles for water on hexanethiolate monolayers prepared by immersion of gold-coated glass substrates in $\mathrm{A}$ ) $1.0 \mu \mathrm{M}$ and B) $10.0 \mu \mathrm{M}$ hexanethiol. Each data point is a combination of five measurements on the same substrate, with error bars corresponding to two standard deviations.

A dramatic progression of the contact angle values at $\chi<0.5$ for the $1.0 \mu \mathrm{M}$ solution, suggests that the adlayer assembly process is progressing. The contact angle values approach $114^{\circ}$, which like the conclusions drawn from the IRRAS data, indicates that the chains are aligning more toward the surface normal. The $10.0 \mu \mathrm{M}$ trial does not reach as high a contact angle value, which implicates that sufficient time has be allowed for reorganization. In other words, for identical $\chi$-values, the longer immersion time (and correspondingly lower concentration) allows sufficient time for adlayer reorganization. At similar immersion times, a higher $\chi$-value results in an increased surface concentration, but not as well-packed structure as if time had been allowed for reorganization. As such, the contact angle data reinforce conclusions drawn from the IRRAS data. 


\section{Polycrystalline Bulk Gold}

The size of the gold disk electrodes utilized $\left(r_{\mathrm{e}}=0.080 \mathrm{~mm}\right)$ precludes IRRAS and contact angle characterizations, but were highly amendable to electrochemical measurements. Therefore, deposition geometry and solution conditions were selected to fit within the framework dictated by the radial model. In an attempt to establish an assessment of the effects of adlayer impingement of the linear vs. radial depositional model, polycrystalline bulk gold slabs were deposited linearly.

Measurements of heterogeneous electron-transfer rates are among the most sensitive (if not the most) metrics for interrogating adlayer quality. ${ }^{5}$ As such, the solution-based redox couple $\mathrm{Fe}(\mathrm{CN})_{6}^{4-/ 3-}$ was used as a probe, and electron-transfer rates for adlayers prepared under different conditions were measured. The two models were used to delineate conditions in which comparisons between the two cases would potentially be informative.

The rate constant $\left(k_{\text {app }}^{0}\right)$ of a heterogeneous electron-transfer reaction is known to undergo an exponential decrease as the thickness of a dielectric barrier increases. ${ }^{83}$ Thus, as the coverage and thickness of the hexanethiolate adlayer increases, a decrease in $k_{\text {app }}^{0}$ should be observed. For example, at uncoated gold, the value of $k_{\text {app }}^{0}$ for the $\mathrm{Fe}(\mathrm{CN})_{6}{ }^{4-/ 3-}$ couple is $10^{-2} \mathrm{~cm} \mathrm{~s}^{-1}$, which is the largest expected value. On the other hand, at an electrode coated with a fully extended, closest packed hexanethiolate layer, the rate is theoretically expected to be $\sim 10^{-6} \mathrm{~cm} \mathrm{~s}^{-1}$. We have occasionally observed rates of this magnitude for hexanethiolate monolayers on gold.

Figure 6 presents a series of cyclic voltammograms, while Table 3 compiles the extracted rates from both CV and EIS experiments. A careful analysis of the data by comparison of conditions, in which only a single variable was altered, enables several significant insights into adlayer growth. In addition, since both linear and radial geometries were tested, a direct comparison of the effect of electrode/vessel configuration can be made. 
Table 3. Electron-transfer rates for the solution-based redox couple $\mathrm{Fe}(\mathrm{CN})_{6}{ }^{4-}$.

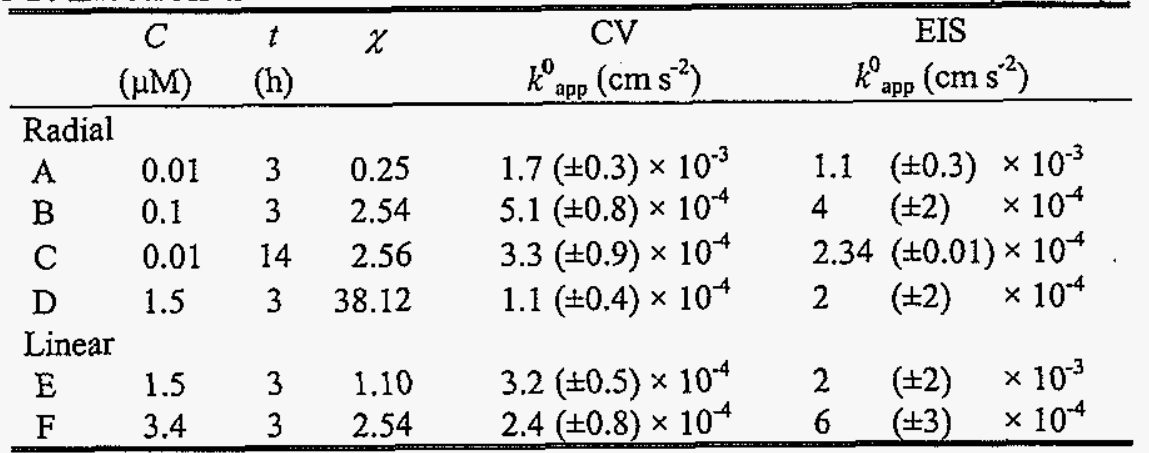

Data collected on 3 separate electrodes per trial (except $E$ with 2 electrodes), error consists of \pm one standard deviation.

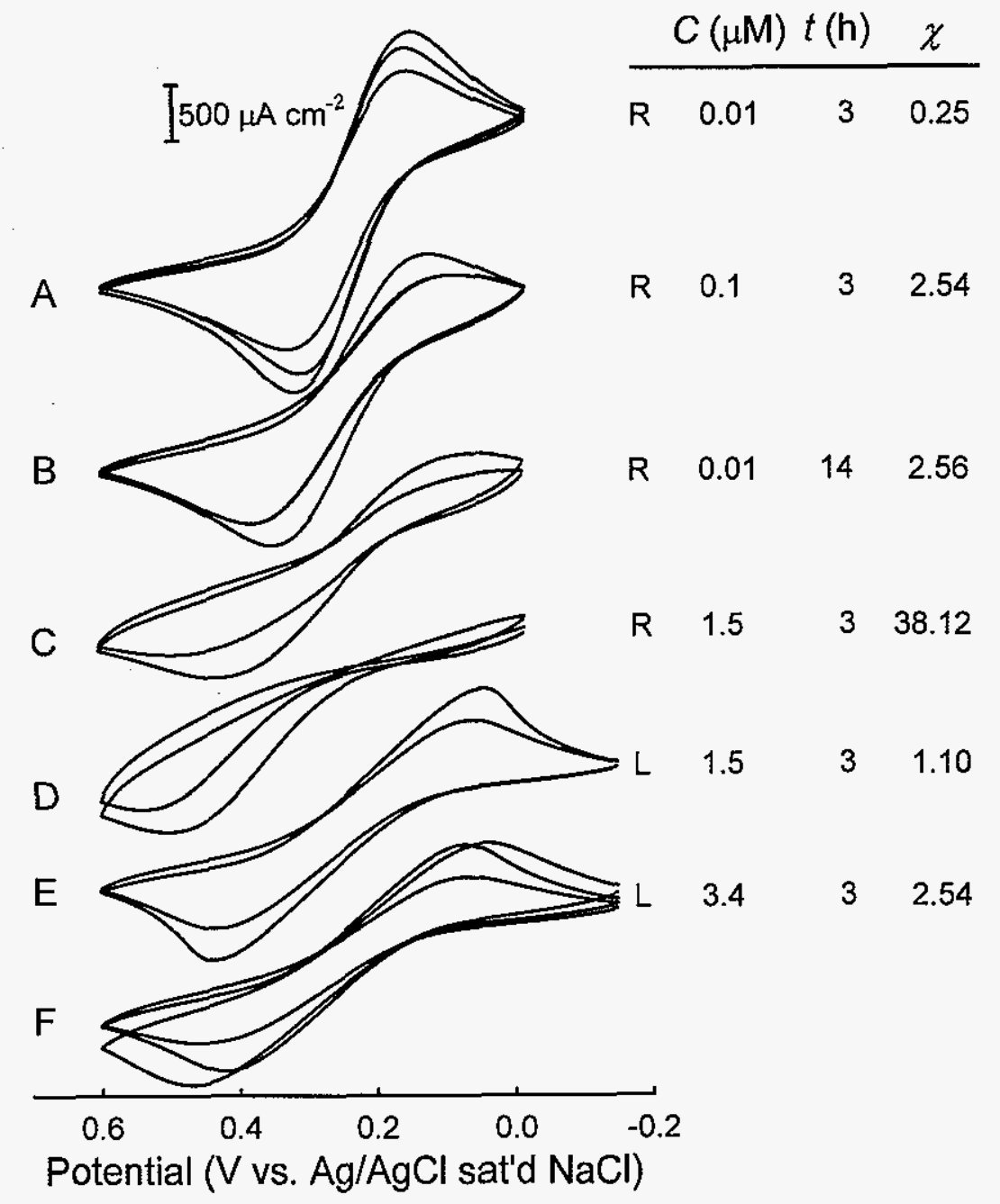

Figure 6. Cyclic voltammograms of hexanethiolate modified gold electrodes in $10 \mathrm{mM} \mathrm{Fe}(\mathrm{CN})_{6}{ }^{4-}$ in $0.125 \mathrm{M} \mathrm{NaF}$, and scan rate $100 \mathrm{mV} \mathrm{s}{ }^{-1}$. Deposition geometry $(\mathrm{R}=$ radial, $\mathrm{L}=$ linear), $\chi$, immersion time $(\mathrm{h})$, and solution concentration $(\mu \mathrm{M})$ are indicated at the right of each voltammogram. 


\section{Radial Diffusion Model}

First, we note that the electron-transfer rate for condition $\mathrm{A}$ is ten times less than that at an unmodified gold electrode, indicating the start of adlayer formation, as reflected by $\chi=0.25$. The rate for electrodes modified by immersion for similar times in thiol solutions of different concentrations, evident by comparison of Figures $6 \mathrm{~A}$ and $6 \mathrm{~B}$, show that there is a dramatic dependence of the efficiency of the hexanethiolate adlayer to serve as a barrier to electron transfer on $\chi$. Thus, when $\chi$ is 2.54 (Figure $6 \mathrm{~B}$ ), $k_{\text {app }}^{0}$ is $30 \%$ less than when $\chi$ is 0.25 (Figure 6A). This difference is the result of the 10-fold increase in the impingement number for the adlayer in the former case with respect to the latter. This observation suggests a more densely packed and thicker coating when $\chi$ is 2.54 , and is supported by the IRRAS data. However we add that these monolayers are not defect-free, as that the expected value of $k_{\text {app }}^{0}$ at an ideal barrier $\left(\beta=1.0 \AA^{-1}\right)$ is $\sim 10^{-6} \mathrm{~cm} \mathrm{~s}^{-1}$.

In contrast, comparison of the voltammograms in Figures $6 \mathrm{~B}$ and $6 \mathrm{C}$, created by electrodes with identical $\chi$-values but different thiol concentrations, illustrates the importance of impingement rate. That is, the adlayer with a longer immersion time is more effective at blocking heterogeneous electrolysis even though $\chi$ is nearly identical in both cases. This result suggests a more densely packed monolayer can form due to a lower level of nucleation and a longer time for reorganization.

Finally, Figure 6D completes the comparison of radial diffusion experiments by showing the effect of a much higher thiol concentration. In this case, $\chi$ is $\sim 38$, and the rate of electrolysis is the slowest of the four different test cases. However, we note that in other experiments we have observed rates of $\sim 10^{-6} \mathrm{~cm} \mathrm{~s}^{-1}$ for much larger values of $\chi$.

\section{Linear Diffusion Model}

The bulk gold slab electrodes were used in a custom-built linear diffusion reaction vessel as detailed in the Experimental section. The solution conditions were chosen for 
comparison with the experimental results of the radial diffusion model. For instance, the comparison of Figures $6 \mathrm{D}$ and $6 \mathrm{E}$ yields striking conclusions. In this case, two electrodes with the same solution conditions, but different deposition geometry exhibit significant differences: there is a decrease in observed rate for the radial case. Moreover, notice the marked differences in the overall impingement at the two substrates. From these data, one can infer that the increase in $\chi$ drives the formation of a more densely packed adlayer that then acts as a stronger barrier to the electrolysis of $\mathrm{Fe}(\mathrm{CN})_{6}{ }^{4-/ 3}$.

In addition, comparison of Figure $6 \mathrm{~B}$ and $6 \mathrm{~F}$ shows the case in which the overall impingement and immersion time is constant. In this example, the electron transfer rate is smaller for the linear case. This observation, again points to the importance of the initial impingement rate. The solution concentration in trial $\mathrm{F}$ is much larger than that in trial $\mathrm{B}$ due to the higher solution concentration. In this case, a more closely-packed adlayer results in $\mathrm{F}$ because a higher percentage of the adlayer forms initially and more time is allowed for the thiolates to reorganize; however, for high initial impingement rates and short immersion times this statement may not be valid. The key is that both the impingement rate and immersion time must be considered.

The culmination of these two comparisons shows that the deposition of adlayer components by either radial or linear diffusion does not result in the same adlayer structure or properties. In this way, for reproducible adlayer assembly, the deposition conditions (immersion time, solution concentration, and deposition geometry) must be carefully controlled.

\section{Conclusion}

This work has shown that immersion time, solution concentration, and deposition geometry are important aspects of adlayer preparation that must be carefully controlled to create modified surfaces exhibiting a reproducible structure and function. A mass transport 
model was developed to relate the impingement number to time, concentration, and deposition geometry. Equations were derived to relate these parameters within different deposition conditions, with both linear and radial diffusion considered. Experimentally, different substrate/vessel geometries were used to prepare adlayers with varying deposition conditions upon which the models were based. These films were characterized by IRRAS, wettability, and electrochemistry to verify model concepts.

The data included herein point toward three important conclusions: impingement number and rate, as controlled by immersion time and thiol concentration as well as deposition geometry are all extremely important variables in self-assembly. The difficulties discussed by $\mathrm{Ma}$ and Lennox ${ }^{40}$ in obtaining reproducible SAM structure and property for adlayers formed by ostensibly identical preparation procedures can be explained by these works. Therefore, future work involving the use of SAM films must carefully consider deposition geometry, solution concentration, and immersion time. In addition, this model will serve to facilitate discussion of adlayer deposition kinetics as they pertain to impingement of molecules to the surface.

\section{$\underline{\text { Acknowledgments }}$}

This work was supported, in part, by Eastman Chemical and the Ames Laboratory-US DOE. G.A.E. gratefully acknowledges a Conoco-Phillips graduate research fellowship. The Ames Laboratory is operated by Iowa State University for the U.S. Department of Energy under Contract W-7405-ENG-82.

\section{$\underline{\text { References }}$}

(1) Murray, R. W. "Chemically Modified Electrodes" In Electroanalytical Chemistry; Bard, A. J., Ed.; Marcel Dekker, Inc.: New York, 1984; Vol. 13, p 191-368.

(2) Molecular Design of Electrodes Surfaces; Murray, R. W., Ed.; John Wiley \& Sons, Inc.: New York, New York, 1992; Vol. XXII.

(3) Zhong, C.-J.; Porter, M. D. Anal. Chem. 1995, 67, 709A-15A. 
(4) Ulman, A. An Introduction to Ultrathin Organic Films From Langmuir-Blodgett to Self-Assembly; Academic Press: San Diego, CA, 1991.

(5) Finklea, H. O. "Electroanalytical Methods: Self-Assembled Monolayers on Electrodes" In Encylopedia of Analytical Chemistry; Meyers, R. A., Ed.; John Wiley \& Sons, Ltd: Chichester, 2000; Vol. 11, p 10090-115.

(6) Ulman, A. Chem. Rev. 1996, 96, 1533-54.

(7) Love, J. C.; Estroff, L. A.; Kriebel, J. K.; Nuzzo, R. G.; Whitesides, G. M. Chem. Rev. 2005, 105, 1103-69.

(8) Porter, M. D.; Bright, T. B.; Allara, D. L.; Chidsey, C. E. D. J. Am. Chem. Soc. 1987, $109,3559-68$.

(9) Widrig, C. A.; Chung, C.; Porter, M. D. J. Electroanal. Chem. 1991, 310, 335-59.

(10) Walczak, M. M.; Chung, C.; Stole, S. M.; Widrig, C. A.; Porter, M. D. J. Am. Chem. Soc. 1991, 113, 2370-8.

(11) Zhong, C.-J.; Brush, R. C.; Andregg, J.; Porter, M. D. Langmuir 1999, 15, 518-25.

(12) Bain, C. D.; Troughton, E. B.; Tao, Y.-T.; Evall, J.; Whitesides, G. M.; Nuzzo, R. G. J. Am. Chem. Soc. 1989, 111, 321-35.

(13) Laibinis, P. E.; Fox, M. A.; Folkers, J. P.; Whitesides, G. M. Langmuir 1991, 7, $3167-$ 73.

(14) Laibinis, P. E.; Whitesides, G. M.; Allara, D. L.; Tao, Y.-T.; Parikh, A. N.; Nuzzo, R. G. J. Am. Chem. Soc. 1991, 113, 7152-67.

(15) Allara, D. L.; Nuzzo, R. G. Langmuir 1985, 1, 52-66.

(16) Poirier, G. E.; Pylant, E. D. Science 1996, 272, 1145-8.

(17) McCarley, R. L.; Dunaway, D. J.; Willicut, R. J. Langmuir 1993, 9, 2775-7.

(18) Xu, S.; Cruchon-Dupeyrat, S. J. N.; Garno, J. C.; Liu, G.-Y.; Jennings, G. K.; Yong, T.-H.; Laibinis, P. E. J. Chem. Phys. 1998, 108, 5002-12.

(19) Aoki, H.; Umezawa, Y. Analyst 2003, 128, 681-5.

(20) Mandler, D.; Turyan, I. Electroanalysis 1996, 8, 207-13.

(21) Turyan, I.; Mandler, D. Anal. Chem. 1997, 69, 894-7.

(22) Turyan, I.; Mandler, D. Anal. Chem. 1994, 66, 58-63.

(23) Radford, P. T.; French, M.; Creager, S. E. Anal. Chem. 1999, 71, 5101-8.

(24) Forouzan, F.; Bard, A. J.; Mirkin, M. V. Isr. J. Chem. 1997, 37, 155-63. 
(25) Smalley, J. F.; Feldberg, S. W.; Chidsey, C. E. D.; Linford, M. R.; Newton, M. D.; Liu, Y.-P. J. Phys. Chem. 1995, 99, 13141-9.

(26) Sumner, J. J.; Weber, K. S.; Hockett, L. A.; Creager, S. E. J. Phys. Chem. B 2000, $104,7449-54$.

(27) Liu, B.; Bard, A. J.; Mirkin, M. V.; Creager, S. E. J. Am. Chem. Soc. 2004, 126, 148592.

(28) Creager, S. E.; Radford, P. T. J. Electroanal. Chem. 2001, 500, 21-9.

(29) Protsailo, L. V.; Fawcett, W. R. Electrochim. Acta 2000, 45, 3497-505.

(30) Brevnov, D. A.; Finklea, H. O.; Ryswyk, H. V. J. Electroanal. Chem. 2001, 500, 1007.

(31) Becka, A. M.; Miller, C. J. J. Phys. Chem. 1992, 96, 2657-68.

(32) Becka, A. M.; Miller, C. J. J. Phys. Chem. 1993, 97, 6233-9.

(33) Miller, C.; Cuendet, P.; Grätzel, M. J. Phys. Chem. 1991, 95, 877-86.

(34) Miller, C.; Grätzel, M. J. Phys. Chem. 1991, 95, 5225-33.

(35) Truong, K. D.; Rowntree, P. A. Prog. Surf. Sci. 1995, 50, 207-16.

(36) Truong, K. D.; Rowntree, P. A. J. Phys. Chem. 1996, 100, 19917-26.

(37) Walczak, M. M.; Alves, C. A.; Lamp, B. D.; Porter, M. D. J. Electroanal. Chem. 1995, 396, 103-14.

(38) Zhong, C.-J.; Zak, J.; Porter, M. D. J. Electroanal. Chem. 1997, 421, 9-13.

(39) Creager, S. E.; Hockett, L. A.; Rowe, G. K. Langmuir 1992, 8, 854-61.

(40) Ma, F.; Lennox, R. B. Langmuir 2000, 16, 6188-90.

(41) Camillone, N., III Langmuir 2004, 20, 1199-206.

(42) Hahner, G.; Wöll, C.; Buck, M.; Grunze, M. Langmuir 1993, 9, 1955-8.

(43) Qu, D.; Morin, M. J. Electroanal. Chem. 2002, 524-525, 77-80.

(44) Subramanian, R.; Lakshminarayanan, V. Electrochim. Acta 2000, 45, 4501-9.

(45) Cohen-Atiya, M.; Mandler, D. J. Electroanal. Chem. 2003, 550-551, 267-76.

(46) Pan, W.; Durning, C. J.; Turro, N. J. Langmuir 1996, 12, 4469-73.

(47) Karpovich, D. S.; Blanchard, G. J. Langmuir 1994, 10, 3315-22.

(48) Bain, C. D.; Whitesides, G. M. J. Am. Chem. Soc. 1989, 111, 7164-75. 
(49) Schreiber, F. J. Phys.: Condens. Matter 2004, 16, R881-R900.

(50) Sandhyarani, N.; Pradeep, T. Int. Rev. Phys. Chem. 2003, 22, 221-62.

(51) Schwartz, D. K. Annu. Rev. Phys. Chem. 2001, 52, 107-37.

(52) Peterlinz, K. A.; Georgiadis, R. Langmuir 1996, 12, 4731-40.

(53) Schessler, H. M.; Karpovich, D. S.; Blanchard, G. J. J. Am. Chem. Soc. 1996, 118, 9645-51.

(54) Biebuyck, H. A.; Bain, C. D.; Whitesides, G. M. Langmuir 1994, 10, 1825-31.

(55) Jung, L. S.; Campbell, C. T. J. Phys. Chem. B 2000, 104, 11168-78.

(56) Jung, L. S.; Campbell, C. T. Phys. Rev. Lett. 2000, 84, 5164-7.

(57) Nuzzo, R. G.; Zegarski, B. R.; Dubois, L. H. J. Am. Chem. Soc. 1987, 109, 733-40.

(58) Buck, M.;.Grunze, M.; Eisert, F.; Fischer, J.; Träger, F. J. Vac. Sci. Technol. A 1992, 10, 926-9.

(59) Xu, S.; Laibinis, P. E.; Liu, G.-Y.J. Am. Chem. Soc. 1998, 120, 9356-61.

(60) Eberhardt, A.; Fenter, P.; Eisenberger, P. Surf. Sci. 1998, 397, L285-L90.

(61) Hu, K.; Bard, A. J. Langmuir 1998, 14, 4790-4.

(62) Godin, M.; Williams, P. J.; Tabard-Cossa, V.; Laroche, O.; Beaulieu, L. Y.; Lennox, R. B.; Grütter, P. Langmuir 2004, 20, 7090-6.

(63) Jung, Ch.; Dannenberger, O.; Xu, Y.; Buck, M.; Grunze, M. Langmuir 1998, 14, $1103-7$.

(64) Bensebaa, F.; Voicu, R.; Huron, L.; Ellis, T. H.; Kruus, E. Langmuir 1997, 13, 533540.

(65) Yamada, R.; Uosaki, K. Langmuir 1997, 13, 5218-21.

(66) Kim, H. J.; Kwak, S.; Kim, Y. S.; Seo, B. I.; Kim, E. R.; Lee, H. Thin Sold Films 1998, 327-329, 191-4.

(67) Yamada, R.; Uosaki, K. Langmuir 1998, 14, 855-61.

(68) Schreiber, F.; Eberhardt, A.; Leung, T. Y. B.; Schwartz, P.; Wetterer, S. M.; Lavrich, D. J.; Berman, L.; Fenter, P.; Eisenberger, P.; Scoles, G. Phys. Rev. B 1998, 57, 12476-81.

(69) Lavrich, D. J.; Wetterer, S. M.; Bernasek, S. L.; Scoles, G. J. Phys. Chem. B 1998, $102,3456-65$. 
(70) Jung, L. S.; Campbell, C. T.; Chinowsky, T., M.; Mar, M. N.; Yee, S. S. Langmuir 1998, 14, 5636-48.

(71) Schwartz, P.; Schreiber, F.; Eisenberger, P.; Scoles, G. Surf. Sci. 1999, 423, 208-24.

(72) Himmelhaus, M.; Eisert, F.; Buck, M.; Grunze, M. J. Phys. Chem. B 2000, 104, 57684.

(73) Yang, C. S.-C.; Richter, L. J.; Stephenson, J. C.; Briggman, K. A. Langmuir 2002, 18, 7549-56.

(74) Dannenberger, O.; Buck, M.; Grunze, M. J. Phys. Chem. B 1999, 103, 2202-13.

(75) Kawasaki, M.; Sato, T.; Tanaka, T.; Takao, K. Langmuir 2000, 16, 1719-28.

(76) Sobocinski, R. L.; Pemberton, J. E. Langmuir 1992, 8, 2049-63.

(77) Jones, V. W.; Kenseth, J. R.; Porter, M. D.; Mosher, C. L.; Henderson, E. Anal. Chem. 1998, 70, 1233-41.

(78) Zhong, C.-J.; Woods, N. T.; Dawson, G. B.; Porter, M. D. Electrochem. Commun. 1999, $1,17-21$.

(79) Chandrasekhar, S. Rev. Mod. Phys. 1943, 15, 1-89.

(80) Green, J.-B. D. Scanning Probe Microscopies for the Characterization of Interfaces: Studies of Alkyl Thiolate Monolayers at Gold, Ph.D. Dissertation, Iowa State University, Ames, IA, 1996.

(81) Dickertmann, D.; Schultze, J. W.; Vetter, K. J. J. Electroanal. Chem. 1974, 55, 42943.

(82) Nicholson, R. S. Anal. Chem. 1965, 37, 1351-5.

(83) Bard, A. J.; Faulkner, L. R. Electrochemical Methods: Fundamentals and Applications; 2nd ed.; John Wiley \& Sons, Inc.: New York, 2001.

(84) Snyder, R. G.; Strauss, H. L.; Elliger, C. A. J. Phys. Chem. 1982, 86, 5145-50.

(85) Parikh, A. N.; Allara, D. L. J. Chem. Phys. 1992, 96, 927-45.

(86) Nuzzo, R. G.; Korenic, E. M.; Dubois, L. H. J. Chem. Phys. 1990, 93, 767-73.

(87) Nuzzo, R. G.; Fusco, F. A.; Allara, D. L. J. Am. Chem. Soc. 1987, 109, 2358-68.

(88) Dubois, L. H.; Nuzzo, R. G. Annu. Rev. Phys. Chem. 1992, 43, 437-63.

(89) Kolasinski, K. Surface Science: Foundations of Catalysis and Nanoscience; John Wiley \& Sons, Ltd: New York, 2001.

(90) Holmes-Farley, S. R.; Reamey, R. H.; McCarthy, T. J.; Deutch, J.; Whitesides, G. M. Langmuir 1985, 1, 725-40. 
(91) Bain, C. D.; Whitesides, G. M. J. Am. Chem. Soc. 1988, 110, 5897-8. 


\title{
CHAPTER 5: HIGH RESOLUTION MAPPING OF COMPOSITIONAL DIFFERENCES AT ELECTRODE INTERFACES BY ELECTRIC FORCE MICROSCOPY
}

\author{
G. A. Edwards, J. D. Driskell, A. J. Bergren, R. J. Lipert, and M. D. Porter \\ Ames Laboratory-U.S.D.O.E., Department of Chemistry, and the Institute for Combinatorial \\ Chemistry, Iowa State University, Ames, Iowa 50011 \\ A manuscript published in Technical Proceedings of the 2005 Nanotechnology Conference \\ and Trade Show, 2005, 2, 722-725.*
}

\begin{abstract}
$\underline{\text { Abstract }}$
This manuscript examines the mechanistic basis for the ability of electric force microscopy (EFM) to map terminal group differences of spatially patterned organic monolayers. It compares the experimentally observed EFM response to that from modeling calculations of the dipole moment of gold-bound adlayers prepared from a series of benzyl mercaptans, and serves as a starting point for gaining insight into the contrast mechanism. While preliminary, the results show that the dipole moment of the adlayer plays an important role in the contrast mechanism.
\end{abstract}

* ๑ 2005 NSTI http://nsti.org. Reprinted and revised, with permission, from the Technical Proceedings of the 2005 NSTI Nanotechnology Conference and Trade Show, Volume 2, pp. 722-725, May 8-12, 2005, Anaheim, CA, U.S.A. 


\section{$\underline{\text { Introduction }}$}

Scanning probe microscopic characterizations of organic thin films are widely utilized to investigate a range of interfacial processes (e.g., electrocatalysis, corrosion inhibition, conductivity of organic electronic devices, and biocompatibility). ${ }^{1}$ Recent reports have described the ability of electric force microscopy (EFM), ${ }^{2}$ an offshoot of atomic force microscopy, to map the terminal group differences of patterned organic monolayers that are buried under a thick $(\sim 500 \mathrm{~nm})$ film of an organic polymer. ${ }^{3}$ This presentation describes preliminary findings from experiments that seek to unravel the mechanistic basis of the contrast mechanism.

There are two steps in an EFM experiment. The first is a line-scan characterization of sample topography, which is usually collected in tapping mode. The electronic properties of the sample are interrogated on a second pass across the sample in lift-mode (i.e., the tip is rastered across the same line at a constant tip-sample separation as determined by the previous topographic line-scan). In the second pass, a dc bias voltage is applied across a gold-coated tip and a grounded conductive substrate. The electrical forces $\left(F_{\text {elec}}\right)$ interacting with the oscillating tip can be qualitatively represented by Equation $1,{ }^{4}$ where $C$ is the capacitance of the media between the tip and sample, $V_{\text {sample }}$ and $V_{\text {tip }}$ are the voltages of the sample and tip, respectively, and $z$ is the tip-sample separation. This formulation neglects contributions from the pyramidal-shape of the tip as well as the extended structure of the cantilever by treating the system as a parallel plate capacitor.

$$
F_{\text {clec }}=\frac{\partial C}{\partial z} \frac{\left(V_{\text {tip }}-V_{\text {sample }}\right)^{2}}{2}
$$

Force measurements can be accomplished by monitoring the amplitude or phase shift of the tip oscillation, with phase shift being the more sensitive of the two approaches. The phase shift $(\Delta \Phi)$ is proportional to the force gradient between the tip and sample, as shown in Equation 2. This equation indicates that a plot of $\Delta \Phi$ with respect to $V_{\text {tip }}$ will have a parabolic 
shape with respect to a change in the tip-sample voltage. The parabolic width (e.g., the length of the latus rectum, $L R$ ) is dependant on the capacitance of the media between the tip and sample and the minimum (i.e., the parabolic vertex), where $V_{\text {tip }}$ and $V_{\text {sample }}$ are identical. Therefore, the adlayer identity controls the capacitance and voltage gradient between the tip and substrate, which gives rise to the image contrast mechanism.

$$
\Delta \Phi \propto \frac{\partial F_{\text {elec }}}{\partial z} \propto \frac{\partial^{2} C}{\partial z^{2}} \frac{\left(V_{\text {tip }}-V_{\text {sample }}\right)^{2}}{2}
$$

This paper utilizes a set of gold substrates, which are modified with five different para-substituted benzyl thiolates as a test system to begin to elucidate the interfacial properties that contribute to image contrast. Molecular modeling is utilized to predict how these monomolecular films will modify the electronic properties of the gold-air interface, such that the expected surface potential $\left(\Delta U_{\text {model }}\right)$ can be calculated. Electrochemical measurements are presented to confirm adlayer deposition, determine an experimental double layer capacitance $\left(C_{\mathrm{dl}}\right)$, and estimate adlayer surface coverage $(\Gamma)$. This system of films is experimentally interrogated utilizing EFM to measure the interfacial electronic properties. The model developed to predict EFM contrast is then compared to the experimentally observed values.

\section{Experimental}

\section{Chemicals}

Absolute ethanol was purchased from Aaper Alcohol. The para-substituted benzyl mercaptans $(\mathrm{H}, t$-butyl, $\mathrm{Cl}, \mathrm{F}$, and $\mathrm{Br})$ and $\mathrm{NaOH}$ (semiconductor grade) were obtained from Aldrich. All chemicals were used as received. 


\section{Substrate and Monolayer Preparation}

Template-stripped gold (TSG) was prepared as previously reported. ${ }^{5,6}$ In brief, $300 \mathrm{~nm}$ of gold was resistively evaporated at a pressure of $7.5 \times 10^{-7}$ Torr onto silicon(111) wafers (University Wafer) at $0.2 \mathrm{~nm} \mathrm{~s}^{-1}$. Microscope slides (Fisher, $1 \times 1 \mathrm{~cm}$ ) were epoxied to the gold surface using Epo-Tek 377 (Epoxy Technology), and cured at $150^{\circ} \mathrm{C}$ for $105 \mathrm{~min}$. Samples were removed from the silicon wafer, and immediately immersed for $18 \mathrm{~h}$ in $1.0 \mathrm{mM}$ ethanolic thiol solutions, removed from solution, rinsed copiously with ethanol, and dried. The resulting gold surface has an electrochemical roughness factor of $1.3 .^{6}$

\section{Molecular Modeling}

Molecular models of the aromatic thiols were created by energy minimization using Chem3D Ultra 8.0. ${ }^{7}$ The dipole moments were calculated utilizing the CS MOPAC Pro package. The MOPAC software employs the Parameterized Model (revision 3, PM3) to generate the potential energy function with a closed shell wave function. Dipole moments were calculated using the Mulliken charge approximation. The three-dimensional molecular coordinates, in conjunction with the dipole vectors, were then utilized to determine the thickness and magnitude of the dipole moment (parallel to the alignment of the S-H bond) of the adlayers.

\section{Electrochemistry}

Electrochemical measurements were carried out using a CH Instruments model 600A potentiostat and a three-electrode cell with a platinum wire auxiliary electrode and a silver/silver chloride (saturated sodium chloride) reference electrode. All potentials are reported with respect to this reference. A solution of $0.5 \mathrm{M} \mathrm{NaOH}$ in high purity water was utilized for all experiments. Solutions were purged with nitrogen for $10 \mathrm{~min}$ prior to electrochemical measurements, and a blanket of nitrogen was held over the solution throughout each experiment. 
The interfacial capacitance $\left(C_{\mathrm{dl}}\right)$ of the samples were determined utilizing cyclic voltammetry (CV). ${ }^{8} \mathrm{CVs}$ were collected at three sweep rates $\left(50,75\right.$, and $\left.100 \mathrm{mV} \mathrm{s}^{-1}\right)$ by scanning between $-0.3 \mathrm{~V}$ and $+0.1 \mathrm{~V}$. The double layer charging current at $0.0 \mathrm{~V}$ was used to calculate $C_{\mathrm{dl}}$, which was independent of scan rate. Electrochemical measurements were also utilized to determine the surface concentration $(\Gamma)$ of each adlayer by integrating the charge under the one-electron desorption wave for the thiolate-based coating and accounting for the roughness factor of TSG. ${ }^{8}$ Desorption voltammograms were collected by scanning cathodically from $0.0 \mathrm{~V}$ at $100 \mathrm{mV} \mathrm{s}^{-1}$.

\section{Electric Force Microscopy (EFM)}

Data were collected with a Nanoscope 3A Multimode AFM, equipped with a signal access module for external control of $V_{\text {tip. }} \Delta \Phi$ was measured while changing $V_{\text {tip }}$ at a tipsample separation of $100 \mathrm{~nm}$, and amplitude for the tip oscillation of $\sim 15 \mathrm{~nm}$. Cantilevers were purchased from MikroMasch (resonance frequency $265-400 \mathrm{kHz}$, force constant $20-75 \mathrm{~N} / \mathrm{m}$ ). These tips are coated with a $20-\mathrm{nm} \mathrm{Cr}$ film and then a 20-nm Au film, which resulted in a tip radius of $\sim 50 \mathrm{~nm}$. The sample compartment was purged with argon for 40 min prior to imaging, which was maintained for the duration of the experiments.

\section{$\underline{\text { Results }}$}

\section{Molecular Modeling}

Figure 1 depicts the molecular model of benzyl mercaptan. In this visualization, the gold surface would be positioned on the left side of the image, aligned normal to the H-S bond. This architecture results in the aromatic ring aligned close to the surface normal, with the $\mathrm{H}-\mathrm{S}$ bond then utilized as an internal marker for adlayer orientation. 


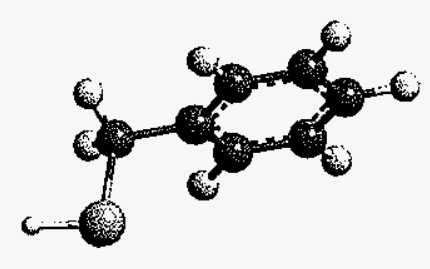

Figure 1: Molecular model of the energy-minimized benzyl mercaptan molecule. The orientation and confirmation of all the $p$-substituted benzyl mercaptans were consistent with this depiction.

The molecular coordinates of these molecules, after minimization, were employed to calculate the thickness of the adlayer. The thickness is given as the distance from the sulfur to the substituent $(\mathrm{X})$ in the direction parallel to the $\mathrm{H}-\mathrm{S}$ bond; it neglects the length of the gold-sulfur bond. These results are presented in Figure 2A, and are consistent with expectations. That is, the monolayer with $\mathrm{H}$ in the para position has the lowest thickness, whereas the $t$-butyl containing adlayer has the largest thickness. In all cases, the results yield an adlayer in which the plane of the aromatic ring is tilted $11.6^{\circ} \pm 0.5^{\circ}$ from the surface normal. These data are in general agreement with thicknesses determined by optical ellipsometry ${ }^{9}$ and with the ring orientations found by molecular dynamics calculations ${ }^{10}$ in similar systems. The molecular coordinates were also utilized to calculate both the closest

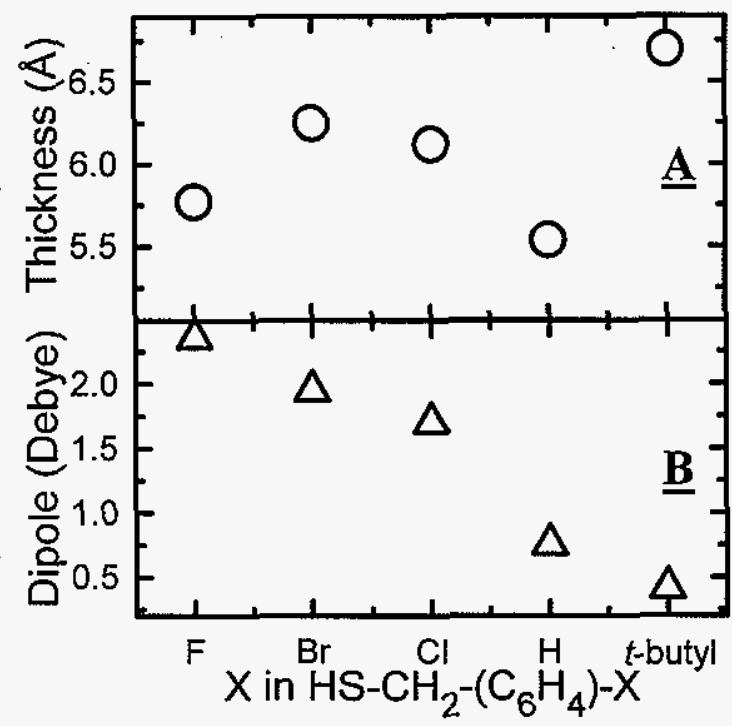

Figure 2: (A) Calculated adlayer thickness $(\mathrm{O})$ and $(\mathrm{B})$ dipole moment $(\Delta)$ with respect to the surface normal. 
packed area per molecule $(A)$ and $\Gamma$ for each of these adlayers, and are listed in Table 1 . This analysis utilized the van der Waals radius of each atom, coupled with the projected ring orientation. The trend in molecular area follows intuition, such that the larger substituents (e.g., $t$-butyl) exhibit a larger surface area.

Table 1: Summary of theoretical and experimental values related to EFM contrast mechanism for a series of para-substituted benzyl thiolate monolayers on gold.

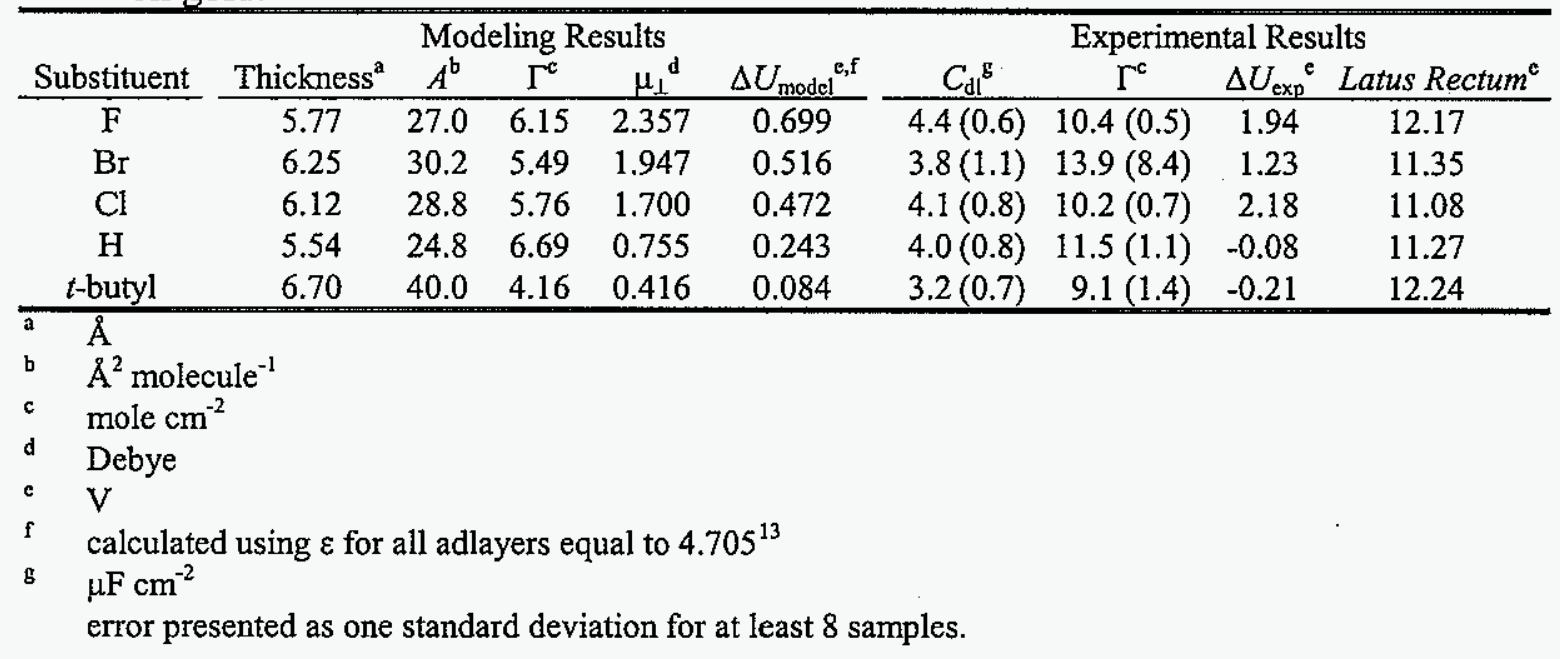

The most important results from these analyses are the dipole moments $\left(\mu_{\perp}\right)$.

Although the MOPAC software modeled the thiol precursors, the calculated dipole moment should be directly proportional to that of the corresponding adlayer because of the strong similarity in the thiolate linkages to the gold substrate. Data from X-ray photoelectron spectroscopy support this argument in that the positions of the $S(2 p)$ couplet are virtually identical for similar systems. ${ }^{11}$ This claim also applies to contributions from image dipoles created in the substrate by the adlayers. The results of the dipole moment calculations are shown in Figure 2B and tabulated in Table 1. As evident, the fluorinated adlayer has the largest dipole moment, followed by the brominated, chlorinated, and hydrogenated adlayers; the $t$-butyl derivative has the lowest value. This trend follows insights based on electronegativity and charge separation considerations. It suggests that the fluorinated 
monolayer should induce the largest modulation of the voltage gradient between the tip and substrate.

The modeled data are utilized to calculate the theoretically predicted surface potential ( $\Delta U_{\text {model }}$ ) using Equation $3,{ }^{12}$ where $A$ is in units of $\mathrm{m}^{2}, \varepsilon$ is the estimated relative permittivity of the monolayer $(4.705),{ }^{13} \varepsilon_{0}$ is the permittivity of free space $\left(8.854 \times 10^{-12} \mathrm{C} \mathrm{m}\right), \mu_{1}$ is the dipole moment perpendicular to the surface (Debye), and $\Delta U$ is the expected change in surface potential with respect to the unmodified surface $(V)$. The results of these calculations are presented in Table 1, and follow the trend based on the values of $\mu_{\perp}$.

$$
\Delta U_{\text {model }}=3.34 \times 10^{-30} \frac{\mu_{1}}{\varepsilon \varepsilon_{0} A}
$$

\section{Electrochemistry}

The $C_{\mathrm{dl}}$ and $\Gamma$ of the adlayers were determined by electrochemistry. These results are presented in Table 1. As is evident, the values of $C_{\mathrm{dl}}$ are much lower than that of an uncoated gold electrode $\left(\sim 20 \mu \mathrm{F} \mathrm{cm}^{-2}\right){ }^{8}$ Although nearly masked by the uncertainty of these measurements, the correlation of the capacitance values and substituent identity is close to that observed for the calculated dipole moments and surface potentials. That is, the $C_{\mathrm{dl}}$ for the adlayer with the $t$-butyl and hydrogen substituents are lower than those with the halogens in the para position. There is, however, one notable difference: the $C_{\mathrm{dl}}$-value for the brominated adlayer is marginally less than that for the two remaining halogenated systems as well as that for the hydrogenated adlayer.

The values of $\Gamma$ follow the same general trend as the theoretical values from the modeling calculations, with one exception: the brominated adlayer resulted in a higher coverage than the other adlayers. This discrepancy potentially stems from two factors: 1) the presence of faradic process upon adlayer desorption, and/or 2) incomplete electrochemical 
background subtraction. Experiments are being designed to examine the possibilities for the discrepancies in the $C_{\mathrm{dl}}$ and $\Gamma$ findings.

\section{EFM}

Values of $\Delta \Phi$ were measured as a function of $V_{\text {tip }}$ at a constant lift height $(100 \mathrm{~nm})$. These results are plotted in Figure 3, and summarized in Table 1 in terms of the experimentally determined surface potential $\left(\Delta U_{\text {exp }}\right)$ and the shape of the resulting profile (i.e., the length of the $L R$ ). In each case, the dependence of $\Delta \Phi$ on $V_{\text {tip }}$ exhibits a parabolic shape as predicted by Equation 2. The plots also show that the $t$-butyl monolayer has the most negative value of $\Delta U_{\text {exp }}$, whereas the Cl-terminated monolayer has the most positive value.

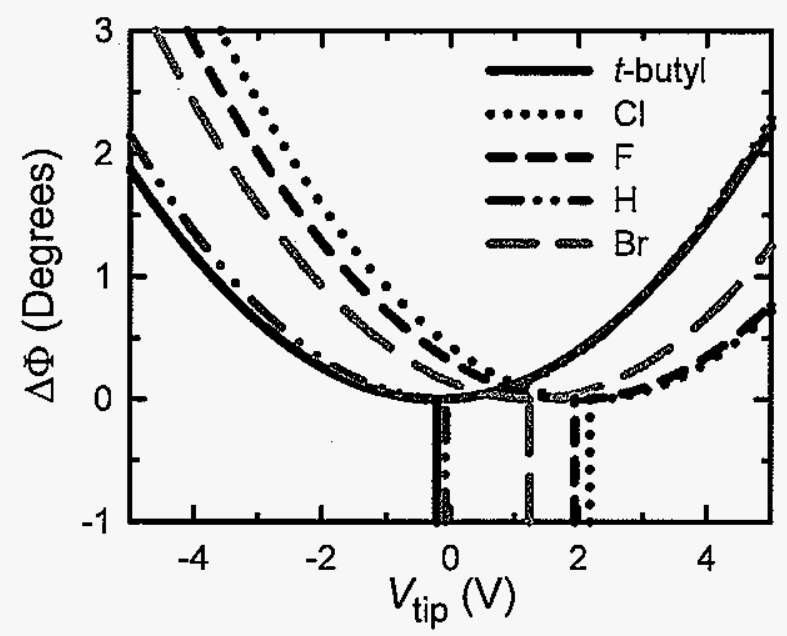

Figure 3: Experimental $\Delta \Phi$ vs. $V_{\text {tip }}$ with substrates of five different para-substituted benzyl mercaptan monolayers.

\section{Discussion}

The curves in Figure 3 yield several important observations. First, the $V_{\text {tip }}$ at the parabolic vertex $\left(\Delta U_{\text {exp }}\right.$ ) of the plot for each adlayer is clearly different. Moreover, the trend in $\Delta U_{\exp }(t$-butyl $<\mathrm{H}<\mathrm{Br}<\mathrm{F}<\mathrm{Cl})$ closely, but not fully, matches with that for $\Delta U_{\text {model }}$ ( $t$-butyl $<\mathrm{H}<\mathrm{Cl}<\mathrm{Br}<\mathrm{F}$ ). This agreement supports the possibility that the dipole moment is the major contributor to image contrast in EFM. 
However, the lack of complete agreement points to several issues that require further investigation. From the prospective of modeling, the key limitation in the calculation of $\Delta U_{\text {model }}$ rests with the values for $\varepsilon$. Our analysis is presently limited by the lack of literature data for all the test systems. While $\varepsilon$ for benzyl mercaptan has been reported, ${ }^{13}$ we have not been able to locate values for the remaining precursors. The literature for structurally similar compounds (e.g., benzenes and para-substituted toluenes) indicate that the value of $\varepsilon$ may differ by up to $60 \%$, indicating that only a qualitative comparison between $\Delta U_{\text {model }}$ and $\Delta U_{\text {exp }}$ can be reliably made at this time.

There are also several important refinements to address in the experimental area. First, the $\Delta \Phi$ curves of Figure 3 have a parabolic shape that is dependant upon the capacitance of the media between the tip and substrate. However, the shapes, as judged from the $L R$ values, do not track with the values of $C_{\mathrm{d} \text { l. }}$. We suspect that this situation reflects the presence of adventitious impurities adsorbed on the tip and sample. To address this issue, we are examining issues related to possible surface contaminates on the adlayer and tip as well as approaches to improve determination of $C_{\mathrm{d} l}$ and $\Gamma$, part of which will address methods to more reproducibly prepare the adlayers.

\section{Conclusion}

This paper has presented preliminary results that tested the effect of electronegative substituents on benzyl mercaptan-based monolayers assembled on gold surfaces. Electric force microscopy was utilized to interrogate the surface potential and capacitance of these adlayers. A theoretical model was developed to predict the expected trends in the EFM response. While preliminary, the results show that the dipole moment of the adlayer plays an important role in the contrast mechanism. Experiments to extend these first findings are planned. 


\section{Acknowledgements}

This work was supported by the Basic Energy Sciences program of the U.S.

Department of Energy. G.A.E. gratefully acknowledges a Conoco-Phillips graduate research fellowship. Valuable discussions with M. Gordon, I. Adamovic, and D. Zorn are also acknowledged. The Ames Laboratory is operated by Iowa State University for the U.S. Department of Energy under Contract W-7405-ENG-82.

\section{$\underline{\text { References }}$}

(1) Takano, H.; Kenseth, J. R.; Wong, S.-S.; O'Brien, J. C.; Porter, M. D. Chem. Rev. 1999, 99, 2845-90.

(2) Fujihira, M. Annu. Rev. Mater. Sci. 1999, 29, 353-80.

(3) a) Takano, H.; Porter, M. D. J. Am. Chem. Soc. 2001, 123, 8412-3; b) Takano, H.; Wong, S.-S.; Harnisch, J. A.; Porter, M. D. Langmuir 2000, 16, 5231-3.

(4) Sarid, D. Scanning force microscopy: with applications to electric, magnetic, and atomic forces; Oxford University Press: New York, 1994.

(5) Stamou, D.; Gourdon, D.; Liley, M.; Burnham, N. A.; Kulik, A.; Vogel, H.; Duschl, C. Langmuir 1997, 13, 2425-8.

(6) Wong, S.-S.; Porter, M. D. J. Electroanal. Chem. 2000, 485, 135-43.

(7) Chem3D, Ultra 8.0, CambridgeSoft: Cambridge MA, 2004.

(8) Widrig, C. A.; Chung, C.; Porter, M. D. J. Electroanal. Chem. 1991, 310, 335-59.

(9) Jung, H. H.; Won, Y. D.; Shin, S.; Kim, K. Langmuir 1999, 15, 1147-54.

(10) a) Tao, Y.-T.; Wu, C.-C.; Eu, J.-Y.; Lin, W.-L. Langmuir 1997, 13, 4018-23; b) Howell, S.; Kuila, D.; Kasibhatla, B.; Kubiak, C. P.; Janes, D.; Reifenberger, R. Langmuir 2002, 18, 5120-5.

(11) Zhong, C.-J.; Brush, R. C.; Anderegg, J.; Porter, M. D. Langmuir 1999, 15, 518-25.

(12) Evans, S. D.; Urankar, E.; Ulman, A.; Ferris, N. J. Am. Chem. Soc. 1991, 113, 412131 .

(13) Lide, D. R., Ed. Handbook of Chemistry and Physics, 76 ed.; CRC Press: Boca Raton, 1995. 


\section{CHAPTER 6: SUMMARY AND PROSPECTUS}

\section{General Summary}

This dissertation presents original work exploring the use of self-assembled monolayers in current research areas. Specifically, the underlying molecular properties of the components and the 3-D structure of the adlayer are related to the properties of the underlying surface.

The first research chapter (Chapter 2) contains the presentation of the first reported discovery of a dependence on the odd-even character of $n$-alkanethiolate monolayers on gold for the heterogeneous electron-transfer kinetics of solution-based redox couples. The physical foundations of this observation are discussed. Suggested explanations hinge on the disparity in properties between odd- and even-chain length adlayers, including: the oscillation in thickness, ${ }^{1}$ a difference in electronic coupling factors, ${ }^{2-4}$ or structural variations at or immediately surrounding defects.

The third Chapter includes a molecular modeling investigation of adlayer structure, which includes the molecular dipole moment and thickness. Comparison of the modeling results with experimental contact angle and ellipsometric thickness data suggest that modeling presents a very promising technique for predicting interfacial properties. This Chapter also contains theoretical and experimental evidence for odd-even oscillations in both wettability and thickness of $n$-alkanethiolate monolayers on gold.

Chapter 4 details investigations into the controlled mass transport of thiol precursor molecules to the gold surface. It discusses the importance of the deposition conditions on the reproducibility of the adlayer structure and resulting interfacial properties of the system. A theoretical model is derived that relates substrate/vessel geometry, deposition solution concentration, and thiol mass transport to the surface. The model is then utilized as a 
platform to assess the adlayer structure dependence on deposition parameters. This work unequivocally states impingement number and rate, as controlled by immersion time, thiol concentration, and deposition geometry are extremely important variables in self-assembly. Future work discussing the evolution of adlayer structure/function as deposition conditions are varied will use this model as a stepping-stone in data analysis and discussion.

Chapter 5 completes the research portion of the dissertation with an investigation into the theoretical basis for the contrast mechanism in electric force microscopy (EFM) of monolayer samples, using the model system of benzyl mercaptan-derived adlayers. Again, molecular modeling is utilized to calculate the expected dipole moment of adlayer, which was then translated to an estimated shift in surface potential. Each adlayer was interrogated by EFM, with a simultaneous measurement of the layer capacitance and surface potential. While preliminary, the results presented in this Chapter show that the dipole moment of the adlayer plays an important role in the EFM contrast mechanism. In addition, we have shown that EFM is a facile way to investigate the capacitance and surface potential of a variety of sample surfaces.

\section{Prospectus}

The reproducible control of interfacial properties afforded by self-assembled monolayers has facilitated groundbreaking research. In order to allow continuing advancement, a fundamental understanding of these interfaces is required. For instance, the root causes of the odd-even oscillation in heterogeneous electron-transfer rate (Chapter 2) at these electrodes may allow the ability to engineer and assemble selective interfaces for a wide variety of uses, including chemical sensors. Therefore, investigations into the controlling factors of the interfacial properties of self-assembled monolayers are ongoing.

In this light, a renewed interest in the reproducible preparation of these interfaces will require a further exploration of the kinetics and individual stages of self-assembly. The 
mass-transfer models derived in Chapter 4 will be utilized, and possibly extended to consider deposition temperature, solvent viscosity, and a better approximation of the sticking coefficient of absorption. The diffusion models also beg the question of addition masstransport methods including convection. For example, the use of methodologies borrowed from the electrochemical rotating disk electrode literature, in which the delivery of molecules is a direct result of solution convection controlled by the rotation speed of the electrode.

Researchers also strive to predict properties of specific interfaces before assembly. Chapter 3 presents a way to calculate the expected dipole and thickness of the adlayer. These models can be extended to other systems quickly if the packing structure and adlayer orientation is known. We can therefore predict the trends in contact angle values for substituents of the methylene chain, such as the methyl group functionalization.

Chapter 5 delves into the description of the contrast mechanism for EFM. Although this technique is most useful for imaging buried interfaces, ${ }^{5,6}$ the developed model does not account for the polymer layer. An envisioned extension includes modeling the buried system with a 3-D modeling package that will accommodate electrical fields, multilayer samples, and a more dynamic simulation of the forces encountered by the oscillating tip.

\section{$\underline{\text { References }}$}

(1) Smalley, J. F.; Feldberg, S. W.; Chidsey, C. E. D.; Linford, M. R.; Newton, M. D.; Liu, Y.-P. J. Phys. Chem. 1995, 99, 13141-9.

(2) Cheng, J.; Miller, C. J. J. Phys. Chem. B 1997, 101, 1058-62.

(3) Hsu, C.-P. J. Electroanal. Chem. 1997, 438, 27-35.

(4) Hsu, C.-P.; Marcus, R. A. J. Chem. Phys. 1997, 106, 584-98.

(5) Takano, H.; Porter, M. D. J. Am. Chem. Soc. 2001, 123, 8412-3.

(6) Takano, H.; Wong, S.-S.; Harnisch, J. A.; Porter, M. D. Langmuir 2000, 16, 5231-3. 


\section{ACKNOWLEDGEMENTS}

Funding for this work was provided by the Basic Energy Sciences program of the U.S. Department of Energy. I also gratefully acknowledge a Conoco-Phillips graduate research fellowship.

The author gratefully appreciates the guidance of his advisor, Prof. Marc D. Porter, and valuable discussions with I. Adamovic, and D. Zorn as well as Professors A. Hillier and M. Gordon at Iowa State University. Past and current members of Marc Porter's group have also been supportive throughout the completion of this work. Special thanks are given to J. Driskell and A. Bergren for many helpful discussions.

This work was performed at Ames Laboratory under Contract No. W-7405-ENG-82 with the U.S. Department of Energy. The United States government has assigned the DOE Report number IS-T 2042 to this dissertation. 